University of Tennessee Health Science Center UTHSC Digital Commons

$12-2006$

\title{
Biomechanical Testing of Two-Level Cervical Disc Arthroplasty
}

Laura J. Gilmour

University of Tennessee Health Science Center

Follow this and additional works at: https://dc.uthsc.edu/dissertations

Part of the Surgical Procedures, Operative Commons

\section{Recommended Citation}

Gilmour, Laura J. , "Biomechanical Testing of Two-Level Cervical Disc Arthroplasty" (2006). Theses and Dissertations (ETD). Paper 103. http://dx.doi.org/10.21007/etd.cghs.2006.0111.

This Thesis is brought to you for free and open access by the College of Graduate Health Sciences at UTHSC Digital Commons. It has been accepted for inclusion in Theses and Dissertations (ETD) by an authorized administrator of UTHSC Digital Commons. For more information, please contact jwelch30@uthsc.edu. 


\title{
Biomechanical Testing of Two-Level Cervical Disc Arthroplasty
}

\begin{abstract}
In vitro biomechanical studies comparing two-level cervical disc arthroplasty with two-level fusion were completed using an established cadaveric cervical spine model. Three conditions were tested: noninstrumented, instrumented with two-level fusion (C5-C6 and C6-C7), and instrumented with two-level arthroplasty (C5-C6, C6-C7) using the Prestige Low-Profile (Medtronic Sofamor Danek, Memphis TN) or ProDisc-C (Synthes Spine, West Chester PA) prosthetic disc. Specimens were tested non-destructively in physiologic flexion-extension, lateral bending, and axial rotation to an end-limit load of 3-Nm or 450 rotation. Rotations at the superior, implanted, and inferior motion segment units (MSU) of the instrumented conditions were normalized to the non-instrumented condition and analyzed using one-way ANOVA and Student-Newman-Keuls test $(p<0.05)$. Two-level fusion significantly decreased motion at the implanted levels compared to the harvested condition and significantly increased motion at adjacent levels. The motion response at the implanted levels of the two-level Prestige did not significantly differ from the harvested condition, except in flexion, extension and combined flexion plus extension. The ProDisc-C prosthesis showed a similar motion response to the harvested condition at the implanted levels except in flexion, and left axial rotation. Upon direct comparison of the two devices, the Prestige-LP had significantly greater motion in extension and the ProDisc-C had significantly greater motion in axial rotation relative to the harvested condition. Differences in motion between the devices were due to differences within the mechanical designs. The Prestige-LP is a more mobile device in the anteriorposterior plane, which explains the increase in motion in extension, while the ProDisc-C prosthesis has a more constrained design. Overall, two-level disc arthroplasty maintained motion at the implanted levels and did not show a significant difference at adjacent levels, indicating two-level arthroplasty may be a viable alternative treatment for multi-level degenerative cervical disc disease.
\end{abstract}

\section{Document Type}

Thesis

Degree Name

Master of Science (MS)

Program

Biomedical Engineering

Research Advisor

Denis J. DiAngelo, Ph.D.

\section{Keywords}

cervical spine, two-level disc arthroplasty, two-level fusion, biomechanical testing

\section{Subject Categories}

Analytical, Diagnostic and Therapeutic Techniques and Equipment | Medicine and Health Sciences |

Surgical Procedures, Operative 


\title{
BIOMECHANICAL TESTING OF TWO-LEVEL CERVICAL DISC ARTHROPLASTY
}

\author{
A Thesis \\ Presented for \\ The Graduate Studies Council \\ The University of Tennessee \\ Health Science Center
}

\author{
In Partial Fulfillment \\ Of the Requirements for the Degree \\ Master of Science \\ In The Joint Graduate Program in Biomedical Engineering \\ From The University of Tennessee \\ And \\ The University of Memphis
}

By

Laura J. Gilmour

December, 2006 


\section{Dedication}

This thesis is dedicated to my parents,

Mr. Robert C. Gilmour and Mrs. Jan T. Gilmour

for their love and support throughout my education and personal endeavors. 


\section{Acknowledgements}

My gratitude is extended to my advisor Dr. Denis DiAngelo for the opportunity to work and learn in his laboratory, my committee Dr. Richard Kasser and Dr. Michael Yen, my fellow lab members Brian Morrow, John Schwab, and Dr. Brian Kelly for all of their help and guidance, the Medical Education Research Institute for their continued generosity in the use of their

facilities, and neurosurgeons Dr. Kevin Foley, Dr. Kelly Kiehm, and Dr. John German for surgical expertise in implantation of the devices. 


\begin{abstract}
In vitro biomechanical studies comparing two-level cervical disc arthroplasty with twolevel fusion were completed using an established cadaveric cervical spine model. Three conditions were tested: non-instrumented, instrumented with two-level fusion (C5-C6 and C6C7), and instrumented with two-level arthroplasty (C5-C6, C6-C7) using the Prestige LowProfile (Medtronic Sofamor Danek, Memphis TN) or ProDisc-C (Synthes Spine, West Chester PA) prosthetic disc. Specimens were tested non-destructively in physiologic flexion-extension, lateral bending, and axial rotation to an end-limit load of $3-\mathrm{Nm}$ or $45^{\circ}$ rotation. Rotations at the superior, implanted, and inferior motion segment units (MSU) of the instrumented conditions were normalized to the non-instrumented condition and analyzed using one-way ANOVA and Student-Newman-Keuls test $(\mathrm{p}<0.05)$. Two-level fusion significantly decreased motion at the implanted levels compared to the harvested condition and significantly increased motion at adjacent levels. The motion response at the implanted levels of the two-level Prestige did not significantly differ from the harvested condition, except in flexion, extension and combined flexion plus extension. The ProDisc-C prosthesis showed a similar motion response to the harvested condition at the implanted levels except in flexion, and left axial rotation. Upon direct comparison of the two devices, the Prestige-LP had significantly greater motion in extension and the ProDisc-C had significantly greater motion in axial rotation relative to the harvested condition. Differences in motion between the devices were due to differences within the mechanical designs. The Prestige-LP is a more mobile device in the anterior-posterior plane, which explains the increase in motion in extension, while the ProDisc-C prosthesis has a more constrained design. Overall, two-level disc arthroplasty maintained motion at the implanted
\end{abstract}


levels and did not show a significant difference at adjacent levels, indicating two-level arthroplasty may be a viable alternative treatment for multi-level degenerative cervical disc disease. 


\section{Table of Contents}

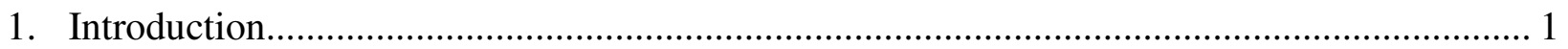

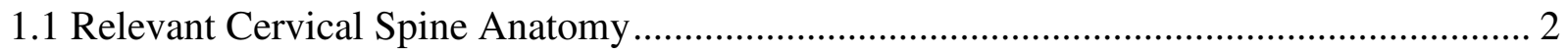

1.2 Arthrodesis Versus Arthroplasty................................................................................. 5

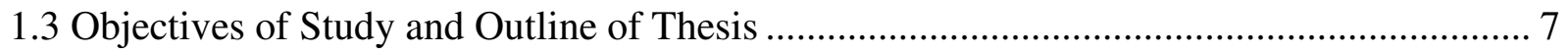

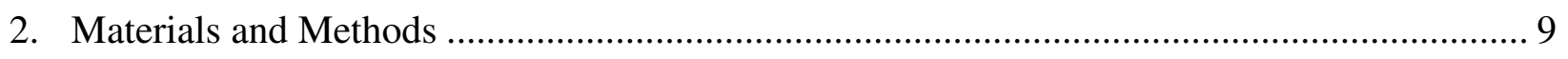

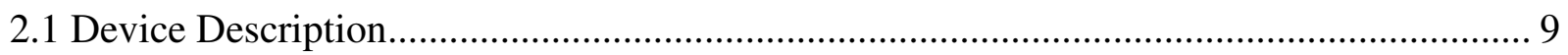

2.1.1 Prestige Low Profile Disc Prosthesis ............................................................................ 9

2.1.2 ProDisc-C Disc Prosthesis ..................................................................................... 10

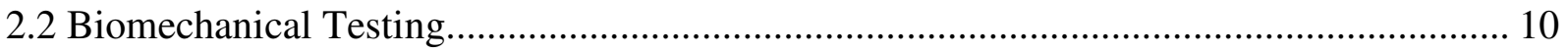

2.3 Data Management and Statistical Analysis................................................................. 14

3. Prestige-LP Cervical Arthroplasty Versus Fusion at Two Levels: What Are the

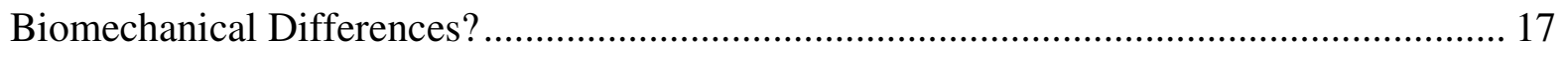

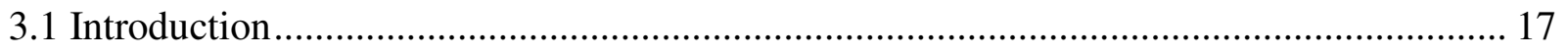

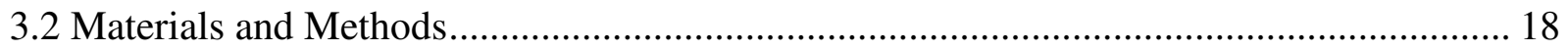

3.2.1 Specimen Preparation and Spinal Conditions......................................................... 18

3.2.2 Non-Destructive Testing Protocol ....................................................................... 20

3.2.3 Data Management and Statistical Analysis................................................................. 21

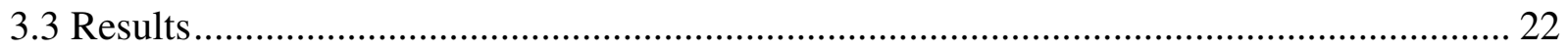

3.3.1 Normalized Flexibility ……………………………............................................... 22

3.3.2 Normalized Motion ............................................................................................. 22

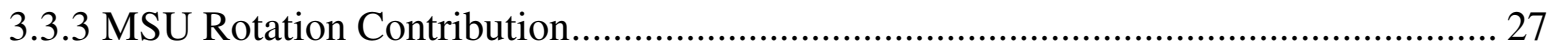




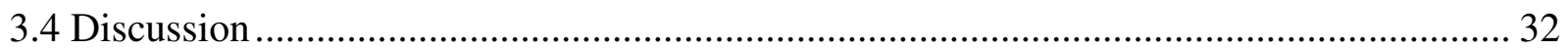

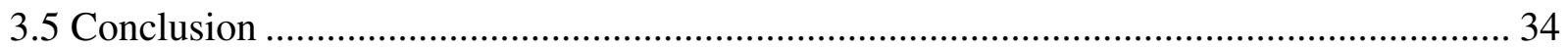

4. Biomechanical Comparison of Two-Level Arthroplasty Using the ProDisc-C with Two-

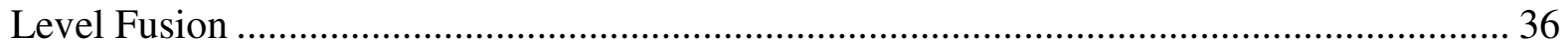

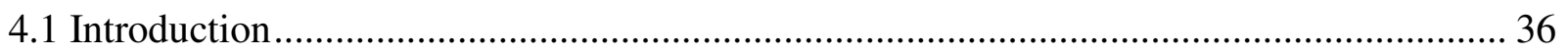

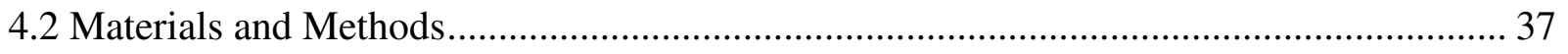

4.2.1 Specimen Preparation and Spinal Instrumentation .................................................. 37

4.2.2 Non-Destructive Biomechanical Testing Protocol .................................................... 38

4.2.3 Data Management and Statistical Analysis.................................................................. 40

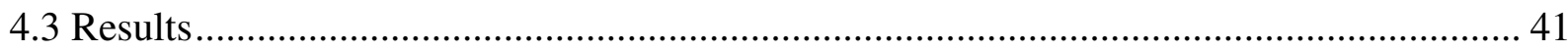

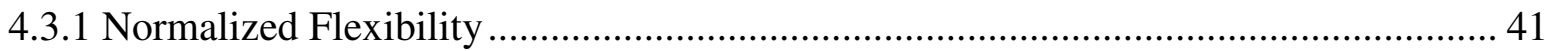

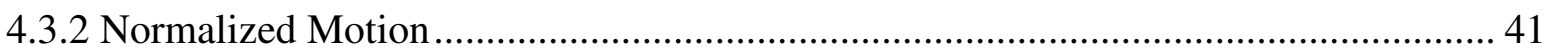

4.3.3 Changes in MSU Contribution..................................................................................... 45

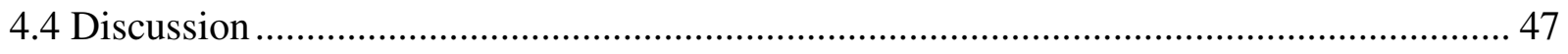

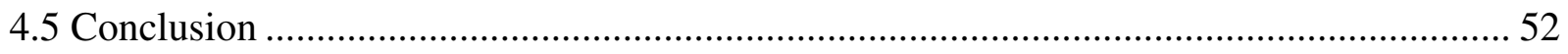

5. Multi-Level Disc Arthroplasty: A Comparison of Two Prosthetic Disc Devices .................. 55

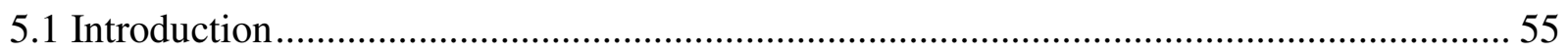

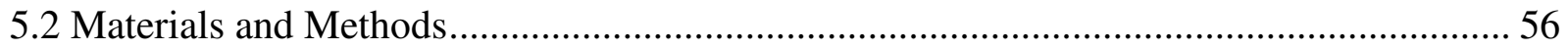

5.2.1 Specimen Preparation and Spinal Conditions ............................................................. 56

5.2.2 Non-Destructive Biomechanical Testing ............................................................... 59

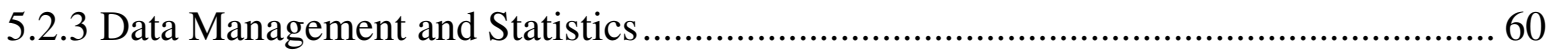

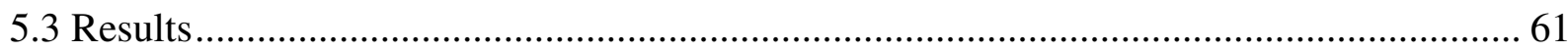

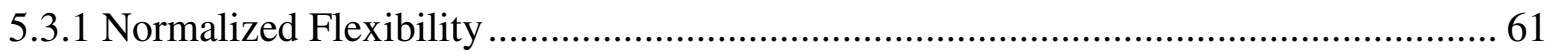




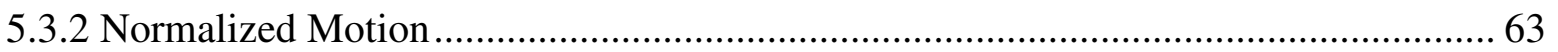

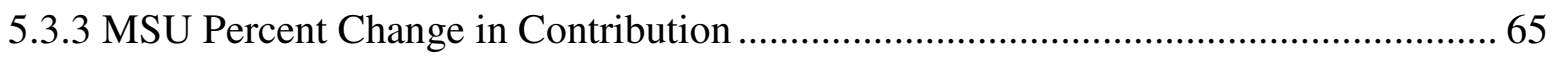

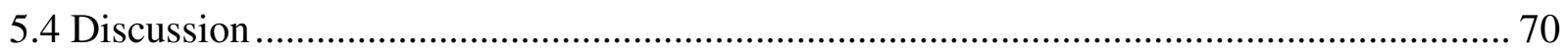

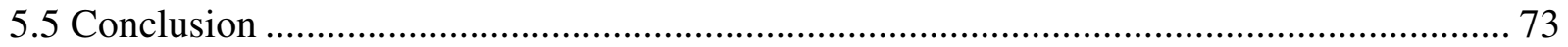

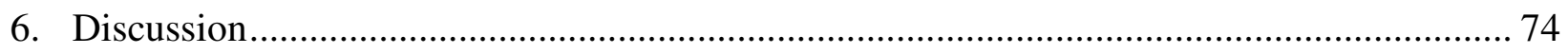

6.1 Summary of Two-Level Prestige-LP Versus Two-Level Fusion ...................................... 76

6.2 Summary of Two-Level ProDisc-C Versus Two-Level Fusion ........................................... 77

6.3 Summary of Two-Level Prestige-LP Versus Two-Level ProDisc-C ................................. 77

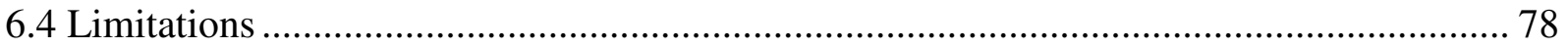

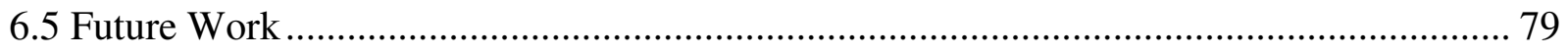

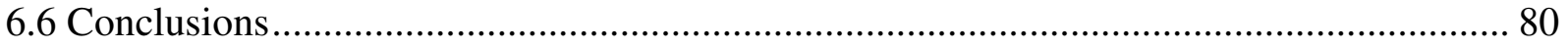

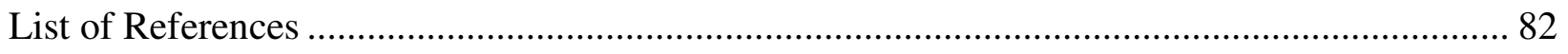

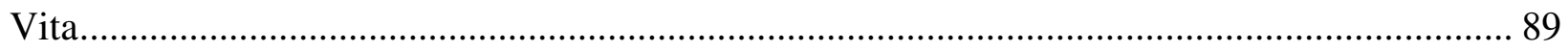




\section{List of Tables}

Table 3-1: Global Rotation Versus Applied Moment Data. ..................................................... 23

Table 3-2: Motion at Treated Level.............................................................................. 25

Table 4-1: Global Rotation Versus Applied Moment Data for ProDisc-C................................ 42

Table 4-2: Normalized Flexibility for ProDisc-C Study................................................... 42

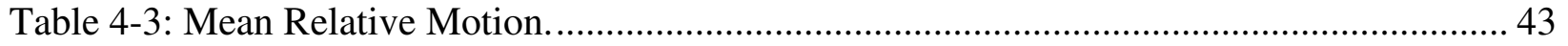

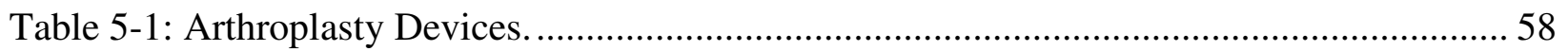




\section{List of Figures}

Figure 1-1: Typical Cervical Vertebra........................................................................... 4

Figure 2-1: Custom Designed Non-Destructive Biomechanical Testing Machine. ................. 12

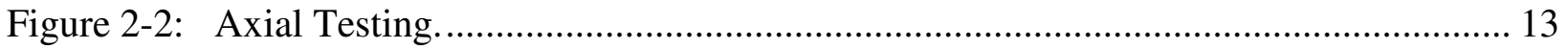

Figure 2-3: MSU Percent Change in Contribution Calculation.............................................. 16

Figure 3-1: Prestige Low-Profile Disc Prosthesis.................................................................. 19

Figure 3-2: Test Prepared Specimens. ............................................................................ 19

Figure 3-3: Biomechanical Testing Apparatus. ............................................................. 20

Figure 3-4: Prestige-LP Normalized Flexibility for All Loading Cases.................................. 24

Figure 3-5: Prestige-LP Normalized Motion in Flexion and Extension................................ 26

Figure 3-6: Prestige-LP Normalized Motion in Lateral Bending. ....................................... 26

Figure 3-7: Prestige-LP Normalized Motion in Axial Rotation. ........................................... 27

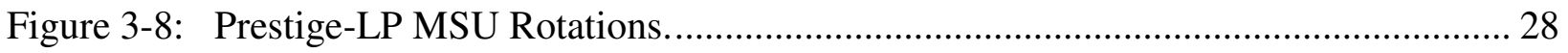

Figure 3-9: Prestige-LP MSU Percent Change in Contribution in Flexion and Extension. ....... 29

Figure 3-10: Prestige-LP MSU Percent Change in Contribution in Lateral Bending. ................ 30

Figure 3-11: Prestige-LP MSU Percent Change in Contribution in Axial Rotation................... 31

Figure 4-1: ProDisc-C Disc Prosthesis....................................................................... 39

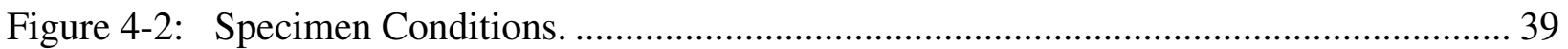

Figure 4-3: ProDisc-C Normalized Motion for Flexion and Extension. ................................ 44

Figure 4-4: ProDisc-C Normalized Motion for Lateral Bending ........................................ 44

Figure 4-5: ProDisc-C Normalized Motion for Axial Rotation.......................................... 45

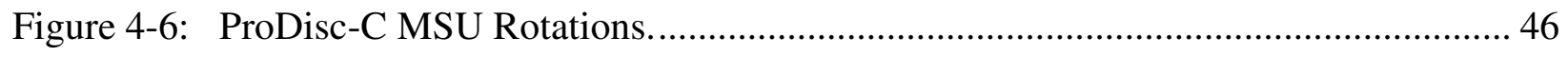




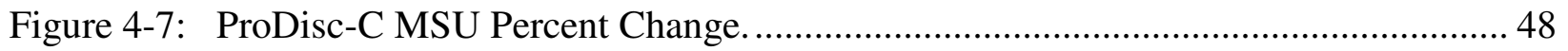

Figure 4-8: ProDisc-C MSU Percent Change in Lateral Bending......................................... 49

Figure 4-9: ProDisc-C MSU Percent Change in Axial Rotation. .......................................... 50

Figure 4-10: Normalized Motion Comparing One-Level and Two-Level ProDisc-C. ............... 53

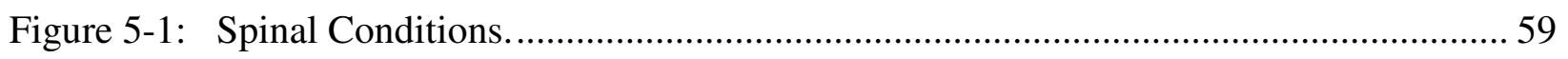

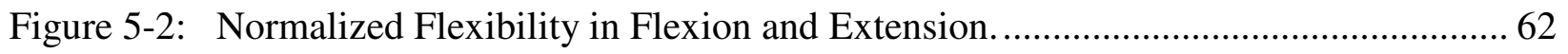

Figure 5-3: Normalized Flexibility in Lateral Bending. ............................................... 62

Figure 5-4: Normalized Flexibility in Axial Rotation.. .................................................... 63

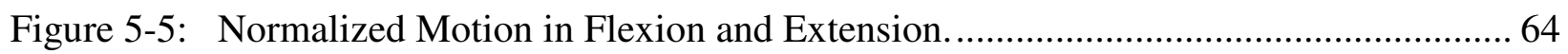

Figure 5-6: Normalized Motion in Lateral Bending. ........................................................ 64

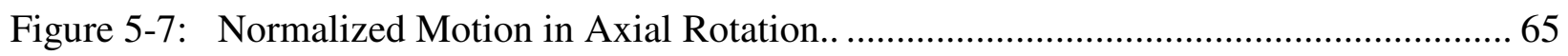

Figure 5-8: Percent Change Contribution for MSU Rotations. ............................................ 66

Figure 5-9: Lateral Bending Percent Change Contribution for MSU Rotations. ..................... 67

Figure 5-10: Axial Rotation Percent Change Contribution for MSU Rotations......................... 68 


\section{Introduction}

Cervical disc arthroplasty is currently an investigational procedure that serves as an alternative to anterior cervical discectomy and fusion (ACDF) for treating degenerative disc diseases. Many spinal arthroplasty devices have been clinically analyzed in the United States in both the lumbar and cervical spine since 2000 under the Food and Drug Administration (FDA) investigational device exemption process. ${ }^{6,25}$ The first lumbar disc arthroplasty device was approved in $2004,{ }^{6}$ and a cervical counterpart is expected to follow shortly. The biomechanics of two investigational cervical disc prostheses implanted at cervical levels C5-C6 and C6-C7 in a cadaver model was determine and compared against two-level fusion at these same treated levels.

The goal of spinal arthroplasty is to restore motion at the effected levels rather then eliminate motion, as occurs with fusion surgery. Theoretically, by maintaining motion at the diseased level, adjacent levels do not experience an unnatural increase in compensatory motion, and adjacent segment disease experienced with fusion may be minimized or avoided. Adjacent

segment disease is a reoccurrence of degenerative disc disease adjacent to the treated level. ${ }^{3,9,16 \text {, }}$ $18,22,24,27,31,36,38,41,48$ Other theoretical advantages include re-establishment of disc height and spinal alignment, decreased surgical morbidity compared to fusion, preservation of mechanical characteristics of the spine, a decreased recovery time, and elimination of postoperative immobilization or fusion instrumentation complications that occurs with plating, especially in a multi-level situation. ${ }^{3}$ These advantages make disc arthroplasty a very attractive surgical procedure for treatment of degenerative diseases of the cervical spine. 
This introduction provides a brief overview of the relevant cervical spinal anatomy, discuss the rational for disc arthroplasty as an alternative to fusion, and explain why two-level instrumentation was studied. A brief outline of the thesis concludes the introduction.

\subsection{Relevant Cervical Spine Anatomy}

The cervical spine is divided into upper $(\mathrm{C} 0-\mathrm{C} 2)$ and lower $(\mathrm{C} 3-\mathrm{C} 7)$ segments. The lower cervical spine was the subject of these biomechanical studies due to the complexity of the $\mathrm{C} 0-\mathrm{C} 1-\mathrm{C} 2$ complex, the lack of a true synovial joint and disc material in this region, and interest in the lower portion's disease mechanisms and potential treatments. In this body of research, the sub-axial cervical spine (C2-T1) was considered. The T1 vertebral body is anatomically similar to the vertebrae of the cervical spine despite its designation of a thoracic vertebra. ${ }^{1}$

When replacement for human anatomy is investigated it becomes essential to understand the anatomy. In this application it is important to understand the native disc, as well as the entire vertebral joint, recognizing the importance of motion preservation and its role as a mobile unit.

The six cervical discs, of the sub-axial cervical spine are similar in humans and closely related to discs of other spinal regions. The first cervical disc exists between C2-C3. A complete synoval joint is absent between the occiput $(\mathrm{C} 0)$ and $\mathrm{C} 1$ as well as between the $\mathrm{C} 1-\mathrm{C} 2$ motion segment unit (MSU). Anatomically, the spinal disc consists of an outer fibrous ring, the annulus fibrosus, and a semisolid central core called the nucleus pulposus. Biomechanically, spinal discs are very strong when compressed, and the transmission of a compressive force occurs without producing an extreme local concentration of stress. A posterior shift of the nucleus pulposus occurs during flexion, while an anterior shift occurs during extension. This movement pattern produces a load leveling effect. ${ }^{1,47}$ 
The vertebra shown in Figure $1-1^{10}$ is made up of the pedicles, transverse processes, superior and inferior articular processes, facet joints, spinous processes and finally the spinal canal. The pedicles are located anteriolaterally and inferiorly and connect the vertebral body to the posterior elements. The transverse processes are located posterolaterally from the pedicles, and are the insertion points for the anterior and posterior neck muscles. The superior and inferior articular processes are connected posteriorly to pedicles. The articular facet is also located here. The facet joints provide anteriorposterior translational stability and allow intervertebral motion. Facet joint orientation changes from an almost transverse oriented at C1-C2, through to a 55-60 degree angle from $\mathrm{C} 2-\mathrm{C} 3$ through $\mathrm{C} 7-\mathrm{T} 1$. The spinous processes are attached to the lateral mass through the lamina and increase in length from C3 through T1. This length increase provides greater torque required to move the head. The spinous processes are arranged in a bifid formation that allows them to nest with one another providing maximum extension without necessary decrease in spinous process length. The vertebral body pedicles, lateral masses, and lamina form the spinal canal. ${ }^{1,10,47}$

In specimen preparation for biomechanical testing (described in later sections) the ligaments were carefully preserved. This preservation was due to the fact that the ligaments are instrumental in spinal stability. Cervical ligments are divided into two groups: 1. those spanning multiple motion segment units and 2. those attached through only one motion segment unit. One of the most important multi-segment spanning ligaments is the ligamentum nuchae, which runs from the occipital protrusion to the $\mathrm{C} 7$ spinus process. This ligament prevents excess spinal flexion. The anterior longitudinal ligament (ALL) physically spans multiple motion segment units, but functions to limit motion across one. Firm attachment to each vertebral body limits the 


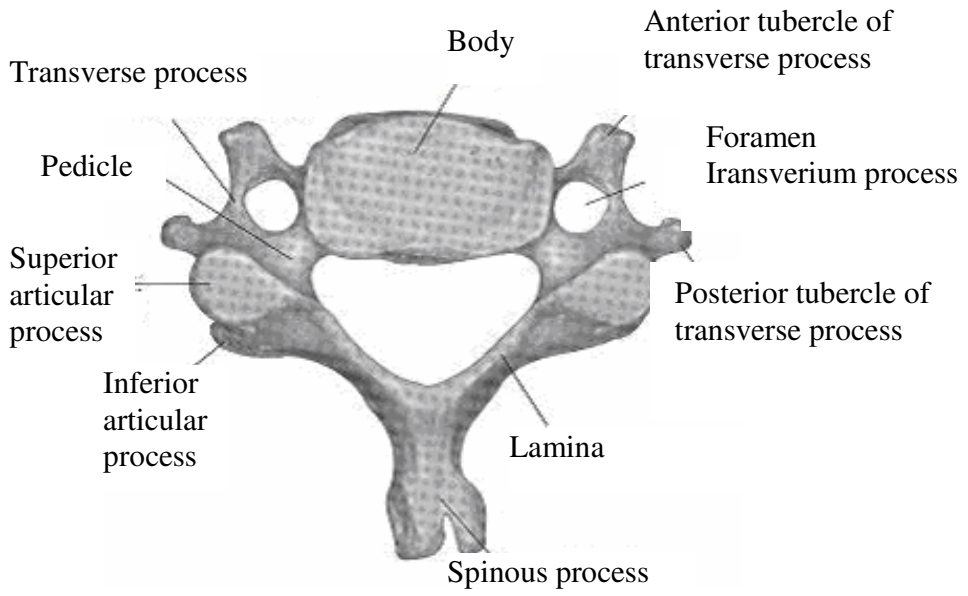

a

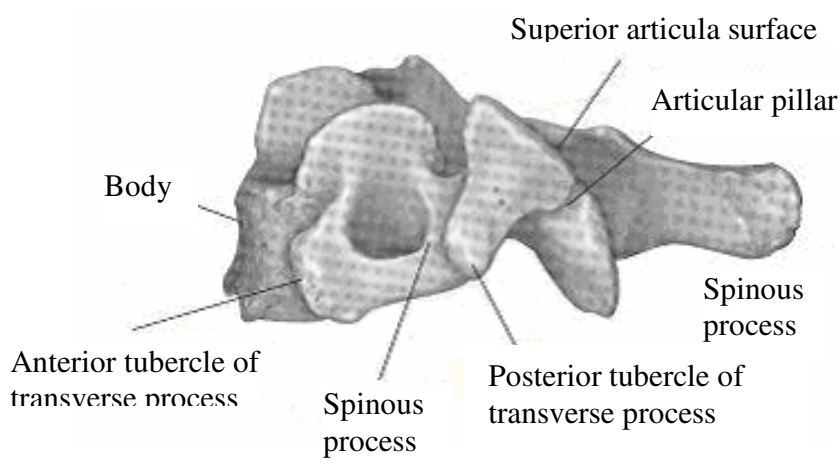

b

Figure 1-1: Typical Cervical Vertebra. (a) Caudal view. (b) Lateral view. (Used with permission from Clark C, ed. The Cervical Spine. 3rd ed. Philadelphia: Lippincott-Raven, 1998.) 
motion of that one segment but not the motion of the segment relative to other segments. The posterior longitudinal ligament (PLL) is identical to the ALL in function and located along the posterior aspect of the spinal bodies. The ligamentum flavum also functionally spans only one MSU and provides a significant stabilizing force during cervical flexion due to its high elasticity. This ligament is instrumental in returning the neck to its natural position after flexion occurs. Other ligaments that functionally span only one motion segment unit include intertransverse process ligament and facet joint capsules. 1,47

Cervical spinal muscles are instrumental in offering support and providing precise control of the head; however, due to the cadaveric nature of this study only ligament and disc behavior were studied. In the case of the cadaveric model, muscles are not active and were not included.

\subsection{Arthrodesis Versus Arthroplasty}

The current accepted standard of care for degenerative disc diseases of the cervical spine is anterior discectomy and arthrodesis or fusion of the offensive segment. Many clinical and biomechanical studies have established a significant occurrence of adjacent segment disease following ACDF. Adjacent segment disease is the development of a new radiculopathy or

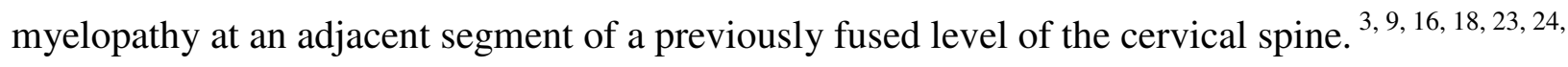
27, 31, 36, 38, 41, 48 Common adjacent level pathologies include disc herniation or degeneration, instability, stenosis, spondylosis, and facet joint arthritis. The causes of this phenomenon are not agreed upon. Hilibrand et al. ${ }^{22}$ found the levels most mobile (C5-C6 and C6-C7) were significantly more likely to be subjected to adjacent segment disease. Other studies suggest an increase in strain in the adjacent discs ${ }^{27}$, or an increase in motion at levels above or below a

fusion site ${ }^{30}$. initiate adjacent segment disease. Matsunaga et al. ${ }^{27}$ also found an increase in 
intervertebral disc strain of segments adjacent to fusion. While Azmi and Schlenk ${ }^{3}$ suggested that disc arthroplasty is an option in treating adjacent segment disease, preventing it using the same device is obviously more beneficial.

Using radiography, Goffin et al. ${ }^{18}$ found $60 \%$ of patients (15 of 25) with ACDF were diagonosed with adjacent segment disease at five to nine years follow-up. Degenerative changes of the adjacent segment occurred with patients of more then one fused level, fusion on lower cervical segments, and hyperflexion injuries. Through careful radiographic study, Hilibrand et $\mathrm{al}^{22}$ have determined the rate of adjacent segment disease to be $2.9 \%$ per year over ten years following ACDF procedures. Although they did not find a significant increase of symptomatic adjacent segment disease in patients with two-fused levels, other studies have suggested this may be the case. Hilibrand et al. ${ }^{22}$ acknowledged the incidence of adjacent segment disease is most likely at C5-C6 and C6-C7, the more mobile units of the cervical spine. Multi-level procedures usually include these two levels, which may explain the lack of adjacent segment disease found in this study for the multi-level case.

While multi-level fusion is not a new phenomenon, multi-level disc arthroplasty has been increasingly discussed in the literature. Two-level arthroplasty would be indicated for patients with a soft disc herniation and neurological symptoms at two levels who were not experiencing hypermobility or instability. Arthroplasty has been successful for patients diagnosed with radiculopathy and myelopathy, as well as acute disc herniations or degenerative spondylotic change. There have been some clinical studies in the literature on effects of multi-level disc arthroplasty, especically using the Bryan disc; ${ }^{15,19,32,43}$ however there has been no documentation that reports in vitro biomechanical analysis of two-level cervical arthroplasty. Duggal et al. ${ }^{15}$ reported on four patients who received prostheses at two-levels. Motion 
preservation at the treated levels and an increase in overall motion of the cervical spine (C2-C7) were found at 24 months postoperatively; however, these results are collective with the single level patients. Goffin et al. ${ }^{18}$ measured motion at the treated levels radiographically and found range of motion per level averaged $7.4 \pm 5.1$ degrees in two-level patients at one year

postoperatively. Pickett et al. ${ }^{33}$ found similar results to Duggal ${ }^{15}$ and determined sagittal rotation, anterior and posterior disc height, translation, and center of rotation (COR) did not significantly change following implantation. Again, the two-level patients were not reported as a separate cohort.

The purpose of cervical discectomy and arthrodesis is to decompress the nerve roots near the spinal column and restabilize the motion segment unit. Restabilization of this nature reduces the motion at the operated levels that may increase the motion at the adjacent levels as seen in biomechanical studies. $^{12,40}$ The advantage of an ideal implantable disc prosthesis is the ability to retain natural motion while replacing the tissue causing the symptoms.

\subsection{Objectives of Study and Outline of Thesis}

The objectives of this study were to first determine the biomechanical behavior of two cervical disc prostheses in a two-level cadaveric disc arthroplasty model, then compare these to the intact, control state (harvested) and the two-level fused state, and finally to compare the biomechanical performance of the two disc prostheses to one another. Chapter 2 provides an overview of the two devices studied and introduces the testing methods. Chapter 3 discusses the biomechanical response of the Prestige Low-Profile (Medtronic Sofamor Danek, Memphis TN) disc prosthesis implanted at two-levels in the cadaver cervical spine. Chapter 4 will discuss the biomechanical response of two-level disc arthroplasty using the ProDisc-C (Synthes Spine, West 
Chester PA) disc prosthesis. Chapter 5 compares the biomechanical response between two different disc prostheses in a two-level implanted situation. A discussion follows in Chapter 6, with conclusive remarks in this final chapter. 


\section{Materials and Methods}

\subsection{Device Description}

\subsubsection{Prestige Low Profile Disc Prosthesis}

The design of the Prestige Low Profile disc has been updated twice under the same name. The Prestige I was first introduced in 1998 by Medtronic Sofamor Danek. This version consisted of a metal on metal articulating surfaces. The lower component has an elliptical shape that allows $2 \mathrm{~mm}$ of translation in the anterior-posterior (A-P) plane. ${ }^{36}$ The upper component can also glide in the lower saucer to allow some rotational movement. In theory, this rolling articulation reduces friction, thus reducing wear debris. Due to the non-congruent design of the interface, the device axis of rotation in the A-P plane is affected by facet joint interaction and coupled motions of adjacent vertebrae. The current version allows $10^{\circ}$ flexion, extension or lateral bending and $2 \mathrm{~mm}$ of translation. ${ }^{35}$ The anterior plates are anatomically contoured to fit the anterior surface of the vertebrae.

The design updates to the Prestige II included a more low-profile design and additional implant sizes. Additional sizes included an AP dimension of 12 and $14 \mathrm{~mm}$ and additional heights of 6 and $8 \mathrm{~mm}$ at each AP dimension. The articulation provided with the first Prestige model was unchanged in the second design update. The device currently studied, Prestige Low Profile (LP), has an even greater low-profile design. This device does not use screws to anchor the implant the vertebral bodies, but is fixed with only a keel mechanism similar to the ProDiscC cervical disc implant. ${ }^{35}$ 


\subsubsection{ProDisc-C Disc Prosthesis}

The ProDisc-C cervical disc design is similar to the lumbar disc prosthesis of a similar name, i.e. the ProDisc-L. It is made of two chromium-cobalt endplates with a sagittal keel fixation and a fixed ultra-high molecular weight polyethylene (UHMWP) insert. Chromiumcobalt contains cobalt, chromium, and molybdenum. Molybdenum allows for decreased grain

size and the oxidation of chromium provides a resistance to corrosion. ${ }^{46}$ A plasma spray is used on the surfaces of the implant in contact with vertebral bodies to promote boney ongrowth.

This ball and socket type joint has a concave superior component and a convex inferior component with the UHMWPE inlay attached. The three piece design is intended to allow for replacement of one portion of the implant while an entire extraction is not necessary. This design provides a fixed center of rotation located at the middle of the inferior vertebral body. No independent translation is provided by this implant while coupled rotations are ${ }^{32,37}$

The ProDisc-C has six footprint sizes, with three widths and four depths, and three implant heights to ensure proper sizing is available. The footprint of the ProDisc-C is intended to cover the entire endplate of the vertebral body surrounding the affected disc. A large footprint is necessary to prevent subsidence by allowing the weight to be transferred to the stronger periphery of the endplate rather than the center. Although subsidence is rare in the cervical spine, incidences have been widely reported in lumbar disc arthroplasty. ${ }^{2}$

\subsection{Biomechanical Testing}

It is important for non-destructive biomechanical testing systems to replicate the complicated physiological motion of the cervical spine as closely as possible when evaluating spinal instrumentation such as prosthetic cervical disc devices. Previous studies conducted by 
DiAngelo et al. have shown that an inverted spinal orientation (loading applied to T1 rather then C2) testing set-up using displacement control replicates physiological motion conditions better then pure moment biomechanical testing techniques. ${ }^{13}$ The natural load of head weight on the cervical spine causes a caudally increasing moment from $\mathrm{C} 2$ through $\mathrm{C} 7$ with the greatest moment occurring at C5-C6. Thus, an eccentrically applied compressive load induces a greater moment at $\mathrm{T} 1$ than $\mathrm{C} 2$ in the inverted orientation resulting in a physiologically favorable condition. $^{13}$

To induce this physiologic motion response in the cervical spine, a custom designed single actuator-adaptable programmable testing system was used. The testing apparatus, shown in Figure 2-1, contained an upper fixture, consisting of a linear bearing and splined shaft assembly, which attached through a rotational joint to a vertical actuator (International Device Corp., Novato, CA). ${ }^{8}$ The actuator applied a compressive load and bending moment to the specimen at a $200 \mathrm{~mm}$ offset from the specimen's long axis. As mentioned previously, the specimen was mounted in a neutral inverted orientation with the T1 pot attached to the upper fixture and the $\mathrm{C} 2$ pot attached to the base. Global rotation was measured by a rotational displacement transducer (Data Instruments, Acton, MA), attached to the upper fixture. In lateral bending, specimens were permitted to rotate axially due to the physiological coupling between lateral bending and axial rotation. ${ }^{47}$ For axial rotation, shown in Figure 2-2, the base was free to tilt laterally as well as rotate axially. The upper mounting fixture was unconstrained in the vertical direction. All specimens were loaded under displacement control for all testing modes.

Segmental cervical motion was tracked using a real time non-contact three-dimensional measurement system. Six target arrays containing light emitting diodes were rigidly fixed to the individual spinous processes to allow for two-dimensional motion analysis. These targets 


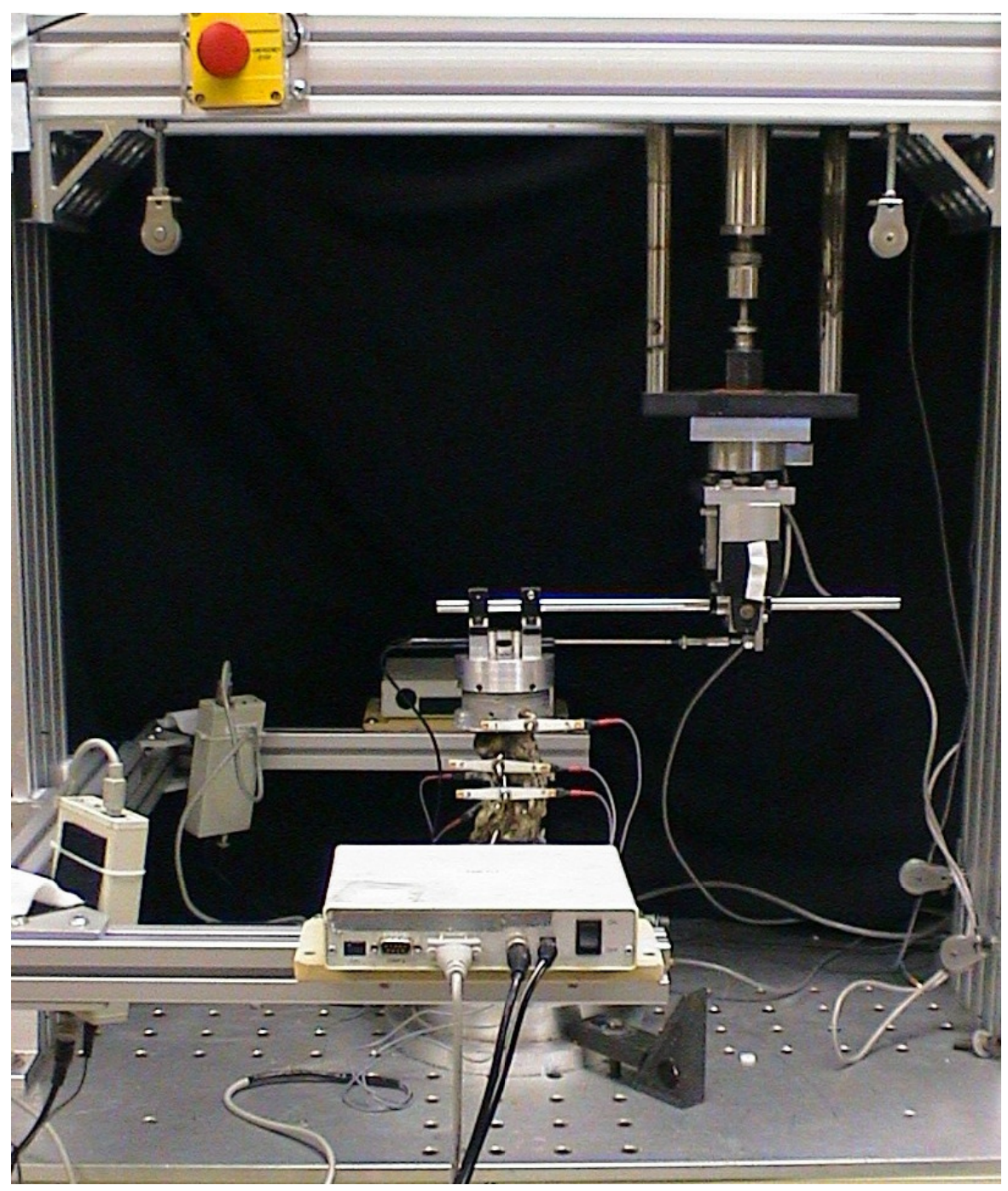

Figure 2-1: Custom Designed Non-Destructive Biomechanical Testing Machine. Bending testing set-up shown here, with spine prepared for flexion. 


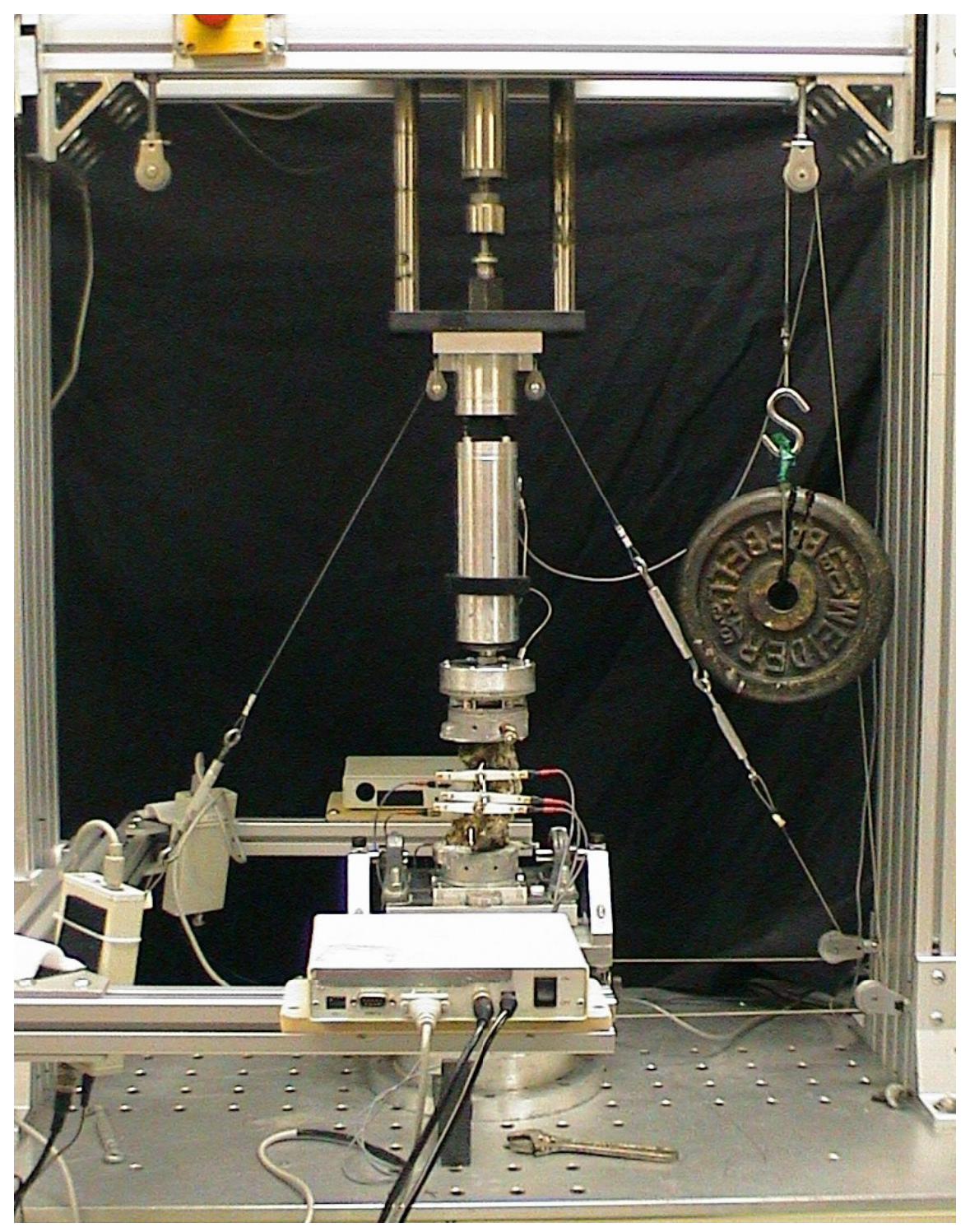

Figure 2-2: Axial Testing. 
uniquely described the position of the vertebral body in a global reference frame defined by the camera receiver unit. The motion segment units (MSU) were described relative to the adjacent vertebral body using rigid body mechanics.

\subsection{Data Management and Statistical Analysis}

As described above, relative motion of each MSU was recorded using custom LabView (National Instruments) programming software. All data were normalized to the respective harvested specimen by dividing either the fused results or arthroplasty results by the respective harvested results to determine normalized flexibility, normalized motion, and change in MSU percent contribution values. Mean normalized values were calculated, grouped by instrumented condition, and displayed in bar graphs relative to the non-instrumented condition, which was graphically represented by unity. Global moment values were uniform across the three instrumented conditions for each loading mode studied.

Flexibility values were defined as the global rotation of the specimen divided by the global moment of the loading condition (flexion, extension, etc.) at a common end limit of global moment for all cases. Flexibility values were normalized by comparing the instrumented flexibility (fusion or disc arthroplasty) to non-instrumented flexibility (harvested). Normalizing the values by comparing the two-level disc arthroplasty or two-level fusion condition to the harvested condition reduces error due to tissue variability by establishing each value relative to the spine and loading condition studied.

Normalized motion, shown in Equation 2-1, compares operative levels (C5-C6-C7) relative motion of the fusion or disc arthroplasty condition to the relative motion at the harvested 
condition at those same levels. The relative motion of the operated levels is the motion of levels C5-C6-C7 divided by the global motion of the entire spine for each condition.

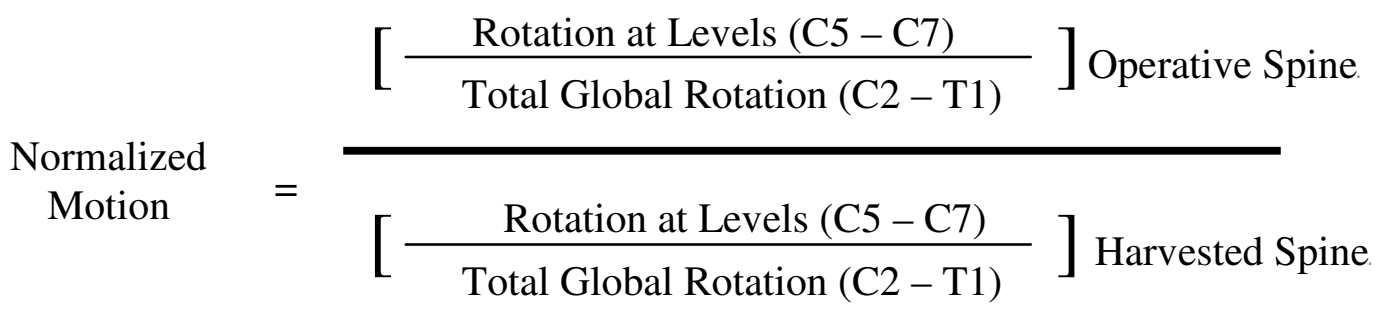

Eq. $2-1$

MSU percent change in contribution compares the percent motion contribution of each operative condition at a particular MSU to the percent motion contribution of the harvested condition at that same MSU. The percent motion contribution is the motion contribution at a particular MSU divided by the total global motion of the specimen expressed as a percentage as shown in Equation 2-2 with a visual representation in Figure 2-3.

$$
\left[\frac{\text { MSU Relative Motion at One Level }}{\text { Specimen Total Global Motion }}\right] \text { Operative Spine }
$$

Percent Change in $=$ one MSU

$$
\left[\frac{\text { MSU Relative Motion at One Level }}{\text { Specimen Total Global Motion }}\right] \text { Harvested Spine }
$$

Statistical analysis utilized one-way analysis of variance (ANOVA) to determine if a difference existed among the three groups (harvested, disc arthroplasty, and fusion). Values detected as significantly different were further analyzed for pair wise comparison by Student Newman Keuls (SNK) test. Significance was defined as $\mathrm{P}<0.05$ unless otherwise stated. Normality and equal variance was assumed for all groups. 

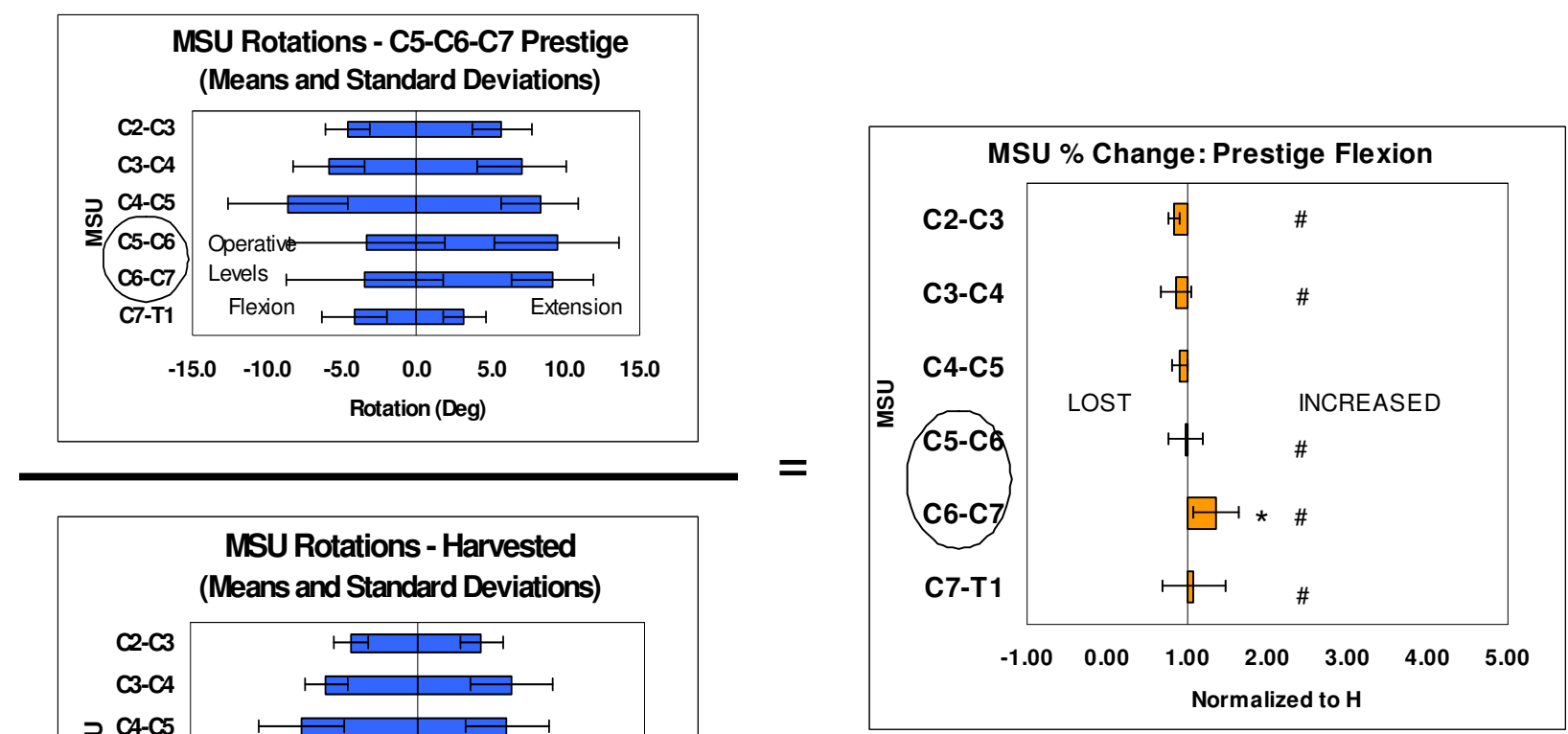

Figure 2-3: MSU Percent Change in Contribution Calculation. All percent change in contribution values were normalized to harvested $(\mathrm{H})$, represented by unity. An increase from unity indicates an increase in the segment motion contribution relative to the motion in the harvested condition, and a decrease from unity indicates a decrease in motion contribution for the instrumented condition relative to the harvested condition. In this case total rotation of the harvested condition and the Prestige-LP condition were equivalent. * Signifies significant difference from harvested $(\mathrm{H})$. \# Signifies significant difference between fusion and disc arthroplasty. 


\section{Prestige-LP Cervical Arthroplasty Versus Fusion at Two Levels: What Are the Biomechanical Differences?}

\subsection{Introduction}

Anterior cervical discectomy and fusion (ACDF) surgery is the standard of care for treatment of end stages of degenerative disc diseases of the cervical spine. However it has been widely documented that spinal decompression and fusion procedures in the cervical spine are likely to cause adjacent segment disease, a reoccurrence of degenerative disc disease above or below the operated level. ${ }^{3,9,16,18,22,24,27,31,36,38,41,48}$ Similar results have been reported for cases of multi-level fusion of the cervical spine. ${ }^{21,24,27}$ An alternative treatment for patients intended to alleviate complications of adjacent segment disease is prosthetic disc replacement surgery. In an early clinical study that compared fusion and prosthetic disc patient populations, a significant difference in motion at adjacent segments between the two groups was reported. ${ }^{49}$ While increasing reports of two-level artificial disc implantation have begun to surface in the clinical literature, ${ }^{4,15,19,33,44}$ no biomechanical evaluation of this procedure exists. The objective of this study was to determine in vitro biomechanics of a two-level (C5-C6 and C6-C7) disc arthroplasty using an established cadaveric model. The two-level arthroplasty model was compared to a two-level fusion condition and the harvested condition. 


\subsection{Materials and Methods}

\subsubsection{Specimen Preparation and Spinal Conditions}

Six fresh human cadaveric sub-axial (C2-T1) cervical spines with a mean age of $63.2 \pm$ 5.9 years, were harvested, wrapped in saline soaked gauze, placed in double plastic bags, and frozen at negative $20^{\circ} \mathrm{C}$. Prior to preparation, specimens were thawed in a standard refrigeration system for 12 hours. Anterior-posterior and lateral radiographs were used to exclude any specimen with gross osteopenia or anatomic abnormality.

Specimen preparation included removal of excess musculature, with preservation of spinal ligaments, discs, and bone, as well as additional tissue removal at $\mathrm{C} 2$ and $\mathrm{T} 1$ to expose boney surfaces for potting material purchase. Screws were used as additional fixation at the spinal processes, exposed facet joints, and exposed vertebral bodies (VB). Positioning screws passing through the sides of the mounting pots initially held the end vertebral bodies in place, while a low-melting-point bismuth alloy (Small Parts, Miami Lakes, FL) was poured into the pots to provide final fixation. Threaded rods were inserted into the lateral aspects of the VB's for motion tracking target attachment and external fusion fixation. These rods did not interfere with device installation.

Testing conditions included: the harvested or non-instrumented condition (harvested) two-level fusion (fusion) at levels C5-C6 and C6-C7, and two-level disc arthroplasty at spinal levels C5-C6 and C6-C7. The Prestige Low-Profile (LP) cervical disc replacement device (Medtronic Sofamor Danek, Memphis, TN) was used for the disc arthroplasty condition (Prestige-LP), as shown in Figure 3-1. Fusion was simulated using customized fixtures similar to an external fixation system used by orthopedic surgeons. The different spine conditions are shown in Figure 3-2. 


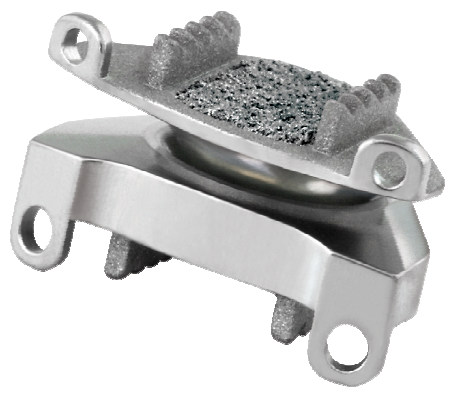

Figure 3-1: Prestige Low-Profile Disc Prosthesis. Initial fixation is provided by two keels that engage in the vertebral body end plates. The articulating components are made of a titanium ceramic composite material, and the theoretical center of rotation is located on the superior VB. (Used with permission by Medtronic Sofamor Danek.)

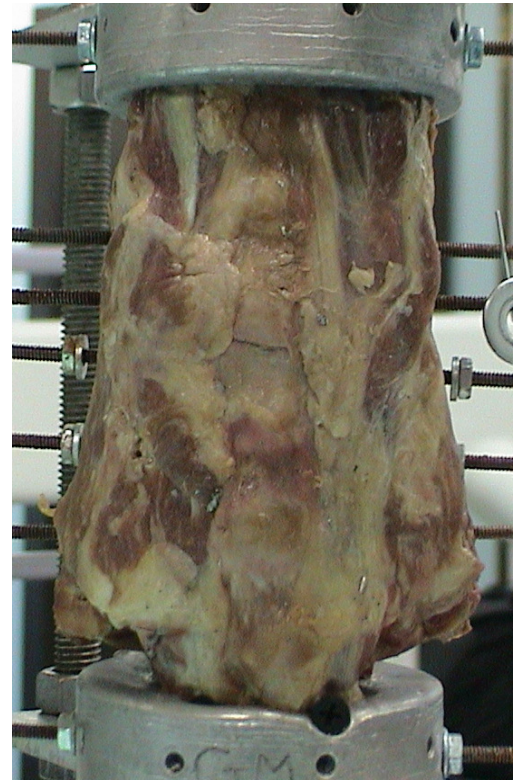

a

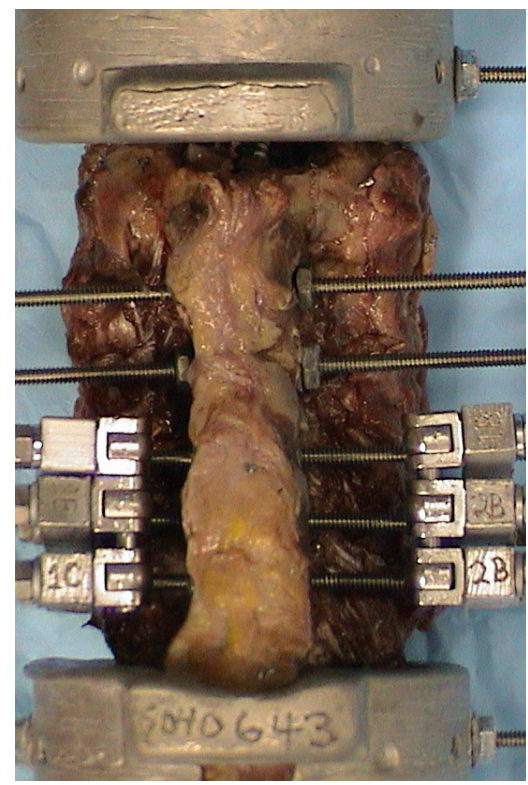

b

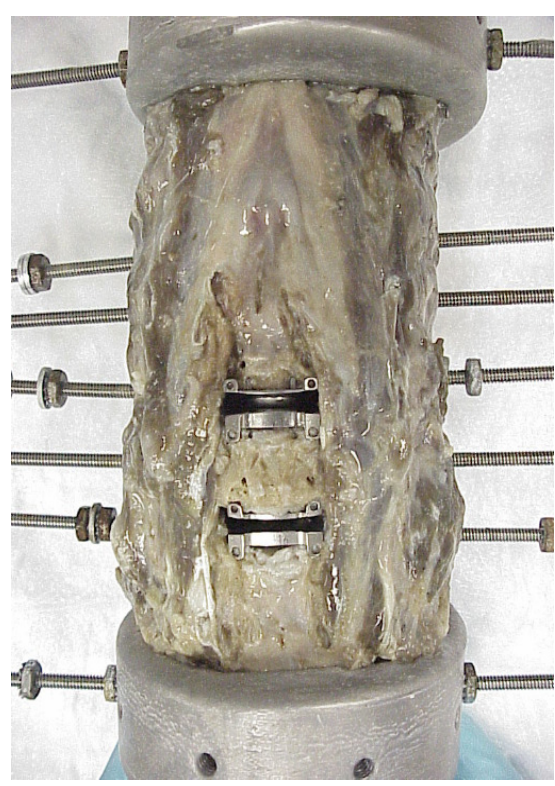

c

Figure 3-2: Test Prepared Specimens. (a) The non-instrumented control or harvested specimen. (b) Specimen instrumented with AO clamps and rods simulating external two-level fusion. (c) Two-level disc arthroplasty using Prestige-LP disc replacement device. 


\subsubsection{Non-Destructive Testing Protocol}

An existing testing protocol designed to simulate in vivo cervical spine kinematics was used to test the spines in flexion-extension, left/right lateral bending, and left/right axial rotation ${ }^{8,}$ ${ }^{12}$ This protocol has been used previously to evaluate cervical fusion techniques ${ }^{14,40}$ as well as single level prosthetic disc devices. ${ }^{13,14}$ Due to the strong coupling of axial rotation with lateral bending, the spines were unconstrained in axial rotational during lateral bending tests, and unconstrained in lateral bending during axial rotation tests. Flexion/extension and lateral bending test set-up is shown in Figure 3-3.

All tests were performed under displacement control with the spine positioned at a 200 $\mathrm{mm}$ offset distance from the actuator load axis. The testing apparatus was programmed to output a triangular shaped displacement-time waveform at an actuator velocity of $6.4 \mathrm{~mm} / \mathrm{sec}$, corresponding to approximately $2.0 \mathrm{deg} / \mathrm{sec}$ global spinal rotation. Specimens were tested to a global moment of $3.5 \mathrm{Nm}$ unless a global rotation of $45^{\circ}$ was reached. All specimens were pre-

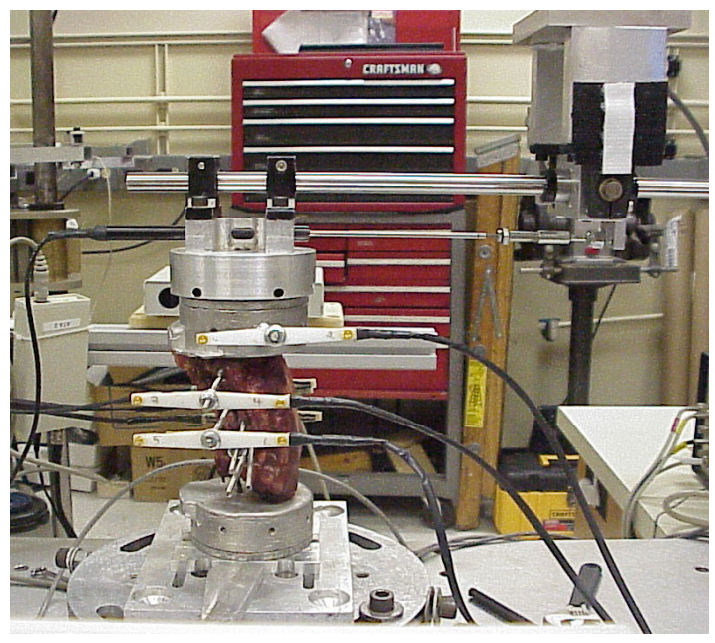

a

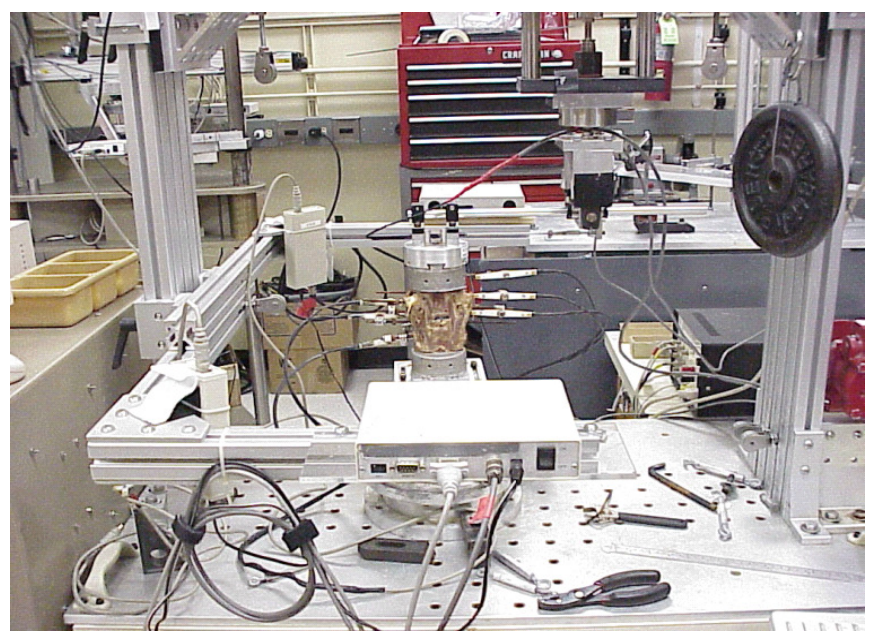

b

Figure 3-3: Biomechanical Testing Apparatus. Testing set-up for (a) flexion and extension and (b) lateral bending. 
conditioned with at least five cycles prior to the final test trial of three consecutive cycles. Specimens were regularly moistened with a normal saline mist throughout preparation and testing.

\subsubsection{Data Management and Statistical Analysis}

Measurements of global spinal movements, applied load, and individual vertebral body motions were collected at $10 \mathrm{~Hz}$. A non-contact measurement system was used to record the three-dimensional segmental cervical motion for each testing condition. Global rotation and applied load data were used to calculate overall spinal flexibility. All motion measurements were analyzed at an end limit of global moment common to all spine conditions within each specimen. All of the instrumented spine conditions were normalized with respect to their harvested condition before comparison to account for intrinsic differences in specimen variability. Motion response at the operated regions were compared by determining the relative contribution of levels $\mathrm{C} 5-\mathrm{C} 7$ to the overall global motion, and then normalizing with respect to the intact spine condition. The same normalization procedure was used to compare percent changes in individual MSU rotations at the remaining non-operated spinal levels for the instrumented spine conditions. MSU percent change in contribution compares the percent motion contribution of the operative condition at a particular MSU to the percent motion contribution of the harvested condition at that same MSU. The percent motion contribution is the motion contribution at a particular MSU divided by the total global motion of the specimen expressed as a percentage. Statistical analysis utilized one-way analysis of variance (ANOVA). If a significant different between groups was detected, the Student Newman Keuls (SNK) test was used for pair wise comparison. Significance was defined as $\mathrm{P}<0.05$. For all groups, normality and equal variance was assumed. 


\subsection{Results}

\subsubsection{Normalized Flexibility}

The global motion versus rotation data of the instrumented and harvested spine conditions are shown in Table 3-1. The flexibility values for the fused and implanted conditions were normalized to the harvested condition and compared at a common end limit of moment as shown in Figure 3-4. Fusion significantly decreased the normalized flexibility in all testing modes compared to the harvested condition, except in right axial rotation. The fused spine condition was also significantly different from the two-level Prestige-LP spine condition in all tested modes except left axial rotation. The two-level Prestige-LP condition increased the flexibility of the spine relative the harvested condition in extension $(158 \pm 43 \%$ of $\mathrm{H})$, left axial rotation $(114 \pm 14 \%$ of $\mathrm{H})$, and right axial rotation $(123 \pm 24 \%$ of $\mathrm{H})$.

\subsubsection{Normalized Motion}

The MSU rotational data of the treated (C5-C7) levels are shown in Table 3-2 for the three different spine conditions. The rotations at the treated levels were expressed relative to the total spine rotation for the fused and disc arthroplasty conditions and normalized to their respective harvested condition, as shown in Figures 3-5, 3-6, 3-7. A normalized value identical to the harvested condition equals one and can be expressed as being equivalent to $100 \%$ of the harvested condition. There were no significant differences between the Prestige-LP and harvested conditions, except in flexion (114 $\pm 14 \%$ of $\mathrm{H})$, extension $(126 \pm 21 \%$ of $\mathrm{H})$, and flexion plus extension $(117 \pm 7 \%$ of $\mathrm{H})$. A significant decrease in normalized motion occurred between the fused condition and both the harvested and disc arthroplasty spine conditions for all loading modes. 
Table 3-1: Global Rotation Versus Applied Moment Data.

\begin{tabular}{|c|c|c|c|c|c|}
\hline Test Mode & $\begin{array}{c}\text { Spine } \\
\text { Condition }\end{array}$ & $\begin{array}{c}\text { Global } \\
\text { Moment } \\
(\mathrm{Nm})\end{array}$ & $\begin{array}{c}\text { Global } \\
\text { Rotation } \\
\text { (Deg) }\end{array}$ & $\begin{array}{c}\text { Relative } \\
\text { Flexibility } \\
\text { (Deg/Nm) }\end{array}$ & $\begin{array}{l}\text { Normalized } \\
\text { Flexibility }\end{array}$ \\
\hline \multirow{3}{*}{ Flexion } & Harvested & 3.0 & $37.2 \pm 7.1$ & $12.5 \pm 2.8$ & 1.0 \\
\hline & Prestige-LP & 3.0 & $35.9 \pm 9.1$ & $12.2 \pm 3.5$ & $0.97 \pm 0.11$ \\
\hline & Fusion & 3.0 & $27.0 \pm 6.8$ & $9.2 \pm 2.7$ & $0.73 \pm 0.08 * \#$ \\
\hline \multirow{3}{*}{ Extension } & Harvested & 1.7 & $28.7 \pm 7.4$ & $23.1 \pm 13.5$ & 1.0 \\
\hline & Prestige-LP & 1.7 & $43.1 \pm 8.3$ & $35.6 \pm 19.5$ & $1.55 \pm 0.41 * \#$ \\
\hline & Fusion & 1.7 & $20.2 \pm 4.9$ & $15.7 \pm 8.6$ & $0.70 \pm 0.08 * \#$ \\
\hline \multirow{3}{*}{ Left Lateral } & Harvested & 3.1 & $29.4 \pm 6.4$ & $9.5 \pm 2.0$ & 1.0 \\
\hline & Prestige-LP & 3.1 & $30.1 \pm 6.2$ & $9.9 \pm 2.1$ & $1.05 \pm 0.04$ \\
\hline & Fusion & 3.1 & $25.9 \pm 5.7$ & $8.4 \pm 1.8$ & $0.90 \pm 0.10 * \#$ \\
\hline \multirow{3}{*}{ Right Lateral } & Harvested & 3.1 & $28.7 \pm 6.3$ & $9.4 \pm 2.1$ & 1.0 \\
\hline & Prestige-LP & 3.1 & $28.6 \pm 6.4$ & $9.3 \pm 2.0$ & $1.00 \pm 0.08$ \\
\hline & Fusion & 3.1 & $23.8 \pm 5.1$ & $7.8 \pm 1.7$ & $0.84 \pm 0.09 * \#$ \\
\hline \multirow{3}{*}{ Left Axial } & Harvested & 3.1 & $20.7 \pm 4.1$ & $5.9 \pm 1.2$ & 1.0 \\
\hline & Prestige-LP & 3.1 & $24.7 \pm 3.9$ & $6.8 \pm 2.1$ & $1.14 \pm 0.14 * \#$ \\
\hline & Fusion & 3.1 & $18.0 \pm 2.7$ & $5.2 \pm 1.2$ & $0.89 \pm 0.13$ \\
\hline \multirow{3}{*}{ Right Axial } & Harvested & 3.1 & $38.4 \pm 8.1$ & $6.8 \pm 1.4$ & 1.0 \\
\hline & Prestige-LP & 3.1 & $45.3 \pm 9.1$ & $8.1 \pm 1.2$ & $1.21 \pm 0.24 * \#$ \\
\hline & Fusion & 3.1 & $34.2 \pm 6.4$ & $5.9 \pm 0.9$ & $0.88 \pm 0.08$ \\
\hline
\end{tabular}

* Signifies a significant difference relative to the harvested condition.

\# Signifies a significant difference between the fusion condition and the Prestige-LP condition. 


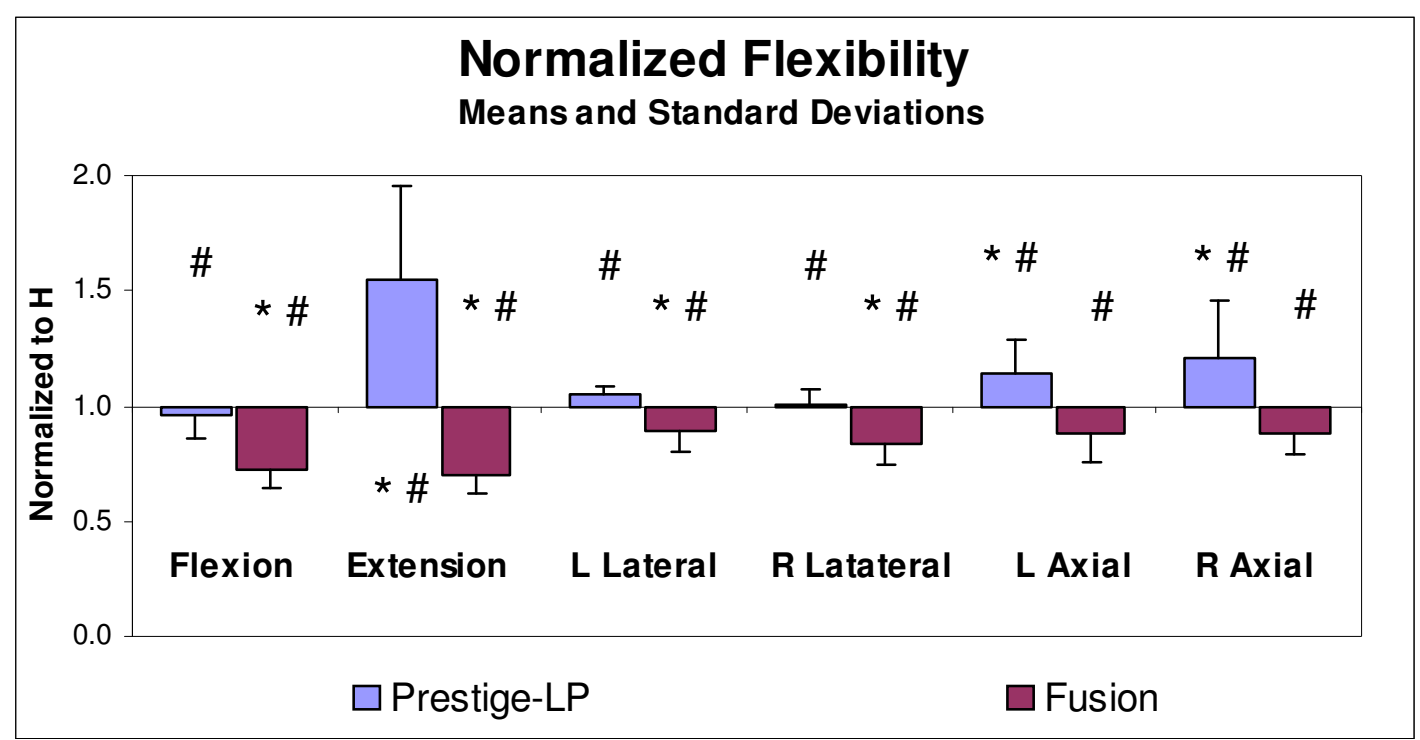

Figure 3-4: Prestige-LP Normalized Flexibility for All Loading Cases. * Signifies a significant difference relative to the harvested condition. \# Signifies a significant difference between the fusion condition and the Prestige-LP condition. 
Table 3-2: Motion at Treated Level.

\begin{tabular}{cccccc}
\hline Test Mode & $\begin{array}{c}\text { Spine } \\
\text { Condition }\end{array}$ & $\begin{array}{c}\text { Global } \\
\text { Motion } \\
\text { (Deg) }\end{array}$ & $\begin{array}{c}\text { Motion at } \\
\text { Treated } \\
\text { Levels (Deg) }\end{array}$ & $\begin{array}{c}\text { Relative } \\
\text { Motion }\end{array}$ & $\begin{array}{c}\text { Normalized } \\
\text { Motion }\end{array}$ \\
\hline \multirow{3}{*}{ Flexion } & Harvested & $37.2 \pm 7.1$ & $15.7 \pm 4.7$ & $0.42 \pm 0.08$ & 1.0 \\
& Prestige-LP & $35.9 \pm 9.1$ & $16.9 \pm 5.0$ & $0.47 \pm 0.06$ & $0.40 \pm 0.56 * \#$ \\
& Fusion & $27.0 \pm 6.8$ & $0.4 \pm 0.2$ & $0.02 \pm 0.01$ & $0.04 \pm 0.23 * \#$ \\
\hline \multirow{2}{*}{ Extension } & Harvested & $28.7 \pm 7.4$ & $9.4 \pm 0.6$ & $0.35 \pm 0.08$ & 1.0 \\
& Prestige-LP & $43.1 \pm 8.3$ & $18.7 \pm 6.0$ & $0.43 \pm 0.11$ & $1.26 \pm 0.21 * \#$ \\
& Fusion & $20.2 \pm 4.9$ & $0.6 \pm 0.4$ & $0.03 \pm 0.02$ & $0.09 \pm 0.05 * \#$ \\
\hline \multirow{2}{*}{ Flexion + } & Harvested & $65.8 \pm 13.5$ & $25.2 \pm 4.9$ & $0.39 \pm 0.05$ & 1.0 \\
& Prestige-LP & $79.0 \pm 13.9$ & $35.6 \pm 7.5$ & $0.45 \pm 0.06$ & $1.17 \pm 0.07 * \#$ \\
& Fusion & $47.2 \pm 11.4$ & $1.0 \pm 0.3$ & $0.02 \pm 0.01$ & $0.06 \pm 0.02 * \#$ \\
\hline \multirow{2}{*}{ Left Lateral } & Harvested & $29.4 \pm 6.4$ & $9.2 \pm 4.1$ & $0.30 \pm 0.10$ & 1.0 \\
& Prestige-LP & $30.1 \pm 6.2$ & $10.5 \pm 4.8$ & $0.34 \pm 0.11$ & $1.12 \pm 0.11 \quad \#$ \\
& Fusion & $25.9 \pm 5.7$ & $0.6 \pm 0.4$ & $0.02 \pm 0.02$ & $0.09 \pm 0.08 * \#$ \\
\hline \multirow{2}{*}{ Right Lateral } & Harvested & $28.7 \pm 6.3$ & $9.8 \pm 4.2$ & $0.33 \pm 0.12$ & 1.0 \\
& Prestige-LP & $28.6 \pm 6.4$ & $9.3 \pm 4.2$ & $0.31 \pm 0.12$ & $0.97 \pm 0.20 \quad \#$ \\
& Fusion & $23.8 \pm 5.1$ & $0.7 \pm 0.8$ & $0.03 \pm 0.03$ & $0.09 \pm 0.11 * \#$ \\
\hline \multirow{2}{*}{ Left + Right } & Harvested & $58.1 \pm 12.7$ & $18.9 \pm 8.2$ & $0.32 \pm 0.10$ & 1.0 \\
& Prestige-LP & $58.7 \pm 12.2$ & $19.8 \pm 8.8$ & $0.33 \pm 0.10$ & $1.03 \pm 0.09 \#$ \\
& Fusion & $49.8 \pm 10.7$ & $1.3 \pm 0.7$ & $0.03 \pm 0.10$ & $0.09 \pm 0.05 * \#$ \\
\hline \multirow{2}{*}{ Left Axial } & Harvested & $18.3 \pm 3.8$ & $5.0 \pm 1.4$ & $0.28 \pm 0.08$ & 1.0 \\
& Prestige-LP & $20.7 \pm 6.2$ & $6.1 \pm 2.5$ & $0.29 \pm 0.07$ & $1.07 \pm 0.20 \#$ \\
& Fusion & $16.2 \pm 3.8$ & $0.8 \pm 0.6$ & $0.05 \pm 0.04$ & $0.21 \pm 0.15 * \#$ \\
\hline \multirow{2}{*}{ Right Axial } & Harvested & $20.7 \pm 4.1$ & $5.4 \pm 1.9$ & $0.27 \pm 0.08$ & 1.0 \\
& Prestige-LP & $24.7 \pm 3.9$ & $7.1 \pm 2.9$ & $0.28 \pm 0.09$ & $1.06 \pm 0.20 \#$ \\
& Fusion & $18.0 \pm 2.7$ & $1.0 \pm 0.5$ & $0.05 \pm 0.03$ & $0.21 \pm 0.15 * \#$ \\
\hline \multirow{2}{*}{ Left + Right } & Harvested & $38.4 \pm 8.1$ & $10.2 \pm 3.0$ & $0.27 \pm 0.07$ & 1.0 \\
Axial & Prestige-LP & $45.3 \pm 9.1$ & $12.9 \pm 5.3$ & $0.28 \pm 0.09$ & $1.05 \pm 0.08 \quad \#$ \\
& Fusion & $34.2 \pm 6.4$ & $1.7 \pm 1.0$ & $0.05 \pm 0.03$ & $0.21 \pm 0.16 * \#$ \\
\hline & & & & &
\end{tabular}

* Signifies significant difference from the harvested $(\mathrm{H})$ condition.

\# Signifies significant difference between the fusion condition and the Prestige-LP condition. 


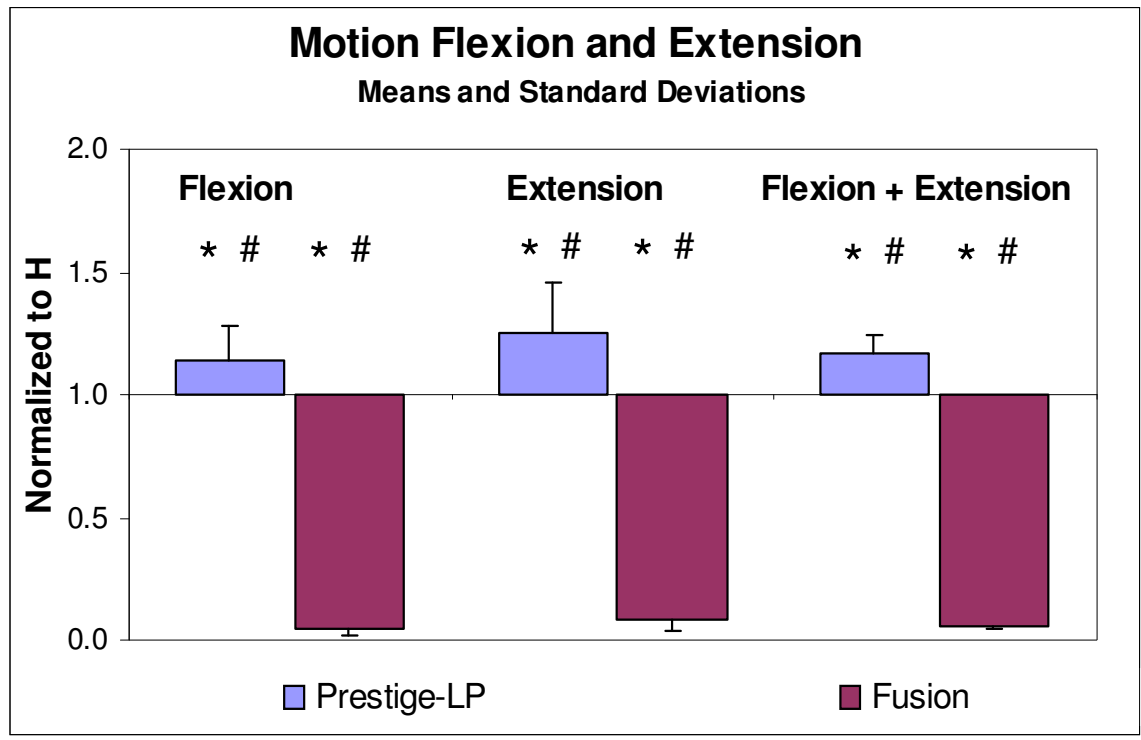

Figure 3-5: Prestige-LP Normalized Motion in Flexion and Extension. * Signifies significant difference from the harvested $(\mathrm{H})$ condition. \# Signifies significant difference between the fusion condition and the Prestige-LP condition.

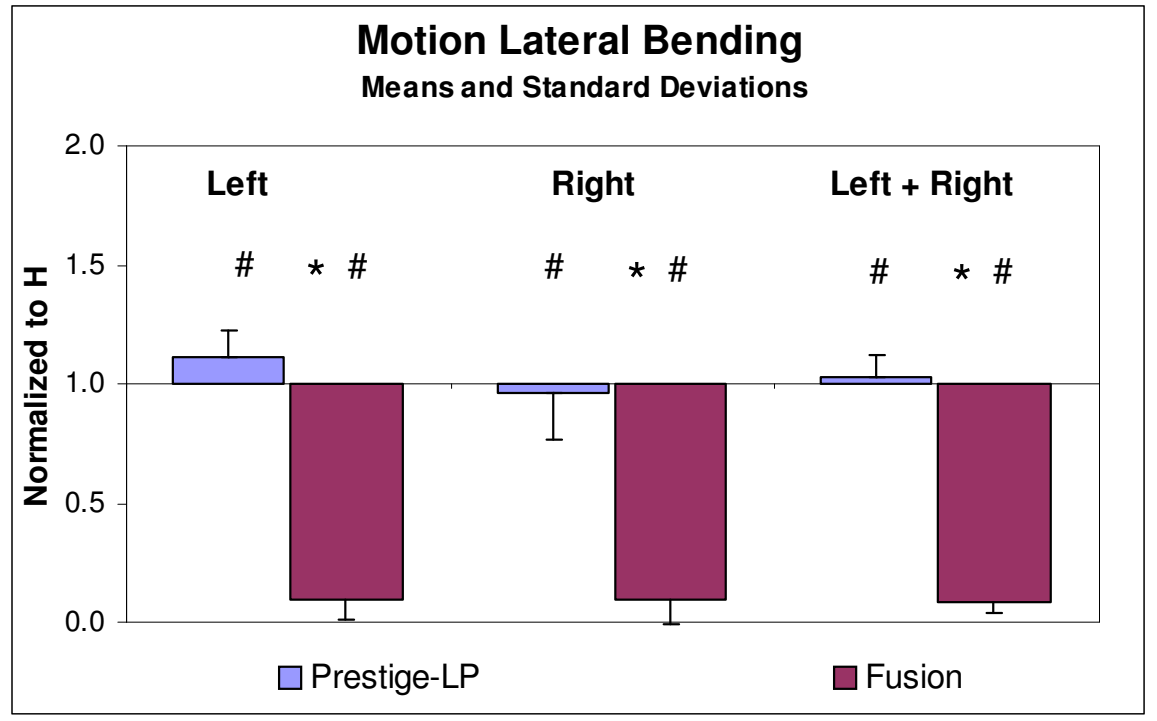

Figure 3-6: Prestige-LP Normalized Motion in Lateral Bending. All values relative to unity, representing harvested $(\mathrm{H})$. * Signifies a significant difference from harvested. \# Signifies significant difference between Prestige-LP and fusion. 


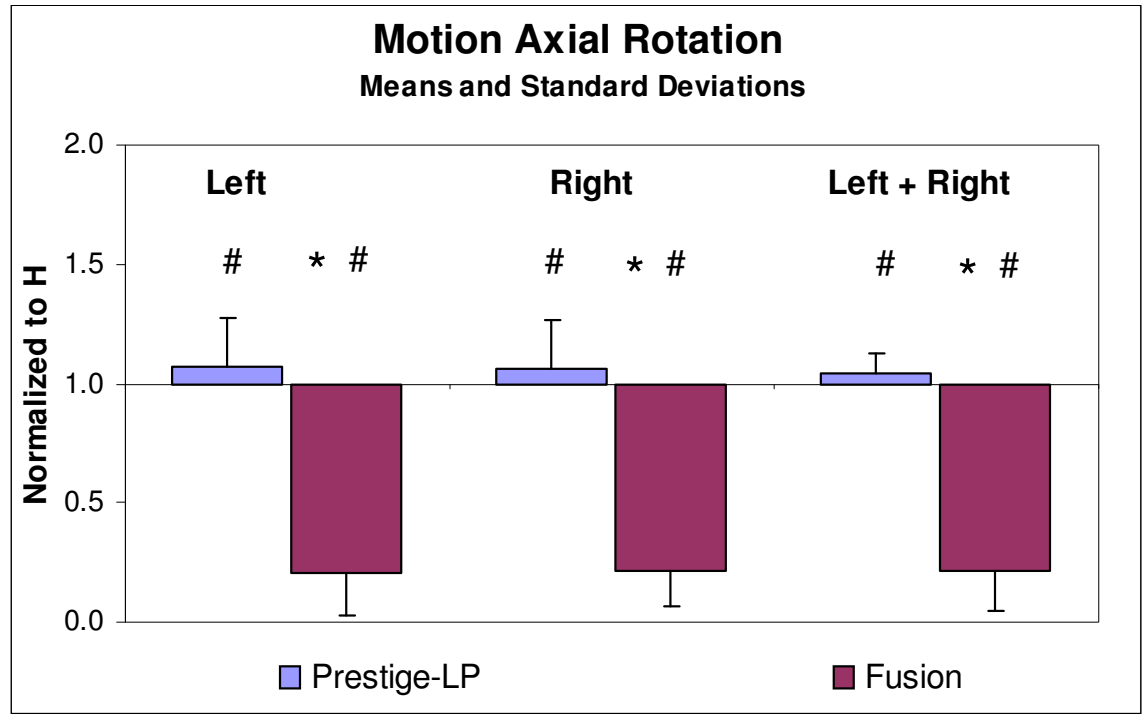

Figure 3-7: Prestige-LP Normalized Motion in Axial Rotation. * Signifies significant difference from the harvested $(\mathrm{H})$ condition. \# Signifies significant difference between PrestigeLP and fusion.

\subsubsection{MSU Rotation Contribution}

Relative MSU rotation values for each spine condition and loading scenarios are shown in Figure 3-8. The percent change in the contribution of motion at the treated levels (C5-C6 and C6-C7) of the fused and implanted conditions relative the harvested contribution is shown in Figures 3-9, 3-10, and 3-11. A significant decrease in motion occurred between the fused condition and both the harvested and disc implanted conditions for all loading modes. A significant increase in motion occurred between the two-level Prestige and the harvested conditions in extension at C5-C6 and in both flexion and extension at C6-C7.

A significant increase in motion occurred for the fusion condition at the levels immediately adjacent (C4-C5 and C7-T1) to the treated levels in all loading cases, except in right axial rotation at $\mathrm{C} 4-\mathrm{C} 5$, and left axial rotation at $\mathrm{C} 7-\mathrm{T} 1$ compared to the harvested condition. 

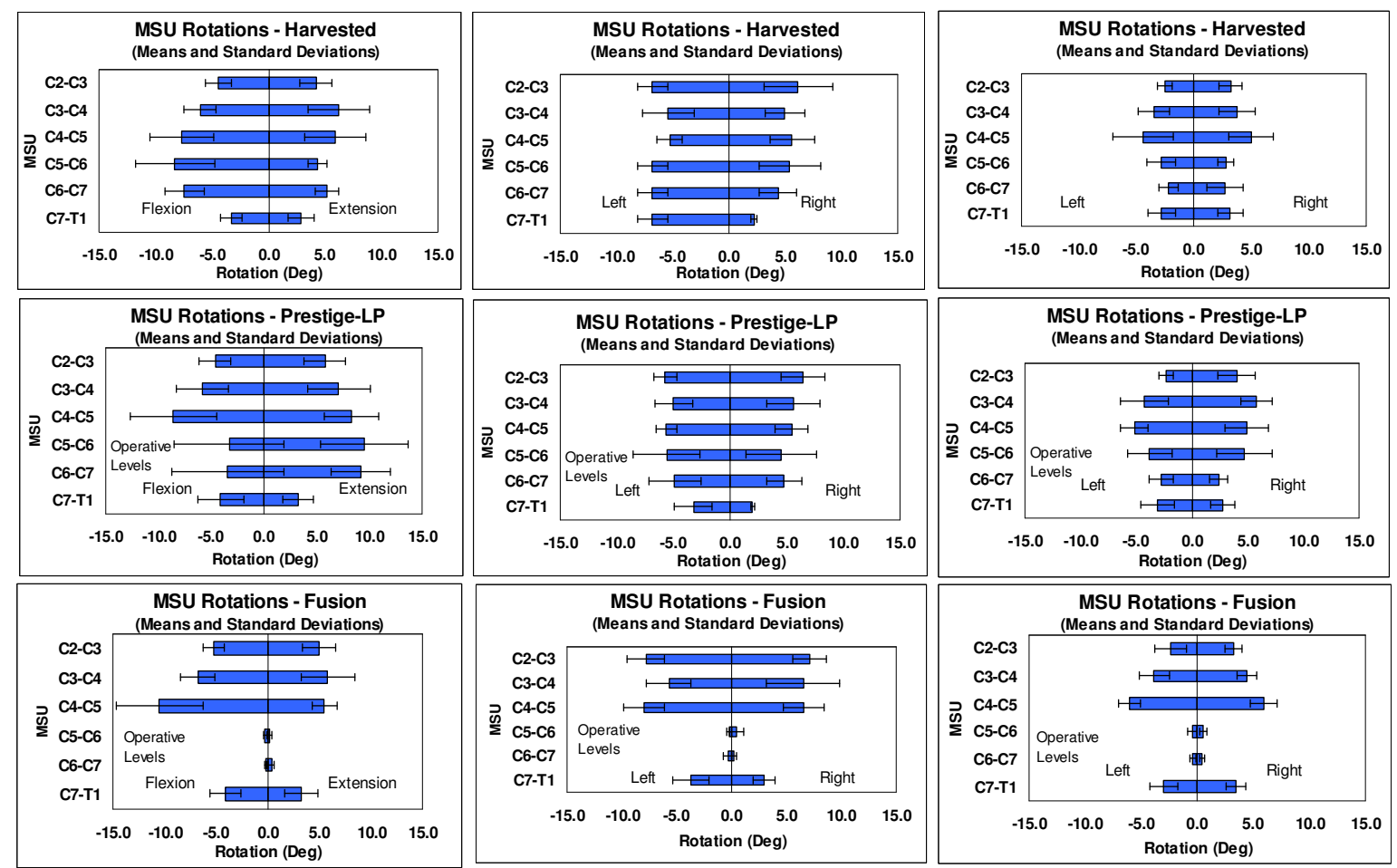

Figure 3-8: Prestige-LP MSU Rotations. Testing modes are in columns: flexion/extension, left/right lateral bending, and left/right axial rotation. The specimen conditions are in rows: harvested, Prestige-LP, and Fusion. Relative MSU rotations were used to calculate the normalized data. 

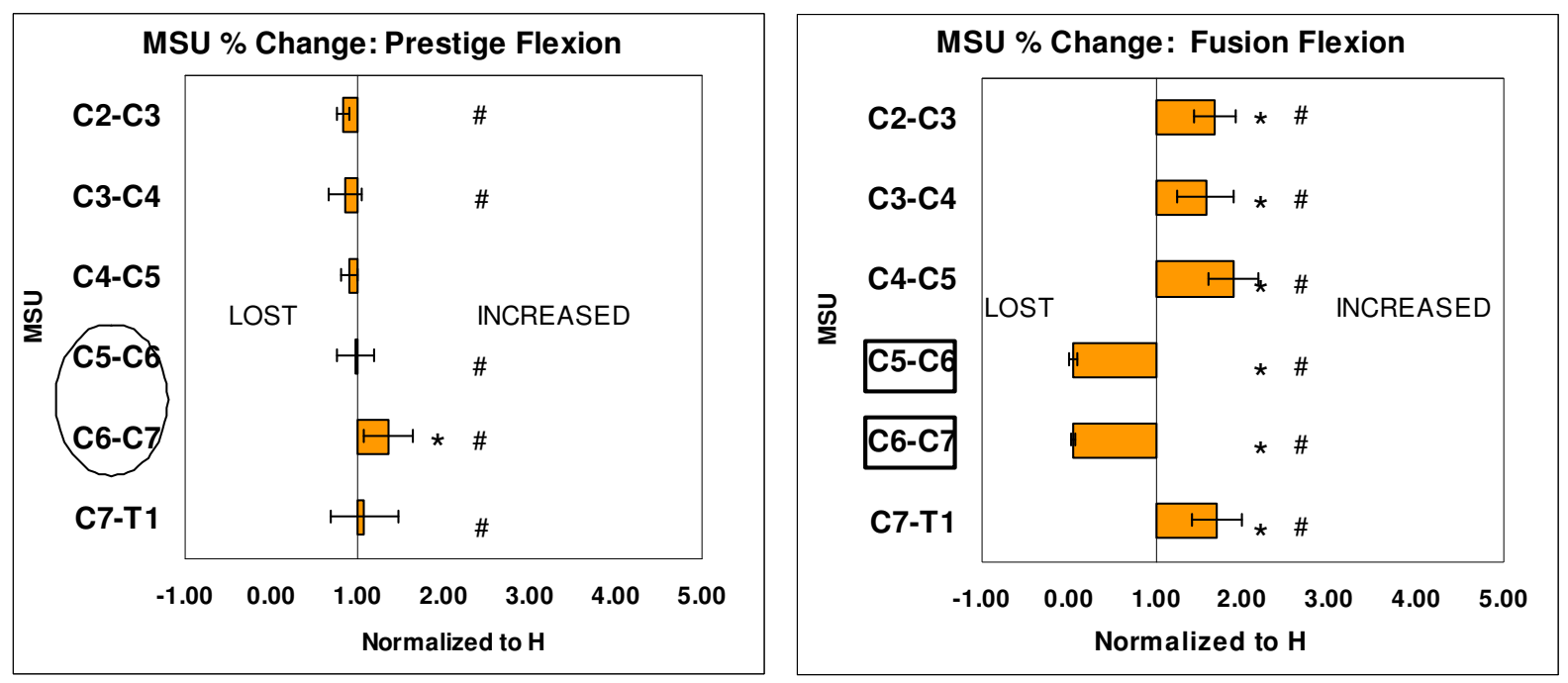

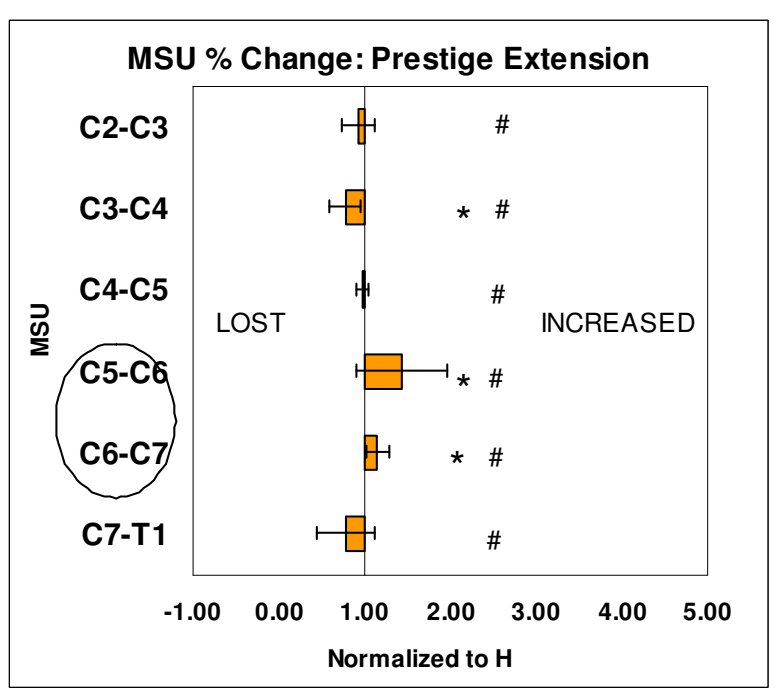

a

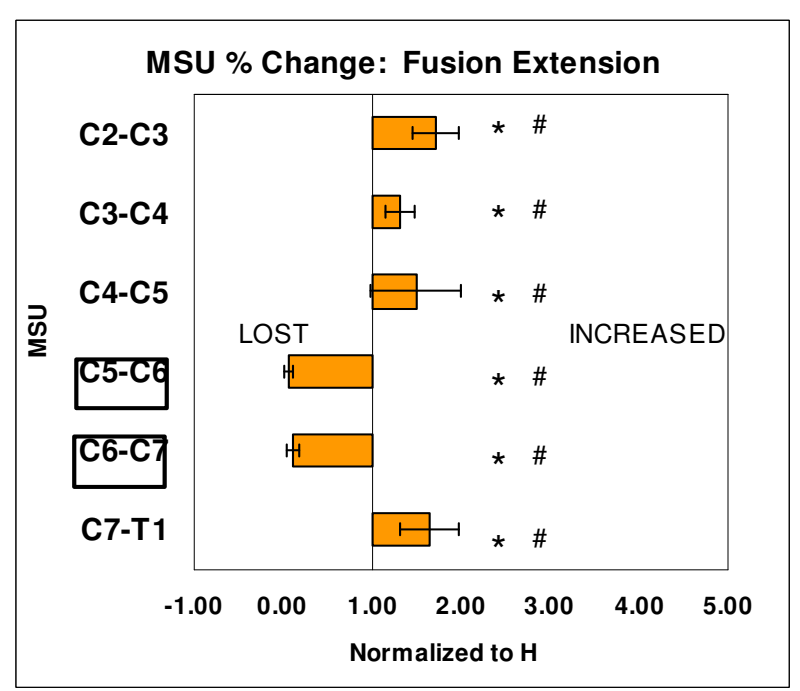

b

Figure 3-9: Prestige-LP MSU Percent Change in Contribution in Flexion and Extension. (a) Prestige-LP and (b) fusion. The * indicates a significant difference relative to the harvested $(\mathrm{H})$ value, and \# indicates a significant difference between the Prestige-LP and fusion conditions. 

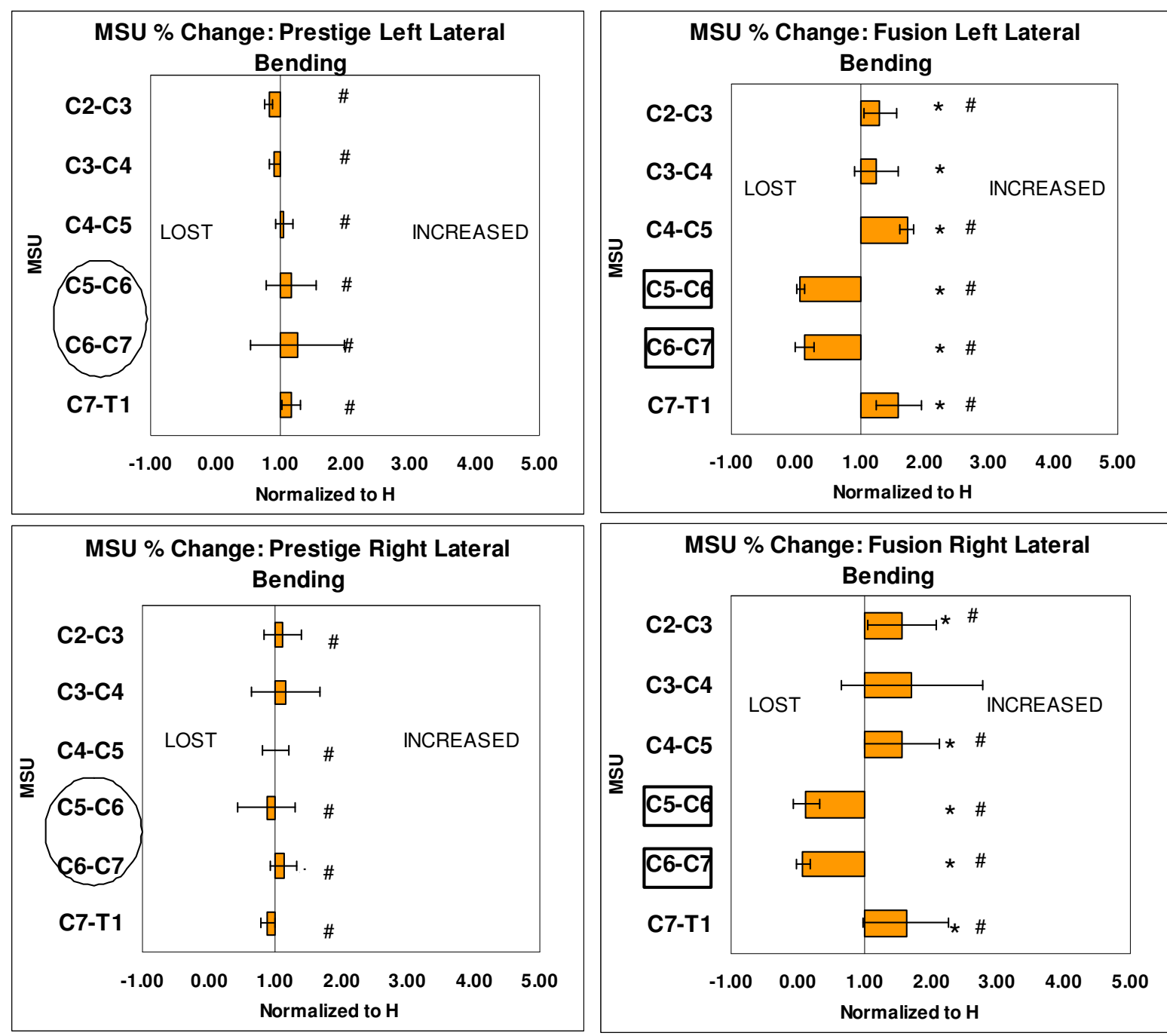

a

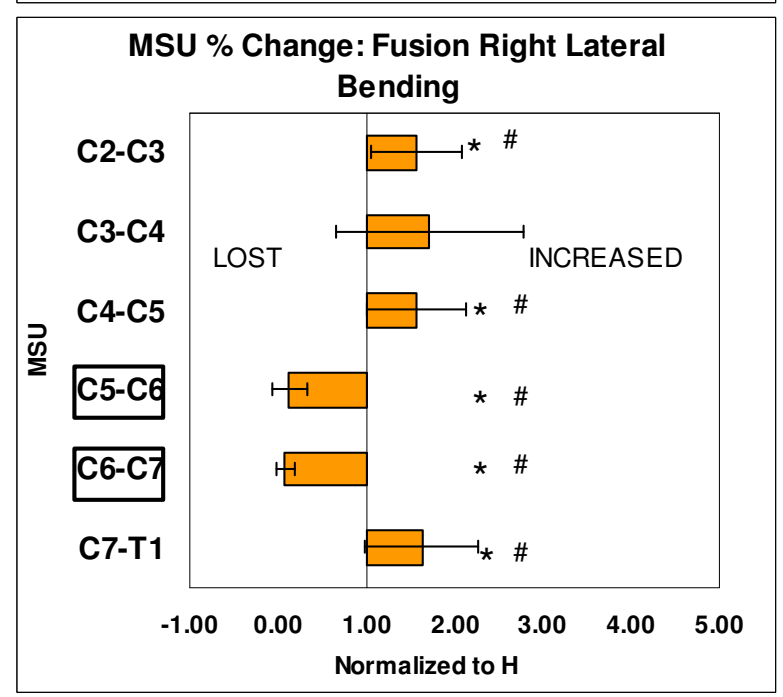

b

Figure 3-10: Prestige-LP MSU Percent Change in Contribution in Lateral Bending. (a) Prestige-LP and (b) fusion conditions. The * indicates a significant difference relative to the harvested $(\mathrm{H})$ value, and \# indicates a significant difference between the Prestige-LP and fusion conditions. 

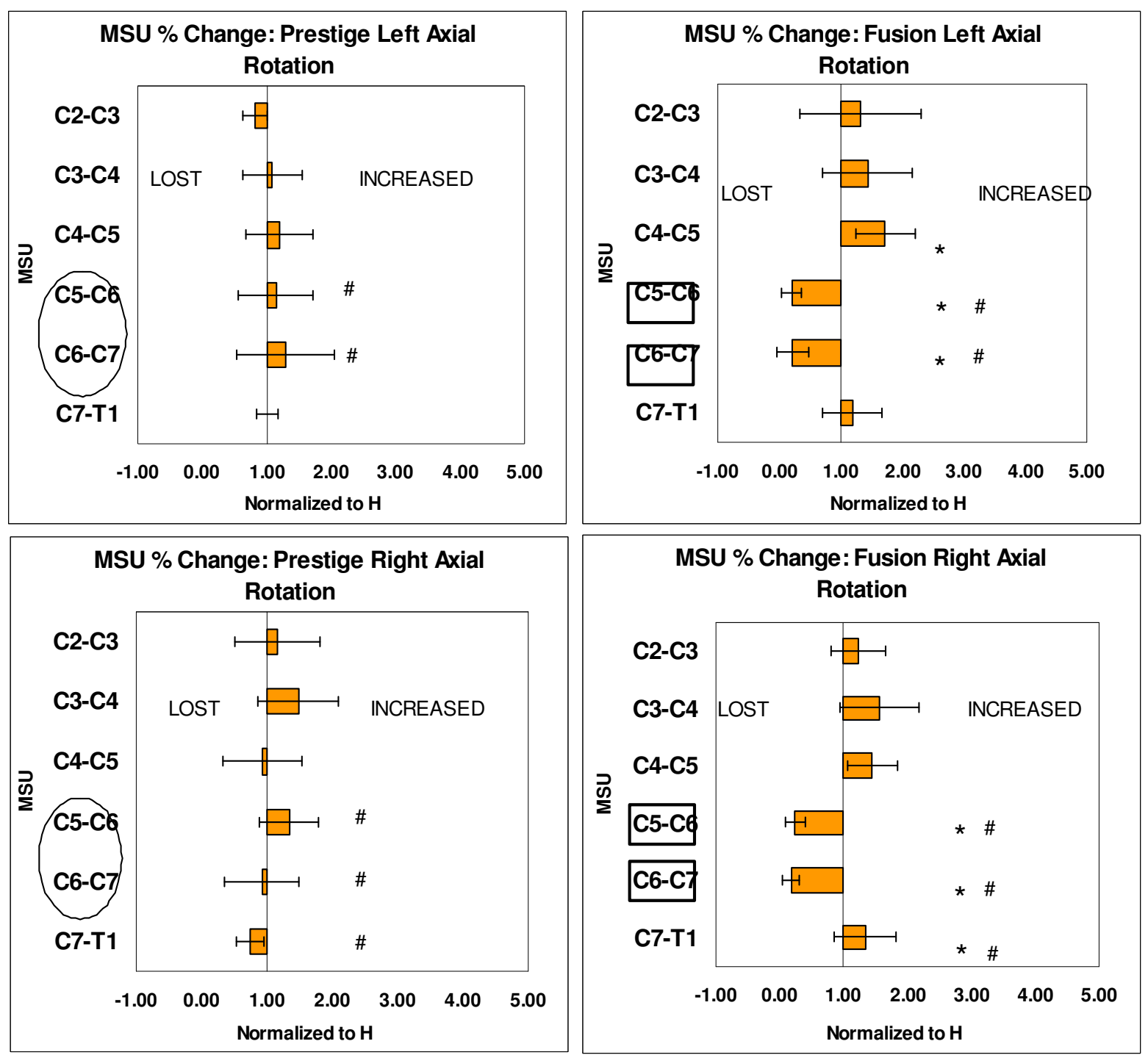

a

b

Figure 3-11: Prestige-LP MSU Percent Change in Contribution in Axial Rotation. (a) Prestige-LP and (b) fusion. The * indicates a significant difference relative to the harvested $(\mathrm{H})$ value, and \# indicates a significant difference between the Prestige-LP and fusion conditions. 
There were no significant differences between Prestige-LP and harvested spine conditions for any loading case at these levels. The fused condition had significantly increased motion as compared to Prestige-LP condition at C4-C5 in all loading conditions except both left and right axial rotation. A significant difference also occurred at C7-T1 between fusion and Prestige-LP in all loading cases except left axial rotation.

\subsection{Discussion}

The purpose of cervical discectomy and arthodesis is to decompress the nerve roots near the spinal column and stabilize the motion segment unit. Stabilization of this nature reduces the motion at the operated levels, but increases the motion at the adjacent levels as seen in biomechanical testing. ${ }^{14,40}$ Significant motion increases at levels adjacent to the operative level

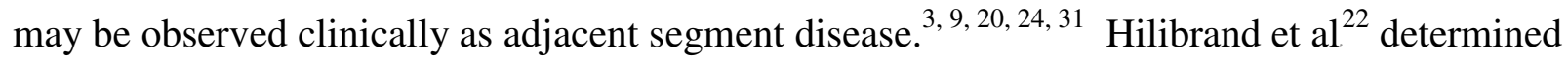
symptomatic adjacent segment disease would potentially affect $25 \%$ of patients within ten years of anterior cervical arthrodesis. However, they found this to be significantly greater in subjects with one-level arthrodesis compared to two-level arthrodesis. In a 21-year average follow up study Gore and Sepic ${ }^{21}$ presented 17 patients with two-level anterior discectomy and fusion. Of these patients $18 \%$ experienced reoccurring pain requiring additional surgery for adjacent segment degeneration. The advantages of an ideal implantable disc prosthesis lie in the ability to replace the native tissue responsible for the symptoms and maintain joint stability, while restoring natural motion to both the affected and adjacent MSUs.

While there is substantial and growing data in the literature related to the single level case, there have been few clinical studies reporting outcomes for two-level cervical arthroplasty. The Bryan Cervical Disc prosthesis clinical trial presented by Goffin et al ${ }^{15}$ followed 26 patients with 
two-level implantation for one year. Effected levels were not specified in this study. At one year patient outcome was $96 \%$ with excellent, good, or fair results. Of those who received twolevel implants, $86 \%$ had a range of motion of $2^{\circ}$ or greater at one year follow-up. Sekhon et al. ${ }^{42}$ used the Bryan Cervical Disc Prosthesis to treat cervical spondylitic myelopathy with an 18 month follow-up. In this study, four of eleven patients were implanted at two levels: two patients with implant levels C4-C5 and C5-C6, and two patients with implant levels C5-C6 and C6-C7. Of these patients three reported an excellent outcome while one reported a fair outcome. An individual with implantation levels $\mathrm{C} 4-\mathrm{C} 5$ and $\mathrm{C} 5-\mathrm{C} 6$ reported the fair outcome.

One range of motion study utilizing static and dynamic digital radiographs by Duggel et al. ${ }^{15}$ included four patients receiving two-level Bryan cervical disc implants. This study collected lateral neutral, flexion, and extension cervical radiographs pre- and post-surgery up to 24 months. While the subjects with two-level implantation were not analyzed as a separate group, range of motion (ROM) was not significantly different post-implantation, suggesting the Bryan prosthetic disc ROM was similar to native disc ROM.

Currently, a multi-center clinical efficacy study is being conducted with the Prestige Low-Profile prosthetic cervical disc. Earlier studies with a previous version, the Prestige II, were conducted with an ACDF control group. ${ }^{35}$ These studies showed increased motion of patients receiving the Prestige II as compared to the ACDF group. However, only patients with one level implantation or fusion were included.

Biomechanical evaluations of two-level disc arthroplasty also remain sparse in the literature. In the current study two-level Prestige Low-Profile disc prosthesis was evaluated and compared to the harvested and instrumented two-level fusion condition in an established cadaveric cervical spine model. The two-level Prestige disc implantation condition was 
statistically more similar to harvested condition than to two-level fusion. Two-level Prestige was not found significantly different from harvested condition except in a few cases such as an increased normalized motion in flexion, extension, and combined flexion and extension. Neverthe-less, the motion increase of the two-level Prestige-LP was less then $25 \%$ of the harvested motion, while the fusion motion decreased greater then $75 \%$ in most cases.

The two-level fusion condition demonstrated significantly decreased motion at the operated levels and significantly increased motion adjacent to the operative levels as compared to harvested for normalized motion and MSU percent contribution. This was true in all cases except right axial rotation in MSU percent contribution at C4-C5, and left axial rotation in MSU percent contribution at C7-T1.

The current study biomechanically demonstrates a statistically significant increase of motion at the levels adjacent to simulated fusion in all loading cases, thereby indicating adjacent segment disease is a valid clinical concern in two-level fusion cases as well. As compared to two-level fusion disc arthroplasty induced a more physiological motion response at levels superior and inferior to the instrumented levels.

\subsection{Conclusion}

While ACDF procedures for treatment of cervical spine disease have very successful initial outcomes, it has been repeatedly shown that incidences of recurring surgery occur in as many as one out of four patients over the long-term. ${ }^{22}$ These complications introduce an opportunity for improvement in the treatment of end stage cervical disease. Arthroplasty is a widely accepted practice in the treatment of knee and hip degeneration and it is only natural that a spinal counterpart be the next step in degenerative diseases of the spine. This study has 
demonstrated the Prestige Low-Profile disc prosthesis presented in a cadaver model biomechanically favors natural cervical motion and may be an acceptable treatment option for degenerative cervical disc disease. 


\section{Biomechanical Comparison of Two-Level Arthroplasty Using the ProDisc-C with Two-Level Fusion}

\subsection{Introduction}

Degenerative disc diseases of the cervical spine are traditionally surgically treated with anterior cervical discectomy and fusion (ACDF). Approximately 225,000 fusion procedures were preformed in the United States in 2005. ${ }^{31}$ Although ACDF is the standard of care for degeneration of the cervical spine, an increasing number of clinical studies have shown a significant rate of adjacent segment disease and a reoccurrence of disc degeneration adjacent to a fusion site in patients treated with ACDF over the long term. Multi-level fusion has produced similar results. ${ }^{21,24,27}$ In addition McAfee et al. ${ }^{28}$ further reported that the complication rate increases with the number of fused levels.

Due to the development of long term ACDF complications, cervical disc arthroplasty has emerged with some success. An early clinical study by Wigfield et al. ${ }^{49}$ comparing disc arthroplasty with fusion found a significant difference in motion at the segments adjacent to the surgical site. Multi-level disc arthroplasty has also begun to emerge in the literature with positive clinical results. ${ }^{4,15,19,33,43}$ However, long term clinical data on patients with multi-level cervical disc arthroplasty has not yet be collected, and there are no biomechanical studies comparing two-level disc arthroplasty with two-level fusion in a human cadaver model. The objective of this study was to determine the biomechanical effects of adjacent two-level disc arthroplasty using the ProDisc-C disc prosthesis in an established cadaveric model. The twolevel arthroplasty model was compared to the harvested condition and a two-level fusion model. 


\subsection{Materials and Methods}

\subsubsection{Specimen Preparation and Spinal Instrumentation}

Eight fresh human cadaveric sub-axial cervical spines (mean age $71.3 \pm 9.2$ years) were wrapped in saline soaked gauze, placed in double plastic bags, and frozen at $-20^{\circ} \mathrm{C}$. Specimens consisted of seven intact vertebral bodies including second cervical vertebral body (C2) through the first thoracic vertebral body (T1). Prior to preparation, specimens were thawed for 12 hours in a standard refrigeration system. Study inclusion was determined using anterior-posterior and lateral radiographs, with exclusion criteria determined by existence of gross osteopenia or anatomic abnormality. Preparation for testing involved removal of excess cervical musculature, while preserving the spinal ligaments, discs, and bone. Additional tissue was removed at the $\mathrm{C} 2$ and $\mathrm{T} 1$ vertebral bodies (VB) to expose boney surfaces for potting material purchase. Screws were placed at the spinal processes, exposed facet joints, and exposed $\mathrm{C} 2$ and $\mathrm{T} 1$ vertebral bodies for additional potting material fixation within the potting material. The specimens were then mounted in molds and held with a positioning jig to maintain a level, upright, and neutral position with the anterior aspect of the facet joint estimated as the flexion/extension axis. Temporary screws passed through the sides of the molds and initially held the end bodies in place, while a low-melting-point bismuth alloy (Small Parts, Miami Lakes, FL) was allowed to solidify. This provided final fixation at the end bodies in the mounting pots. Threaded rods were inserted into the lateral aspects of the VB's for motion tracking target attachment and external fusion fixation. These rods did not interfere with device installation.

Three different spine conditions were evaluated: the non-instrumented condition (harvested), instrumented with two-level fusion (fusion) at levels C5-C6 and C6-C7, and prosthetic disc implantation at levels C5-C6 and C6-C7 (ProDisc-C, Synthes Spine, West 
Chester, PA). The ProDisc-C is a metal on ultra high molecular weight polymer articulating device with a theoretical instantaneous axis of rotation (IAR) located on the lower fixture where the ball is located. Keels on the device endplates provide initial fixation as shown in Figure 4-1. Fusion was simulated using a customized external fixation system. The three spine conditions are shown in Figure 4-2.

\subsubsection{Non-Destructive Biomechanical Testing Protocol}

All spines were non-destructively tested in flexion, extension, lateral bending, and axial rotation. All tests were performed under displacement control. The specific displacement control testing protocol is described elsewhere ${ }^{8,12}$ and has been used in other cervical spine studies to evaluate fusion techniques ${ }^{14,17,40}$ as well as other prosthetic disc devices. ${ }^{13,14}$ Due to the strong coupling between physiological axial rotation and lateral bending ${ }^{36,47}$ axial rotation was unconstrained in lateral bending testing and lateral bending was unconstrained during axial rotation tests.

For flexion, extension, and lateral bending tests, a compressive load was applied at a 200 $\mathrm{mm}$ offset from the disc midline at a constant actuator velocity of $6.4 \mathrm{~mm} / \mathrm{sec}$ approximately 2 $\mathrm{deg} / \mathrm{sec}$ of global rotation) until either a global moment of $3.5 \mathrm{Nm}$ or a global rotation of 45 degrees was satisfied. Axial rotation end limits included a global moment of $3.5 \mathrm{Nm}$ or a global rotation of 45 degrees at a rate of $2.25 \mathrm{deg} / \mathrm{sec}$. All specimens were pre-conditioned with at least five cycles prior to the final trial of three consecutive cycles. Specimens were regularly moistened with a $0.9 \%$ saline mist throughout preparation and testing. 


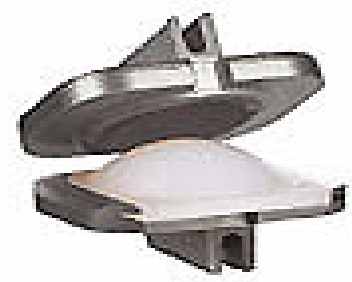

Figure 4-1: ProDisc-C Disc Prosthesis. (Used with permission from Synthes Spine.)

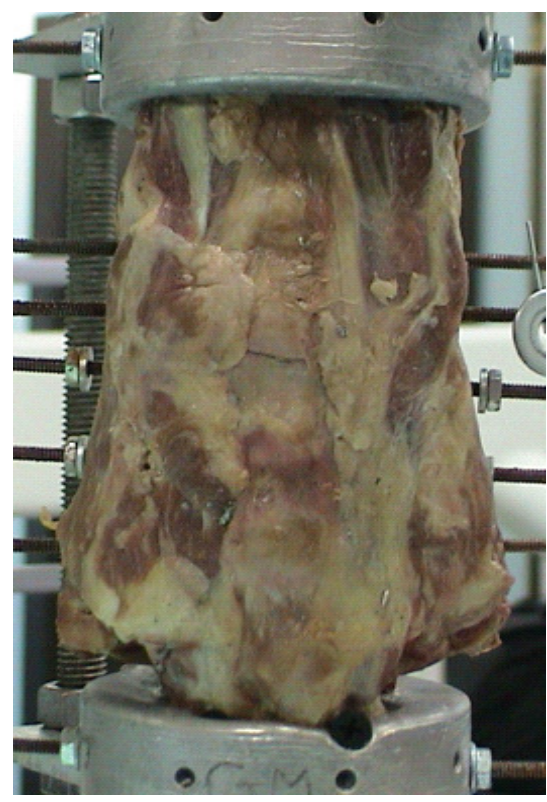

a

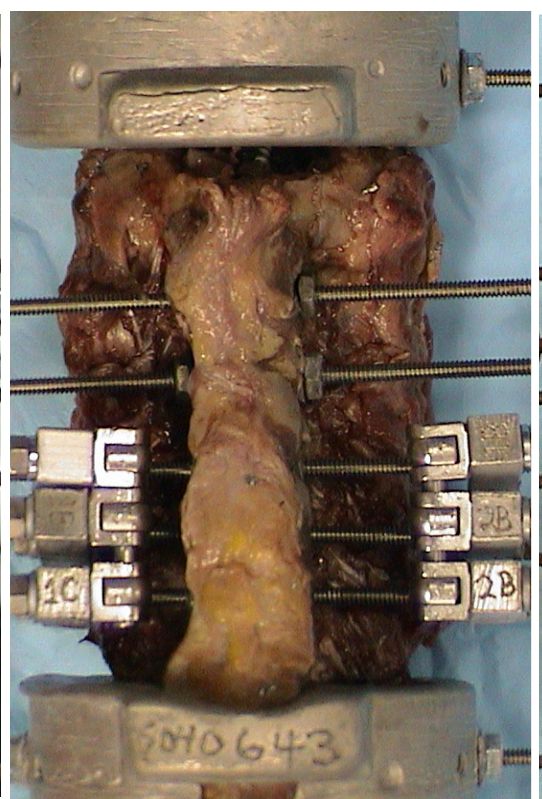

b

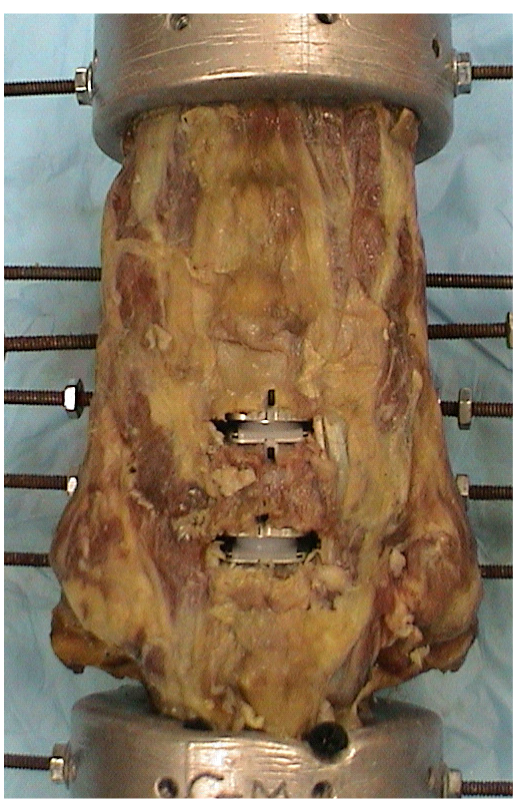

c

Figure 4-2: Specimen Conditions. (a) Harvested spine. (b) Spine instrumented with two-level fusion at levels $\mathrm{C} 5$ through C7. (c) Specimen instrumented with two-level disc arthroplasty using the ProDisc-C at cervical levels C5-C6 and C6-C7. 


\subsubsection{Data Management and Statistical Analysis}

Load and displacement measurements were acquired with a dedicated analog to digital (A/D) data acquisition system and sampled at $10 \mathrm{~Hz}$. A three dimensional non-contact measurement system was used to track segmental cervical motion for each testing condition. Global rotation and applied moment data were combined to calculate the overall spine flexibility and compared at a common end limit of applied moment within each spine condition.

Instrumented spine conditions were normalized with respect to their harvested condition before comparison to account for intrinsic differences in specimen variability. Motion response at the operated regions were compared by determining the relative contribution of levels C5-C7 to the overall global motion, and then normalizing with respect to the intact spine condition. The same normalization procedure was used to compare percent changes in individual MSU rotations at the remaining non-operated spinal levels for the instrumented spine conditions. MSU percent change in contribution compares the percent changes in individual MSU rotations at the remaining non-operated spinal levels for the instrumented spine conditions. MSU percent change in contribution compares the percent motion contribution of each operative condition at a particular MSU to the percent motion contribution of the harvested condition at that same MSU. The percent motion contribution is the motion contribution at a particular MSU divided by the total global motion of the specimen expressed as a percentage.

A statistically significant difference among the three groups was detected using one-way analysis of variance (ANOVA). Significantly different values detected with ANOVA were further analyzed for pair wise comparison using the Student Newman Keuls (SNK) test. Significance was defined as $\mathrm{P}<0.05$. Normality and equal variance was assumed for all groups in both ANOVA and SNK analysis. 


\subsection{Results}

\subsubsection{Normalized Flexibility}

The global flexibility values for the implanted and fused spine conditions are shown in Table 4-1. The global flexibility values of the instrumented conditions were normalized to the harvested condition and compared at the largest amount of applied moment common to all spine conditions and are given in Table 4-2. The disc arthroplasty condition was not significantly different from the harvested condition, except in extension and right axial rotation. Fusion significantly decreased flexibility relative to the harvested condition in flexion and extension. The fusion and ProDisc-C spine conditions were significantly different in flexion, extension, and right axial rotation.

\subsubsection{Normalized Motion}

The mean relative MSU rotations of the treated levels (C5-C7) are shown in Table 4-3 for all three spine conditions. The contribution of motion at the treated levels relative to the total spine rotation for the fused and implanted levels were normalized to the harvested condition and are shown in Figures 4-3, 4-4, 4-5. A normalized value equal to one is equivalent to $100 \%$ of the harvested condition. The two-level ProDisc-C condition was not significantly different from the harvested condition except in flexion $(117 \%$ of $\mathrm{H})$ and left axial rotation $(122 \%$ of $\mathrm{H})$. The normalized motion for the fused condition was significantly lower for both the harvested and disc implant condition for all testing modes. 
Table 4-1: Global Rotation Versus Applied Moment Data for ProDisc-C.

\begin{tabular}{ccccc}
\hline \multirow{2}{*}{ Test Mode } & $\begin{array}{c}\text { Spine } \\
\text { Condition }\end{array}$ & $\begin{array}{c}\text { Global } \\
\text { Moment }(\mathbf{N m})\end{array}$ & $\begin{array}{c}\text { Global Rotation } \\
(\mathbf{D e g})\end{array}$ & $\begin{array}{c}\text { Relative Flexibility } \\
\text { (Deg/Nm) }\end{array}$ \\
\hline \multirow{3}{*}{ Flexion } & Harvested & 3.4 & $35.6 \pm 7.7$ & $10.8 \pm 4.5$ \\
& Prestige-LP & 3.4 & $32.6 \pm 7.9$ & $10.0 \pm 4.2$ \\
& Fusion & 3.4 & $27.9 \pm 7.6$ & $8.6 \pm 4.2$ \\
\hline \multirow{3}{*}{ Extension } & Harvested & 1.6 & $29.7 \pm 5.1$ & $31.4 \pm 22.5$ \\
& Prestige-LP & 1.6 & $38.2 \pm 6.3$ & $37.8 \pm 23.4$ \\
& Fusion & 1.6 & $19.0 \pm 8.9$ & $16.5 \pm 13.5$ \\
\hline \multirow{3}{*}{ Left Lateral } & Harvested & 3.5 & $29.3 \pm 8.4$ & $8.1 \pm 2.3$ \\
& Prestige-LP & 3.5 & $29.9 \pm 5.9$ & $8.4 \pm 1.6$ \\
& Fusion & 3.5 & $28.5 \pm 9.1$ & $7.9 \pm 2.5$ \\
\hline \multirow{3}{*}{ Right Lateral } & Harvested & 3.5 & $28.9 \pm 7.1$ & $8.1 \pm 2.0$ \\
& Prestige-LP & 3.5 & $30.9 \pm 5.4$ & $8.5 \pm 1.5$ \\
& Fusion & 3.5 & $28.6 \pm 5.3$ & $8.0 \pm 1.5$ \\
\hline \multirow{3}{*}{ Left Axial } & Harvested & 3.5 & $23.1 \pm 6.5$ & $6.7 \pm 2.3$ \\
& Prestige-LP & 3.5 & $25.2 \pm 6.6$ & $7.2 \pm 1.8$ \\
& Fusion & 3.5 & $21.5 \pm 6.4$ & $6.3 \pm 2.4$ \\
\hline \multirow{2}{*}{ Right Axial } & Harvested & 3.3 & $18.8 \pm 6.3$ & $5.9 \pm 2.7$ \\
& Prestige-LP & 3.3 & $24.0 \pm 5.4$ & $7.6 \pm 3.3$ \\
& Fusion & 3.1 & $18.6 \pm 7.0$ & $5.7 \pm 2.2$ \\
\hline
\end{tabular}

Table 4-2: Normalized Flexibility for ProDisc-C Study.

\begin{tabular}{lcc}
\hline Test & Normalized ProDisc-C/Harvested & Normalized Fusion/Harvested \\
\hline Flexion & $0.93 \pm 0.13 \#$ & $0.79 \pm 0.08 * \#$ \\
Extension & $1.32 \pm 0.27 * \#$ & $0.63 \pm 0.29 * \#$ \\
Left Lateral & $1.06 \pm 0.17$ & $0.97 \pm 0.08$ \\
Right Lateral & $1.11 \pm 0.29$ & $1.02 \pm 0.24$ \\
Left Axial & $1.10 \pm 0.18$ & $0.95 \pm 0.15$ \\
Right Axial & $1.34 \pm 0.17 * \#$ & $1.00 \pm 0.14 \quad \#$ \\
\hline
\end{tabular}

* Signifies significant difference from the harvested condition.

\# Signifies significant difference between ProDisc-C and fusion. 
Table 4-3: Mean Relative Motion.

\begin{tabular}{|c|c|c|c|c|c|}
\hline Test Mode & $\begin{array}{c}\text { Spine } \\
\text { Condition }\end{array}$ & $\begin{array}{c}\text { Global } \\
\text { Motion } \\
\text { (Deg) }\end{array}$ & $\begin{array}{c}\text { Operative } \\
\text { Motion } \\
\text { (Deg) }\end{array}$ & $\begin{array}{l}\text { Relative } \\
\text { Motion }\end{array}$ & $\begin{array}{c}\text { Normalized } \\
\text { Motion }\end{array}$ \\
\hline \multirow{3}{*}{ Flexion } & Harvested & $35.6 \pm 7.7$ & $14.8 \pm 3.4$ & $0.42 \pm 0.04$ & 1.0 \\
\hline & ProDisc-C & $32.6 \pm 7.9$ & $16.0 \pm 5.4$ & $0.48 \pm 0.07$ & $1.17 \pm 0.18 * \#$ \\
\hline & Fusion & $27.9 \pm 7.6$ & $0.3 \pm 0.1$ & $0.01 \pm 0.01$ & $0.03 \pm 0.01 * \#$ \\
\hline \multirow{3}{*}{ Extension } & Harvested & $29.7 \pm 5.1$ & $12.2 \pm 4.3$ & $0.40 \pm 0.08$ & 1.0 \\
\hline & ProDisc-C & $38.2 \pm 6.3$ & $14.3 \pm 6.0$ & $0.36 \pm 0.13$ & $0.90 \pm 0.32 \quad \#$ \\
\hline & Fusion & $19.0 \pm 8.9$ & $0.2 \pm 0.2$ & $0.03 \pm 0.03$ & $0.08 \pm 0.07 * \#$ \\
\hline \multirow{3}{*}{$\begin{array}{c}\text { Flexion + } \\
\text { Extension }\end{array}$} & Harvested & $65.3 \pm 11.7$ & $27.0 \pm 6.9$ & $0.41 \pm 0.04$ & 1.0 \\
\hline & ProDisc-C & $70.8 \pm 11.0$ & $30.3 \pm 8.0$ & $0.42 \pm 0.06$ & $1.03 \pm 0.15$ \\
\hline & Fusion & $46.9 \pm 12.8$ & $0.7 \pm 0.1$ & $0.02 \pm 0.01$ & $0.04 \pm 0.01 * \#$ \\
\hline \multirow{3}{*}{ Left Lateral } & Harvested & $29.3 \pm 8.4$ & $10.2 \pm 4.1$ & $0.34 \pm 0.06$ & 1.0 \\
\hline & ProDisc-C & $29.9 \pm 5.9$ & $10.4 \pm 3.9$ & $0.34 \pm 0.09$ & $1.01 \pm 0.20$ \\
\hline & Fusion & $28.5 \pm 9.1$ & $0.9 \pm 1.4$ & $0.03 \pm 0.03$ & $0.07 \pm 0.07 * \#$ \\
\hline \multirow{3}{*}{$\begin{array}{l}\text { Right } \\
\text { Lateral }\end{array}$} & Harvested & $28.9 \pm 7.1$ & $10.3 \pm 3.3$ & $0.35 \pm 0.04$ & 1.0 \\
\hline & ProDisc-C & $28.9 \pm 7.2$ & $9.3 \pm 2.7$ & $0.33 \pm 0.07$ & $0.94 \pm 0.23$ \\
\hline & Fusion & $27.4 \pm 6.1$ & $0.8 \pm 0.4$ & $0.03 \pm 0.01$ & $0.09 \pm 0.05 * \#$ \\
\hline \multirow{3}{*}{$\begin{array}{c}\text { Left + Right } \\
\text { Lateral }\end{array}$} & Harvested & $58.2 \pm 15.0$ & $20.5 \pm 7.2$ & $0.35 \pm 0.05$ & 1.0 \\
\hline & ProDisc-C & $58.7 \pm 12.3$ & $19.7 \pm 6.0$ & $0.33 \pm 0.07$ & $0.97 \pm 0.19$ \\
\hline & Fusion & $55.9 \pm 14.9$ & $1.7 \pm 1.6$ & $0.03 \pm 0.02$ & $0.08 \pm 0.04 * \#$ \\
\hline \multirow{3}{*}{ Left Axial } & Harvested & $23.1 \pm 6.5$ & $6.1 \pm 2.4$ & $0.27 \pm 0.09$ & 1.0 \\
\hline & ProDisc-C & $25.2 \pm 6.6$ & $7.7 \pm 2.7$ & $0.31 \pm 0.08$ & $1.22 \pm 0.24 * \#$ \\
\hline & Fusion & $21.5 \pm 6.4$ & $0.7 \pm 0.3$ & $0.04 \pm 0.02$ & $0.14 \pm 0.08 * \#$ \\
\hline \multirow{3}{*}{ Right Axial } & Harvested & $18.8 \pm 6.4$ & $6.1 \pm 2.7$ & $0.32 \pm 0.08$ & 1.0 \\
\hline & ProDisc-C & $24.0 \pm 5.4$ & $8.9 \pm 3.3$ & $0.38 \pm 0.12$ & $1.24 \pm 0.56$ \\
\hline & Fusion & $18.5 \pm 7.0$ & $1.0 \pm 0.9$ & $0.06 \pm 0.07$ & $0.22 \pm 0.24 * \#$ \\
\hline \multirow{3}{*}{$\begin{array}{c}\text { Left }+ \text { Right } \\
\text { Axial }\end{array}$} & Harvested & $41.9 \pm 12.0$ & $12.2 \pm 4.8$ & $0.29 \pm 0.08$ & 1.0 \\
\hline & ProDisc-C & $49.2 \pm 10.5$ & $16.6 \pm 5.2$ & $0.33 \pm 0.05$ & $1.23 \pm 0.37$ \\
\hline & Fusion & $40.1 \pm 9.0$ & $1.8 \pm 1.0$ & $0.05 \pm 0.04$ & $0.17 \pm 0.13 * \#$ \\
\hline
\end{tabular}

* Signifies significant difference from the harvested condition. \# Signifies significant difference between ProDisc-C and fusion. 


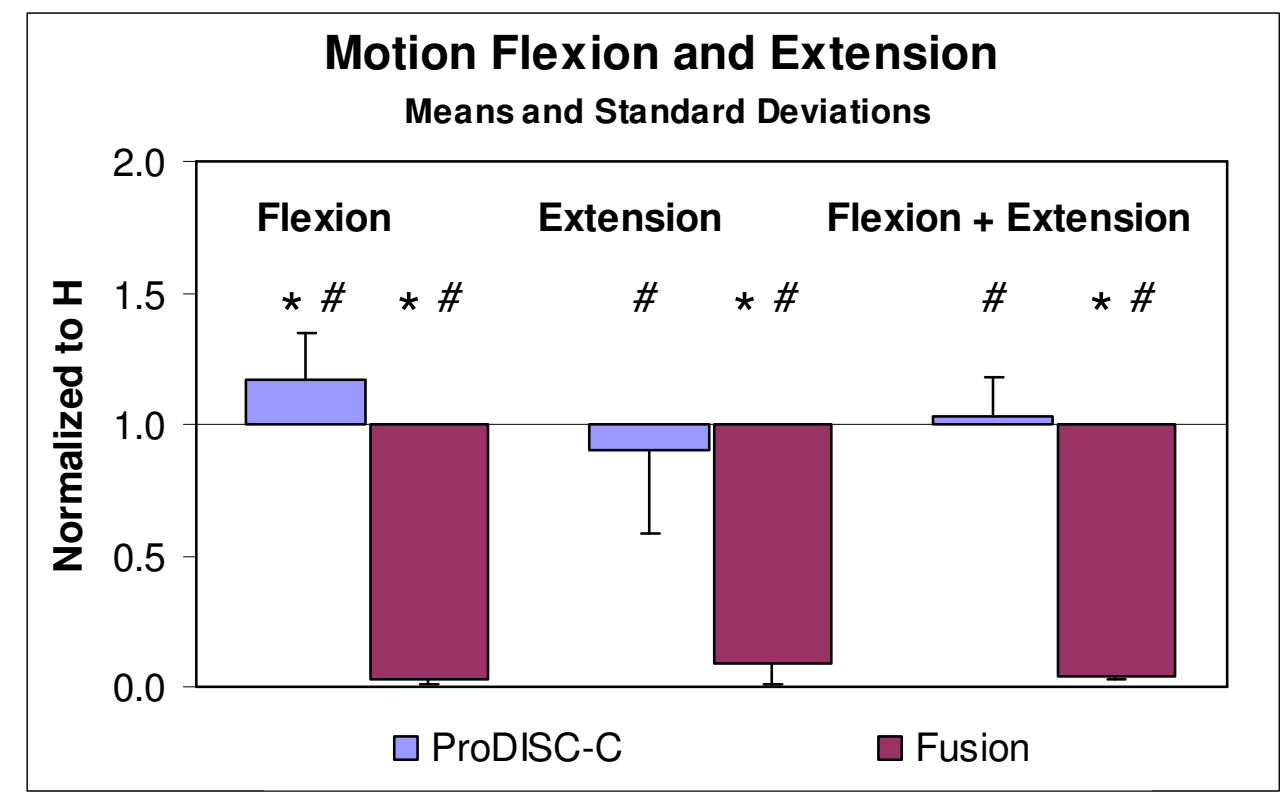

Figure 4-3: ProDisc-C Normalized Motion for Flexion and Extension. * Signifies significant difference from the harvested $(\mathrm{H})$ condition. \# Signifies significant difference between ProDisc-C and fusion.

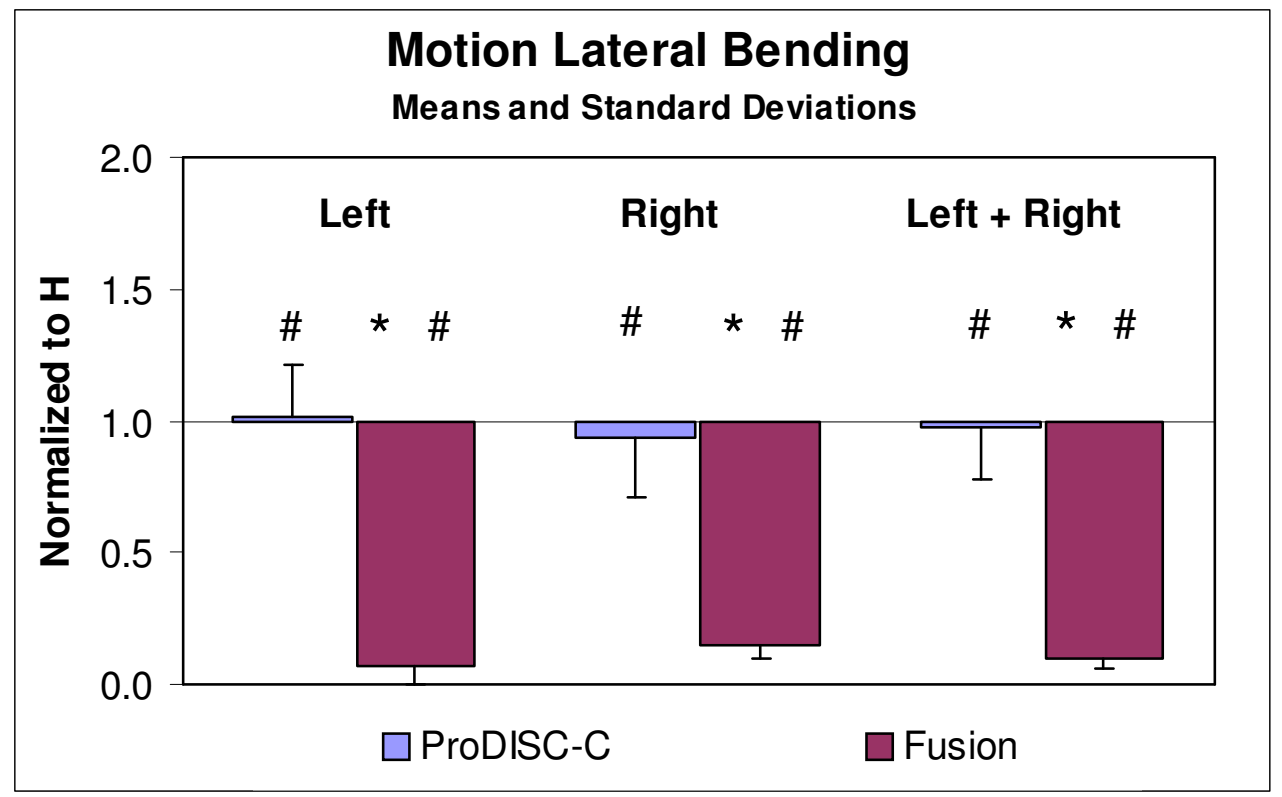

Figure 4-4: ProDisc-C Normalized Motion for Lateral Bending. * Signifies significant difference from harvested $(\mathrm{H})$. \# Signifies significant difference between ProDisc-C and fusion. 


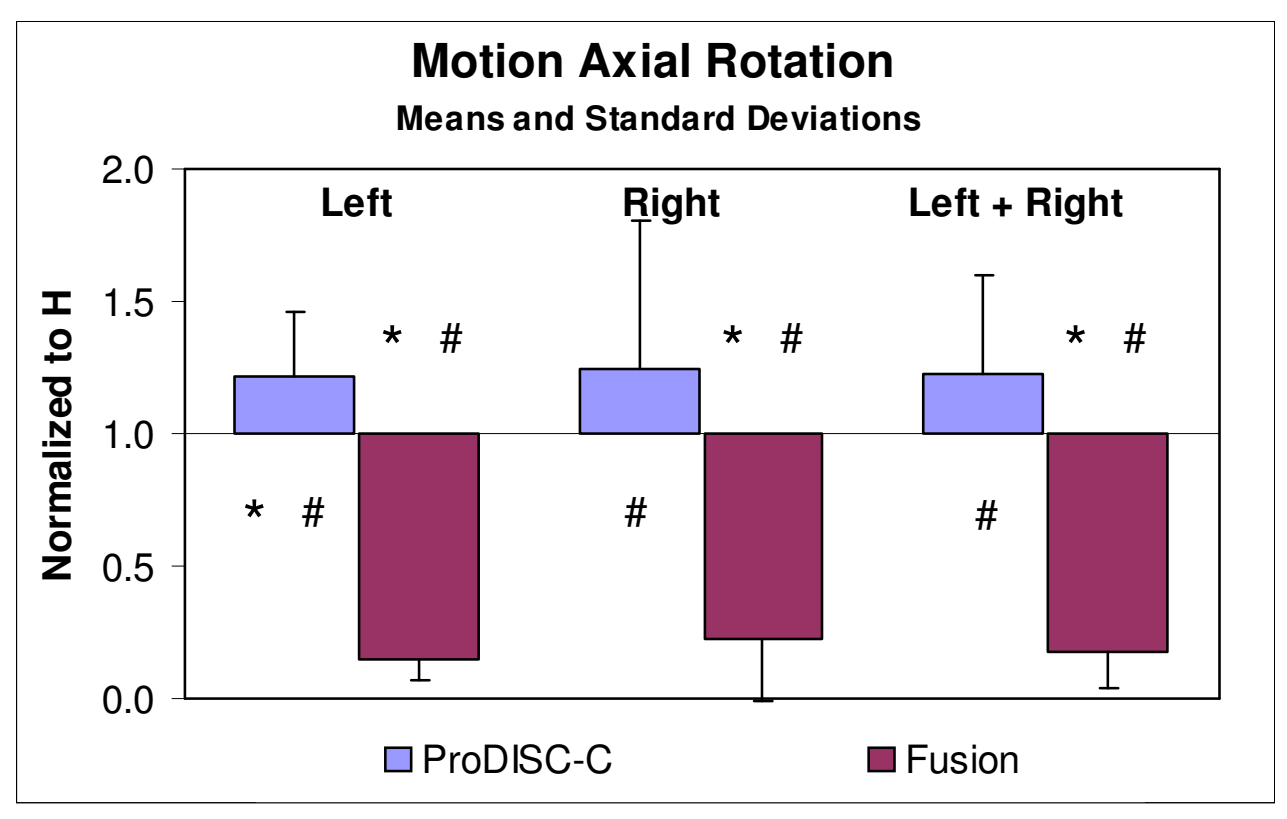

Figure 4-5: ProDisc-C Normalized Motion for Axial Rotation. * Signifies significant difference relative to the harvested $(\mathrm{H})$ condition. \# Signifies significant difference between ProDisc-C and fusion spine condition.

\subsubsection{Changes in MSU Contribution}

The relative MSU rotations for the harvested, two-level fused, and two-level ProDisc-C spine conditions during flexion-extension, left and right lateral bending, and left and right axial rotation are shown in Figure 4-6. The greatest range of flexion and extension occurred at C5-C6, followed by C4-C5, and C6-C7 spinal levels. The changes in the MSU rotation contribution at each level, expressed as a percentage of the overall global (C2-T1) rotation for the ProDisc-C and fused conditions were normalized to its corresponding contribution in the harvested condition. For example, at C5-C6 the percent change in contribution would be the ratio of ([(C5C6) rotation of instrumented spine $] /[(\mathrm{C} 2-\mathrm{T} 1)$ rotation of instrumented spine] divided by [(C5-C6) rotation of harvested spine $] /[(\mathrm{C} 2-\mathrm{T} 1)$ rotation of harvested spine $])$. The MSU percent change in 

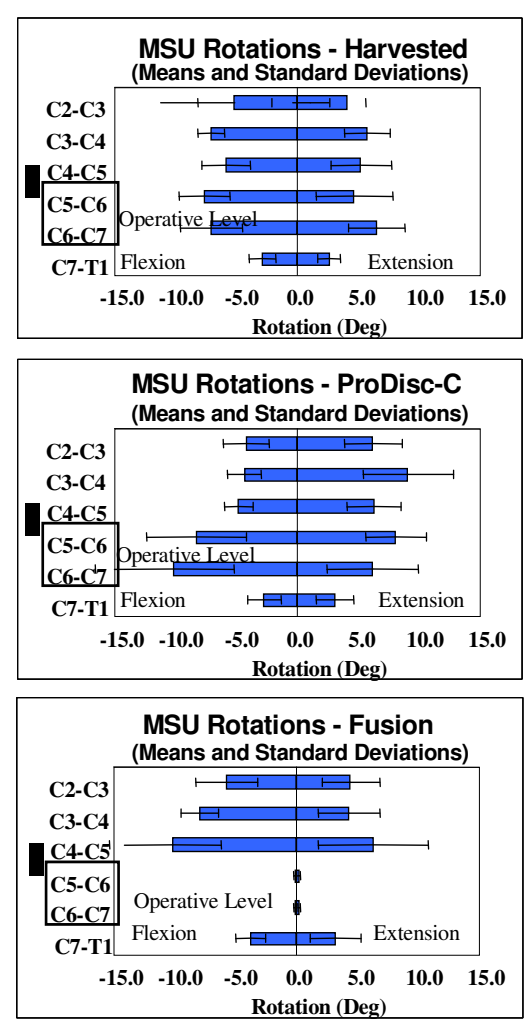
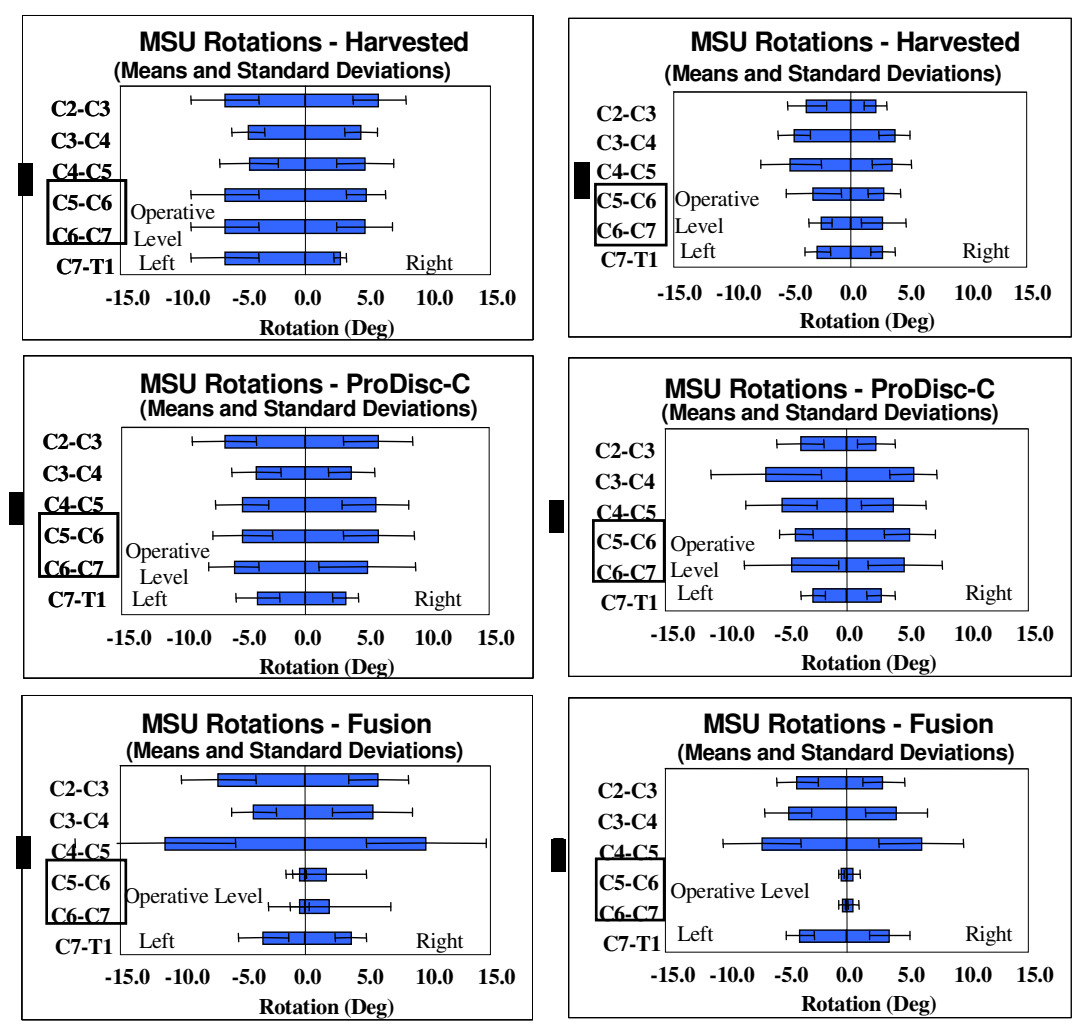

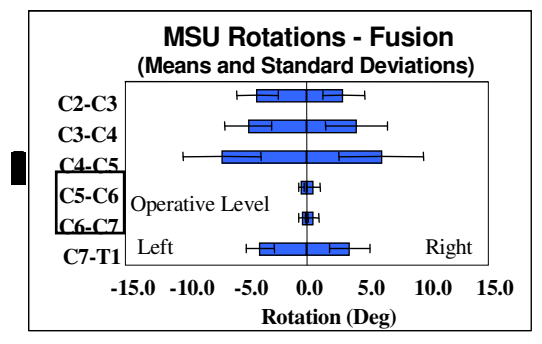

Figure 4-6: ProDisc-C MSU Rotations. Columns represent the testing mode: flexion/extension, lateral bending, and axial rotation. Rows represent the spinal condition: harvested, ProDisc-C, and fusion. 
flexion-extension, lateral bending, and axial rotation are shown in Figures 4-7, 4-8, and 4-9 respectively.

Two-level ProDisc-C arthroplasty was not significantly different from the harvested spine at the implanted levels (C5-C6 and C6-C7) except in flexion and extension at C6-C7. Two-level fusion was significantly different from two-level ProDisc-C arthroplasty at the operated levels in all testing modes except at C6-C7 during right lateral bending. Two-level fusion significantly decreased motion compared to the harvested spine at the operative levels $\mathrm{C} 5-\mathrm{C} 6$ and $\mathrm{C} 6-\mathrm{C} 7$ for all loading cases.

The levels superior (C4-C5) and inferior (C7-T1) to the fused levels both showed a statistically significant increase in motion relative to harvested spines for all loading cases except left and right lateral bending at $\mathrm{C} 4-\mathrm{C} 5$, and in right axial rotation at $\mathrm{C} 7-\mathrm{T} 1$. No significant differences occurred between the ProDisc-C and harvested spines for any loading case at the immediately adjacent levels. Fusion was significantly different from ProDisc-C at C4-C5 and C7-T1 in all loading conditions, except in left and right lateral bending where only C4-C5 showed differences and left axial rotation at C4-C5.

\subsection{Discussion}

Cervical disc replacement is indicated for symptomatic relief of neurological discomfort through decompression of the nerve root while retaining motion at the effected MSU. ${ }^{25,46}$ The common idea that maintenance of motion at the cervical joint may result in improved load and a reduction of stress on adjacent segments, thereby reducing the potential for painful adjacent

disease to occur, has been a proposed advantage of cervical arthroplasty. ${ }^{24,25,32,33,46}$ 

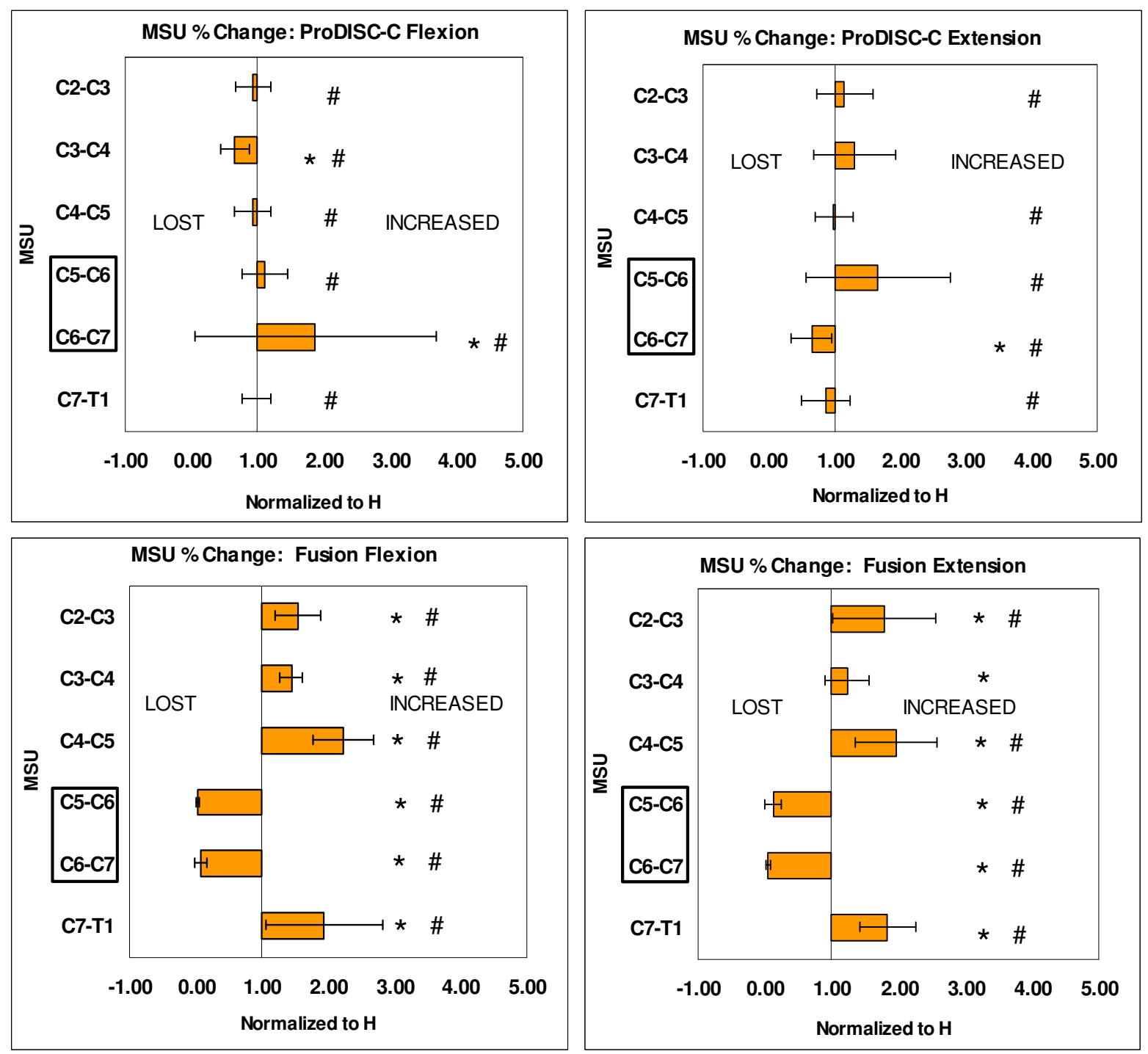

$\mathbf{a}$

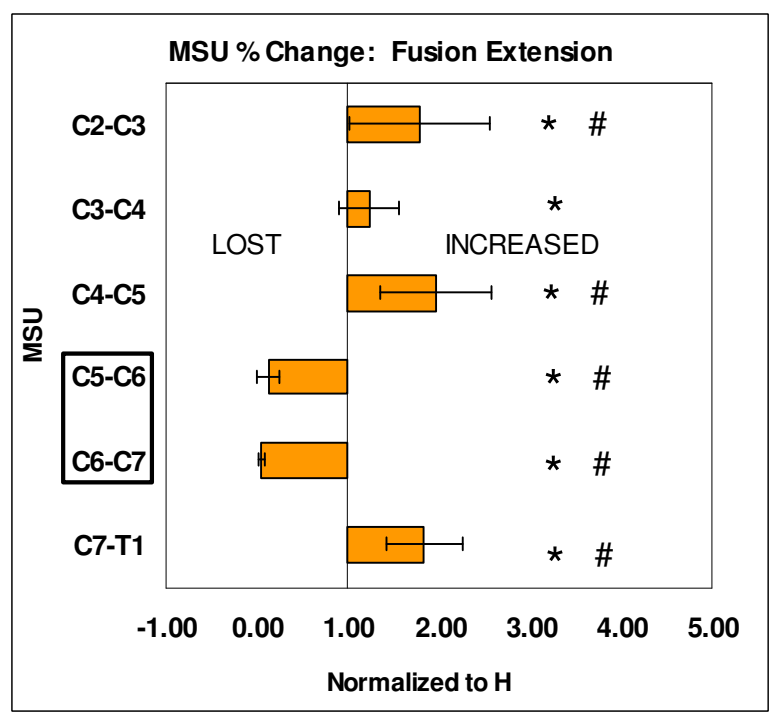

b

Figure 4-7: ProDisc-C MSU Percent Change. (a) Flexion and (b) extension. All values are relative to the harvested condition, represented by unity. * Signifies significant difference from harvested $(\mathrm{H})$. \# Signifies significant difference between ProDisc-C and fusion. 

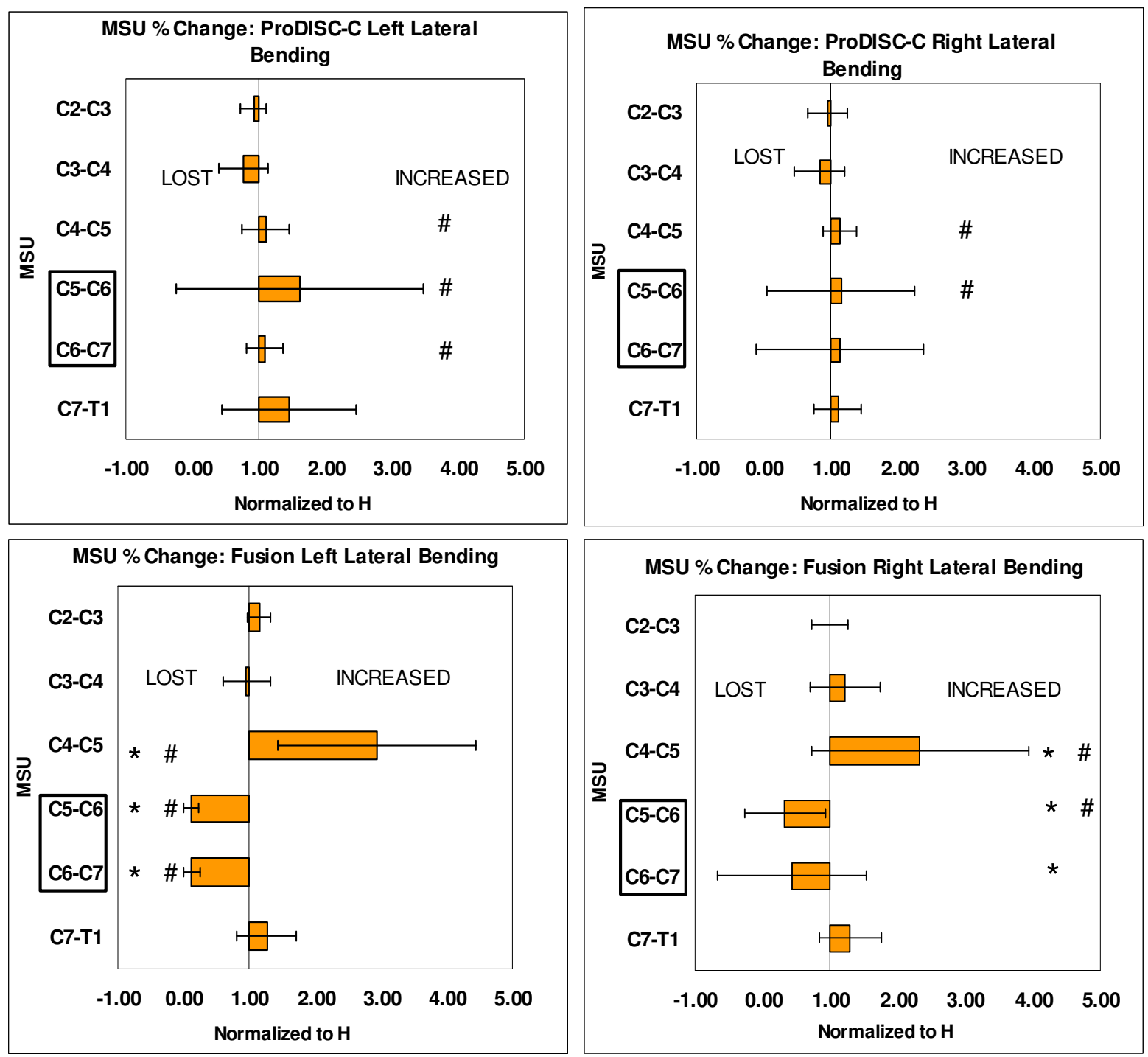

a

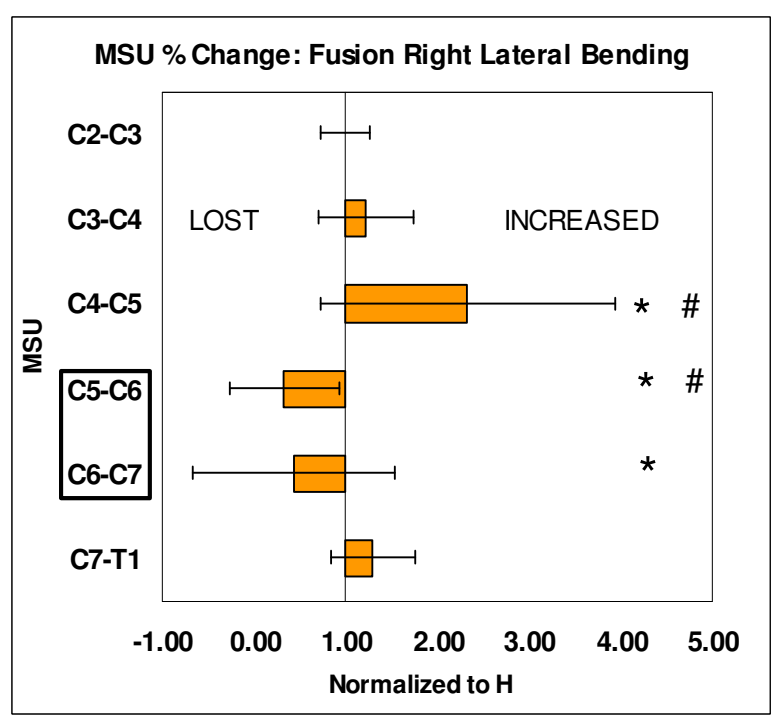

b

Figure 4-8: ProDisc-C MSU Percent Change in Lateral Bending. (a) Left and (b) right lateral bending. All values are relative to the harvested condition, represented by unity. * Signifies significant difference from harvested $(\mathrm{H})$. \# Signifies significant difference between ProDisc-C and fusion. 

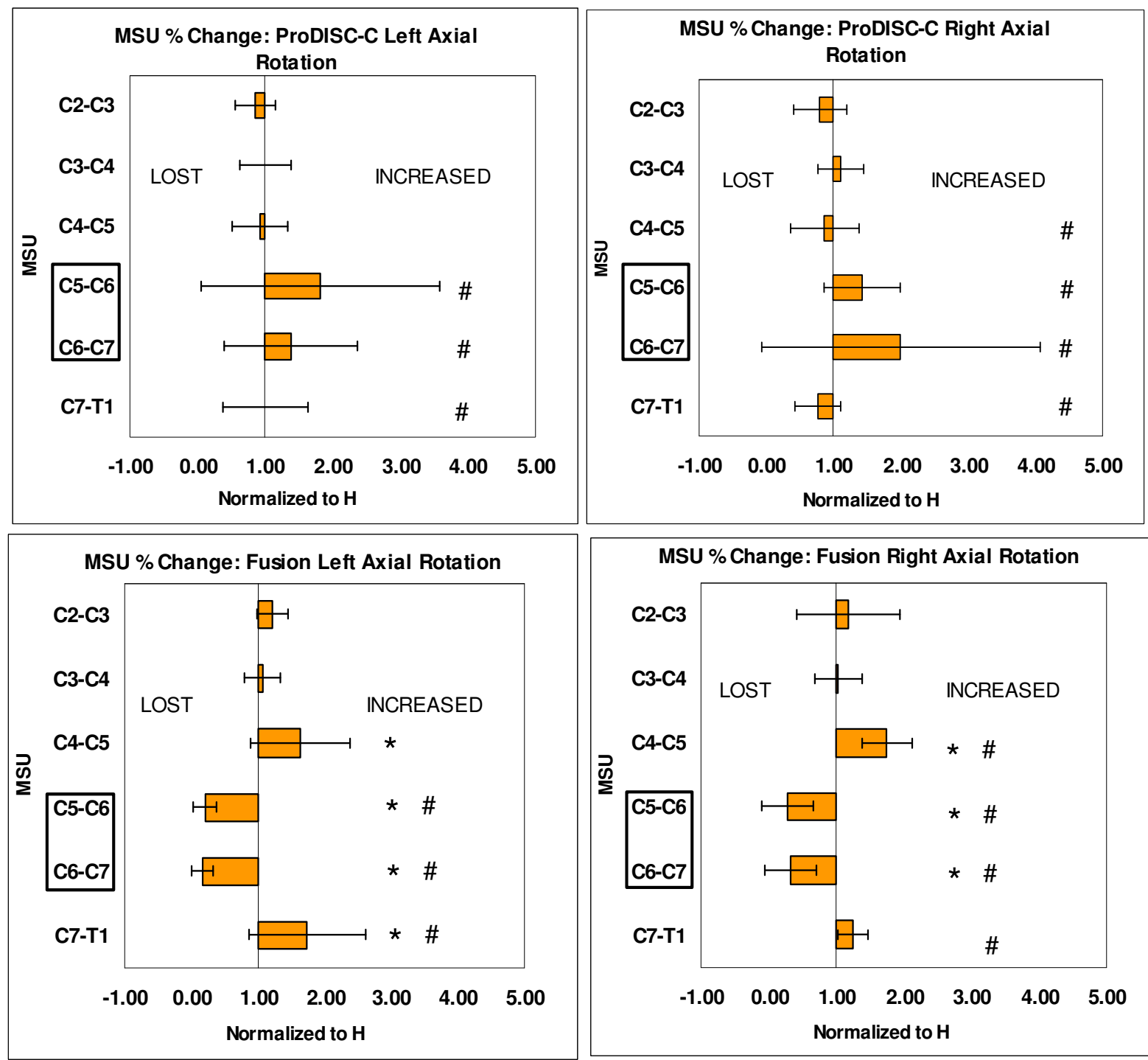

$\mathbf{a}$

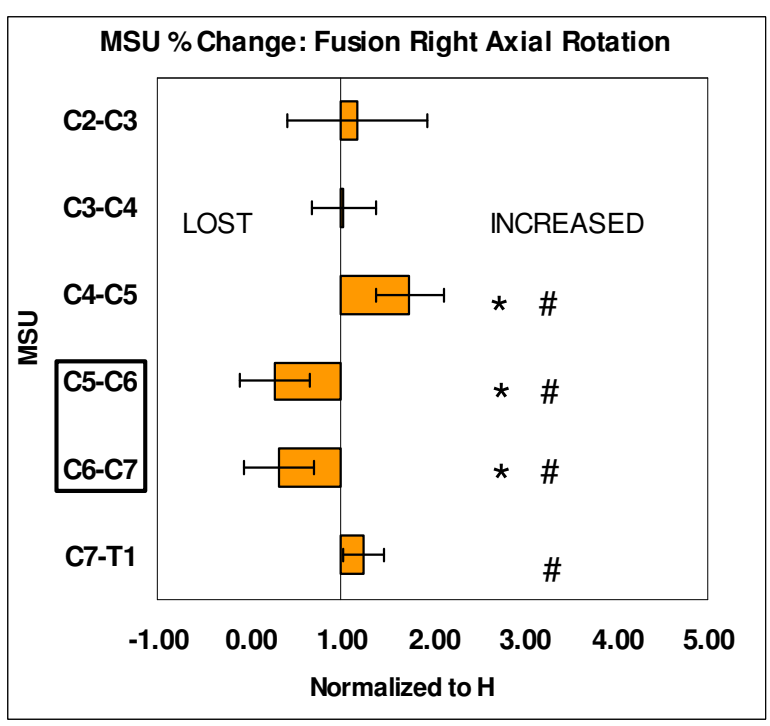

b

Figure 4-9: ProDisc-C MSU Percent Change in Axial Rotation. (a) Left and (b) right axial rotation. All values are relative to the harvested $(\mathrm{H})$ condition, represented by unity. * Signifies significant difference from harvested. \# Signifies significant difference between ProDisc-C and fusion. 
Robertson et al. ${ }^{38}$ compared adjacent segment outcomes between patients receiving a single level cervical fusion using the Affinity Anterior Cervical Cage System or single level cervical arthroplasty using the Bryan Artificial Cervical Disc in a two year follow-up transfer study. The results indicated a significant difference in the occurrence of symptomatic adjacent segment disease between the fusion and arthroplasty group. Those who received fusion were also significantly more likely to require additional medical treatment attributing to new adjacent disease. Wingfield et al. ${ }^{48}$ studied early clinical results of cervical arthrodesis versus arthroplasty in 15 patients. They found an increase in motion at the adjacent levels after the fusion procedure, which increased by $5 \%$ at 6 months and $15 \%$ at 12 months, and maintenance of motion at the arthroplasty site.

Other clinicians have found similar symptomatic results at adjacent levels in studies evaluating two-level fusion. Gore and Sepic ${ }^{21}$ evaluated 17 patients receiving two-level fusion throughout an average 21-year follow up and found $18 \%$ of these patients required additional procedures at adjacent segments. Hilibrand et al. ${ }^{22}$ estimated that $25 \%$ of patients receiving ACDF would develop adjacent segment disease within 10 years.

Clinically there may be instances to treat a level adjacent to an existing disc implantation. In a previous biomechanical study evaluating single level ProDisc-C disc implantation a human single level cadaveric model, global flexibility data produced results similar to this study. ${ }^{13} \mathrm{~A}$ significant difference in the flexibility data occurred between the harvested and single level ProDisc-C spine conditions in extension and right axial rotation, as in the current study. The single level ProDisc-C was also significantly different from fusion in right axial rotation. This similarity may imply that implanting a second disc implant at the inferior level adjacent to an existing disc does not compromise flexibility of the sub-axial cervical spine. 
Further, when analyzing the motion response at the two treated MSU levels (C5-C6 and C6-C7) relative to the total (C2-T1) spinal rotation, the single level ProDisc-C condition was only different from the harvested spine condition in flexion $(153 \%$ of $\mathrm{H})$, as shown in Figure 4-10. In the two-level ProDisc-C study, the only significant differences between the ProDisc-C and harvested spine conditions occurred in flexion $(117 \%$ of $\mathrm{H})$ and left axial rotation $(122 \%$ of $\mathrm{H})$. In both studies the deviations from the harvested condition were less than $25 \%$ of the intact value. It would appear as though addition of a disc prosthesis adjacent to an existing implant does not alter the motion response at the second treated level and may be a viable alternative to fusion surgery.

As observed with other fusion studies, two-level fusion caused a significant decrease in motion at the treated levels and a significant increase in motion adjacent to the operative site compared to harvested spine condition. A significant increase in motion at levels adjacent to the

operative level may be clinically observed as adjacent segment disease. ${ }^{3,9,20,24,32}$ However, use of two-level ProDisc-C devices retained physiological motion at levels superior and inferior to the instrumented levels, which may help to minimize the advancement of adjacent segment disease.

\subsection{Conclusion}

Two-level cervical arthroplasty with the ProDisc-C disc produced spinal motion patterns that were comparable to the harvested spine at both the treated and adjacent levels. Simulated fusion, on the other hand, markedly reduced motion at the operated levels and produced a compensatory increase in motion at adjacent segments. From a biomechanical point of view, 


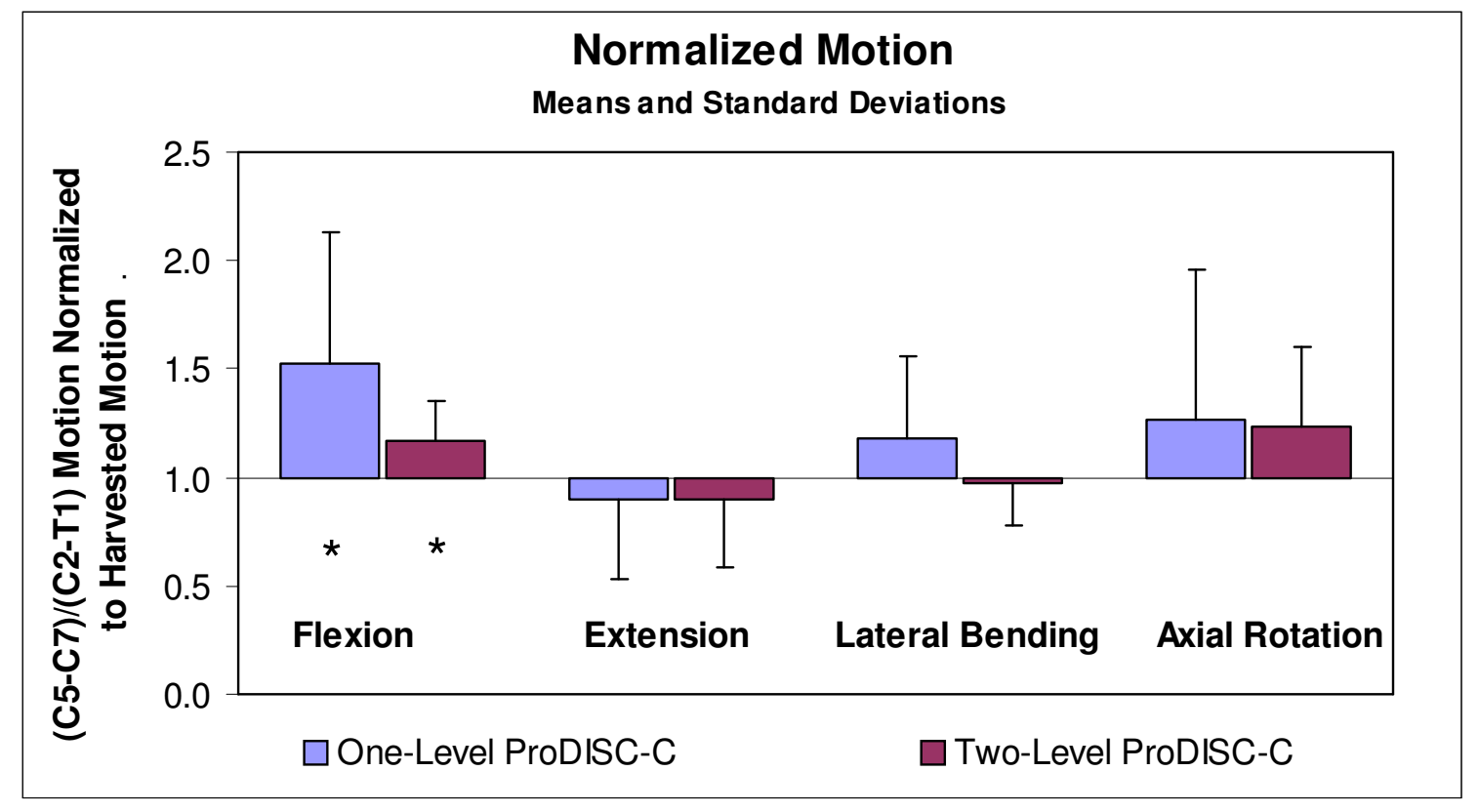

Figure 4-10: Normalized Motion Comparing One-Level and Two-Level ProDisc-C. (C5-C7) MSU rotation relative to the total (C2-T1) rotation for implanted condition normalized to respective harvested contribution. * Signifies a significant difference between one-level ProDisc-C and two-level ProDisc-C. 
two-level cervical arthroplasty yielded a spine that was much closer to the baseline (unoperated) state than did fusion. 


\section{Multi-Level Disc Arthroplasty: A Comparison of Two Prosthetic Disc Devices}

\subsection{Introduction}

According to the current U.S. National Health Survey, $14.7 \%$ of adults over 18 years of age experience neck pain lasting at least one full day. ${ }^{7}$ Anterior cervical discectomy and fusion $(\mathrm{ACDF})$ procedures are the most common surgical treatment for cervical spine degenerative diseases. In fact, approximately 237,800 anterior cervical spine procedures were projected in 2005 with this number projected to increase by $8.0 \%$ annually over the next few years. ${ }^{23}$ It has been widely documented that adjacent segment disease, a reoccurrence of degenerative disc disease above or below the operated level, is common when ACDF is performed. 3, 5, 9, 20, 22, 24, 27,

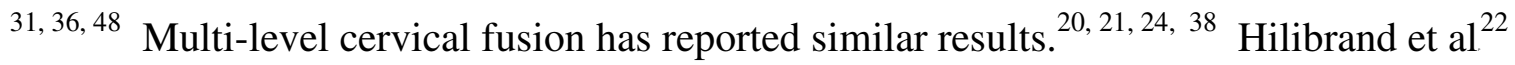
determined symptomatic adjacent segment disease potentially affects $25 \%$ of patients within ten years of anterior cervical arthrodesis. However, this was found to be significantly greater in subjects with one-level arthrodesis compared to two-level arthrodesis. Gore and Sepic ${ }^{21}$ presented 17 patients with two-level anterior discectomy and fusion in a 21-year average follow up study. Eighteen percent of these patients experienced reoccurring pain, which required further surgery to correct for adjacent segment degeneration. A statistically significant increase of motion at levels adjacent to simulated fusion in all loading cases occurred in this study, thereby suggesting adjacent segment disease is a valid clinical concern in two-level fusion cases as well. 
Disc replacement surgery using a cervical disc prosthesis may be an alternative treatment aimed at restoring spinal motion, which may prevent adjacent segment disease otherwise found with fusion. In fact, an early clinical study comparing fusion and prosthetic disc patient populations showed there was a significant difference in motion at adjacent segments between the two groups. ${ }^{49}$ Two-level artificial disc implantation has begun to surface in the clinical literature with some positive results, ${ }^{11,15,19,28,33,43}$ however few biomechanical studies of this nature have been documented. In addition, a majority of the clinical studies including multilevel arthroplasty patients do not analyze them separately from single-level disc arthroplasty patients. The objective of this study was to assess the ability of multi-level disc prostheses to restore cervical spinal motion at multiple levels using an established in vitro cadaveric spine model. The two-level prostheses condition was also compared to the intact and two-level fused spinal condition.

\subsection{Materials and Methods}

\subsubsection{Specimen Preparation and Spinal Conditions}

Fourteen fresh human cadaveric sub-axial cervical spines (average age 63.2 \pm 5.9 years) were harvested, wrapped in saline soaked gauze, immediately placed in double plastic bags, and frozen at $-20^{\circ} \mathrm{C}$ until pre-testing preparation took place. Prior to preparation, specimens were thawed in a standard refrigeration system for 12 hours. Anterior-posterior and lateral radiographs were used to determine study inclusion. Exclusion criteria included gross osteopenia, anatomic abnormality, or unhealthy disc height. 
To prepare for testing, all vertebral bodies above the second cervical vertebral body (C2) and below the first thoracic vertebral body (T1) were removed. Excess musculature was removed with care taken to preserve spinal ligaments, discs, and bone. Boney surfaces were exposed at $\mathrm{C} 2$ and $\mathrm{T} 1$ to allow for potting material purchase. Screws were placed at the spinal processes, exposed facet joints, and vertebral bodies of $\mathrm{C} 2$ and $\mathrm{T} 1$ for added fixation of the potting material. Specimens were held upright with a positioning jig to maintain a neutral position. Positioning screws passing through the sides of the mold initially held the end bodies in place, while a low-melting-point bismuth alloy (Small Parts, Miami Lakes, FL) provided final fixation at the end bodies. Threaded rods were inserted into the posterior process enabling motion tracking target attachment and external fusion fixation. These rods did not hinder device installation.

Testing conditions included: non-instrumented condition (harvested); instrumented with two-level fusion (fusion) at levels C5-C6 and C6-C7; and instrumented with two prosthetic devices at levels C5-C6 and C6-C7. The Prestige Low-Profile (LP) (Medtronic Sofamor Danek, Memphis, TN) or the ProDisc-C (Synthes Spine, West Chester, PA) cervical disc replacement devices were used for the prosthetic device condition. The Prestige-LP device contains a titanium ceramic composite fashioned in a ball and trough mechanism, and designed with a keel system for initial fixation. The ProDisc-C device includes two forged cobalt-chrome alloy endplates with an ultra-high molecular weight polyethylene insert on the interior plate. The disc devices are shown in Table 5-1. Fusion was simulated with a customized external fixation system consisting of AO clamps and rods (Synthes, West Chester, PA) similar to a system used by orthopedic surgeons. Six specimens were implanted with the Prestige-LP device at levels C5C6 
Table 5-1: Arthroplasty Devices. (Images used with permission by Medtronic Sofamor Danek and Synthes Spine.)

Device*
Image

*CAUTION: These devices limited by Federal Law (US) to investigational use. 
and C6-C7 and eight specimens were implanted with the ProDisc-C at these same levels. All specimens were fused using similar instrumentation. Specimen testing conditions are shown in Figure 5-1.

\subsubsection{Non-Destructive Biomechanical Testing}

All biomechanical testing was preformed at the Joint Implant Biomechanics Laboratory (JIBL) in the Biomedical Engineering Department at the University of Tennessee Health Science Center, Memphis. The JIBL displacement control biomechanical testing protocol has been previously described $^{8,13}$ and has been utilized in other cervical spine studies with fusion techniques $^{12,17,40}$ as well as other prosthetic disc devices. ${ }^{12,14}$ Displacement control loading more closely replicates in vivo conditions, ${ }^{13}$ and thus is favored over pure moment loading methods.

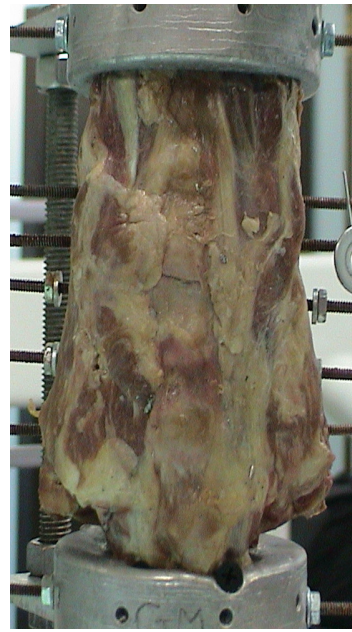

a

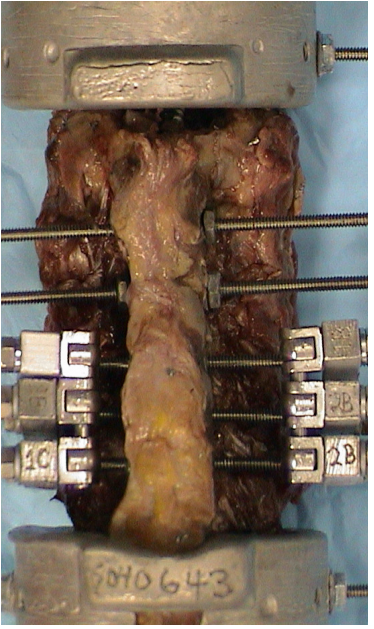

b

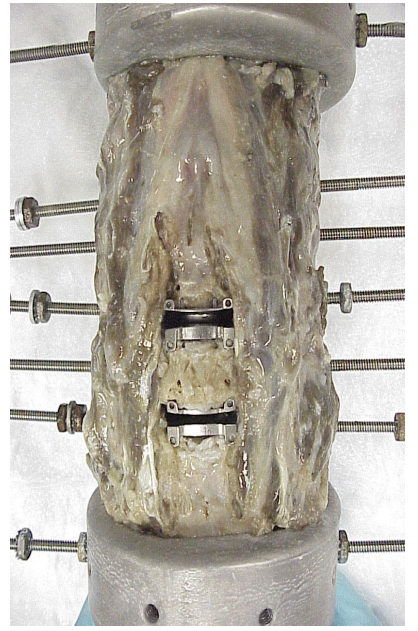

c

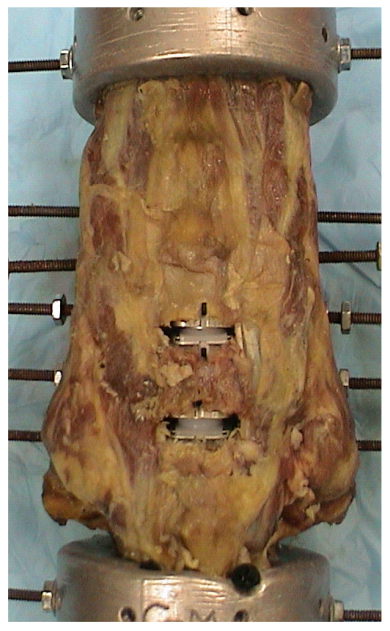

d

Figure 5-1: Spinal Conditions. (a) harvested, (b) two-level fusion, (c) two-level arthroplasty with Prestige-LP disc prosthesis, and (d) two-level disc arthroplasty with ProDisc-C prosthesis. 
In flexion, extension, and lateral bending conditions, specimens were compressively loaded at a $200 \mathrm{~mm}$ offset using a constant velocity of $6.4 \mathrm{~mm} / \mathrm{second}$ or approximately 2.0 $\mathrm{deg} / \mathrm{sec}$, until either a global moment of $3.5 \mathrm{Nm}$ or a global rotation of 45 degrees was reached. The specimen was mounted in a neutral inverted orientation, which applied a compressive load to the spine that induced a greater moment at $\mathrm{T} 1$ than $\mathrm{C} 2$; a physiologically favorable condition. ${ }^{12}$ Axial rotation end limits included a global moment of $3.5 \mathrm{Nm}$ or a global rotation of 45 degrees and a loading rate of $2.25 \mathrm{deg} / \mathrm{sec}$. Due to the motion coupling of lateral bending and axial rotation, ${ }^{37,48}$ specimens were permitted freely to rotate axially during lateral bending and to laterally bend unconstrained during axial rotation. ${ }^{12}$ All specimens were pre-conditioned with at least five cycles prior to the final trial of three consecutive cycles. Data analysis was carried out on the third cycle at a global moment limit of $3 \mathrm{Nm}$. Specimens were regularly moistened with a $0.9 \%$ saline mist throughout preparation and testing.

\subsubsection{Data Management and Statistics}

Global rotation, applied load, global moment, and individual relative MSU rotations were measured. Normalized flexibility, normalized motion, and change in MSU percent contribution values were calculated. Normalization was defined as either fused results or implanted results divided by respective harvested $(\mathrm{H})$ results for each MSU. Contribution of the implanted levels (C5-C7) relative to global contribution of the instrumented spine was normalized to the intact spine to determine motion response at the operated regions. Mean normalized values were calculated, grouped by operative condition (fusion, $n=14$; Prestige-LP, $n=6$; or ProDisc-C, $n=$ 8), and shown in bar graphs relative to the non-instrumented (harvested) result. Normalization compensates for tissue variability. Global moment values were uniform across the three different instrumented conditions for each loading case studied. 
Statistical analysis utilized the student's t-test to determine that the harvested and fusion condition of the two different device groups were not statistically different. Then one-way analysis of variance (ANOVA) was done to compare the four spine conditions. Significant differences detected by the ANOVA were further analyzed by Student Newman Keuls (SNK) test. Significance was defined as $\mathrm{P}<0.05$. For all groups, normality and equal variance was assumed.

\subsection{Results}

\subsubsection{Normalized Flexibility}

Normalized flexibility results are shown in Figures 5-2, 5-3, and 5-4. There were no significant differences between the Prestige-LP and ProDisc-C spine conditions in normalized flexibility except in right axial rotation $(121 \pm 24 \%$ of harvested contribution for Prestige-LP versus $145 \pm 36 \%$ for ProDisc-C). Fusion decreased flexibility in all cases, and was significantly different from the harvested condition in flexion $(76 \pm 8 \%$ of $\mathrm{H})$ and extension $(67 \pm 21 \%$ of $\mathrm{H})$. Fusion was significantly different from the Prestige-LP condition in flexion ( $76 \pm 8 \%$ versus 93 $\pm 17 \%)$, extension $(67 \pm 21 \%$ versus $152 \pm 43 \%)$ and right axial rotation $(95 \pm 12 \%$ versus $121 \pm$ 24\%). Fusion and ProDisc-C conditions were significantly different in flexion( $76 \pm 8 \%$ versus $97 \pm 17 \%)$, extension ( $67 \pm 21 \%$ versus $140 \pm 34 \%)$, left lateral bending ( $94 \pm 9 \%$ versus $120 \pm$ $43 \%)$, and right axial rotation ( $95 \pm 12 \%$ versus $145 \pm 36 \%)$. In extension and right axial rotation, the Prestige-LP condition increased normalized flexibility significantly from the harvested condition (152 $\pm 43 \%$ of $\mathrm{H}$ and $121 \pm 24 \%$ of $\mathrm{H}$ respectively). The normalized flexibility pf the ProDisc-C condition was significantly different from harvested in 


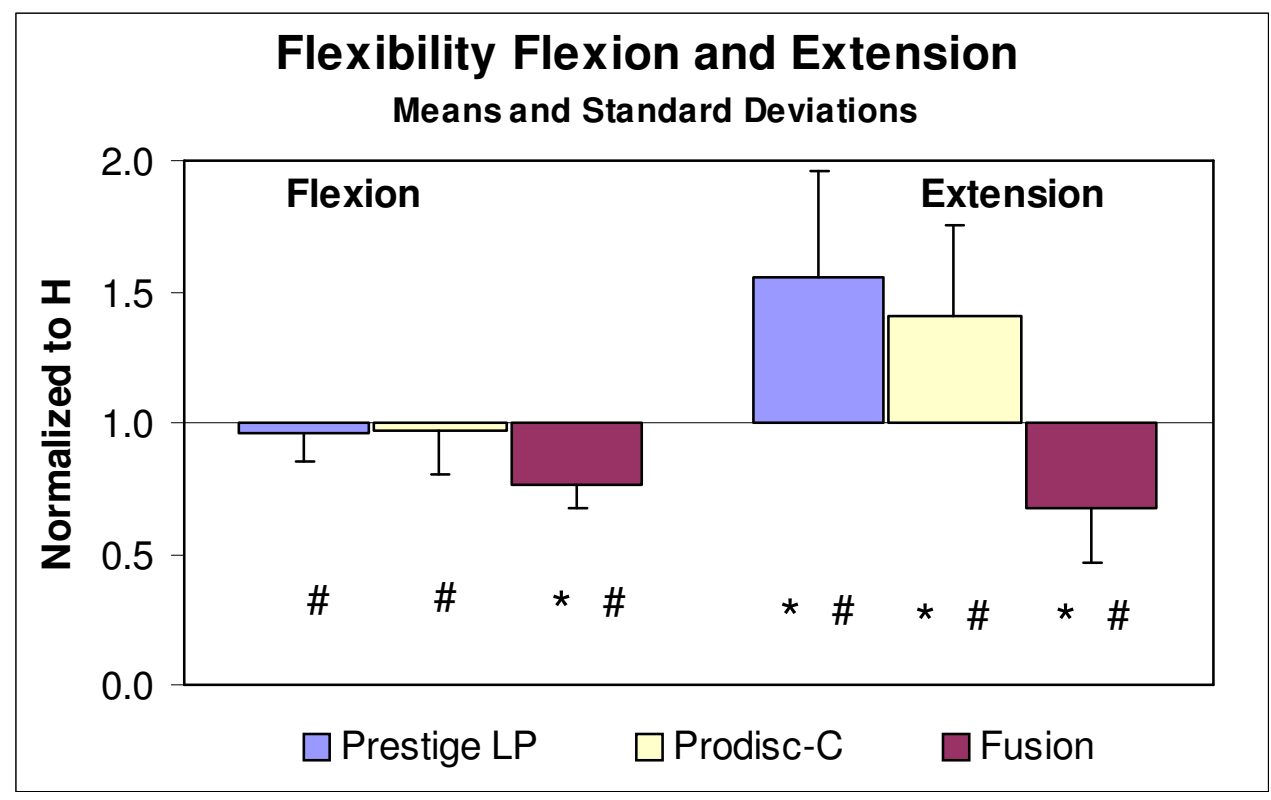

Figure 5-2: Normalized Flexibility in Flexion and Extension. All values relative to the harvested $(\mathrm{H})$ condition represented by unity. ${ }^{*}$ Indicates significant difference from harvested. \# Indicates significant difference between fusion and disc arthroplasty.

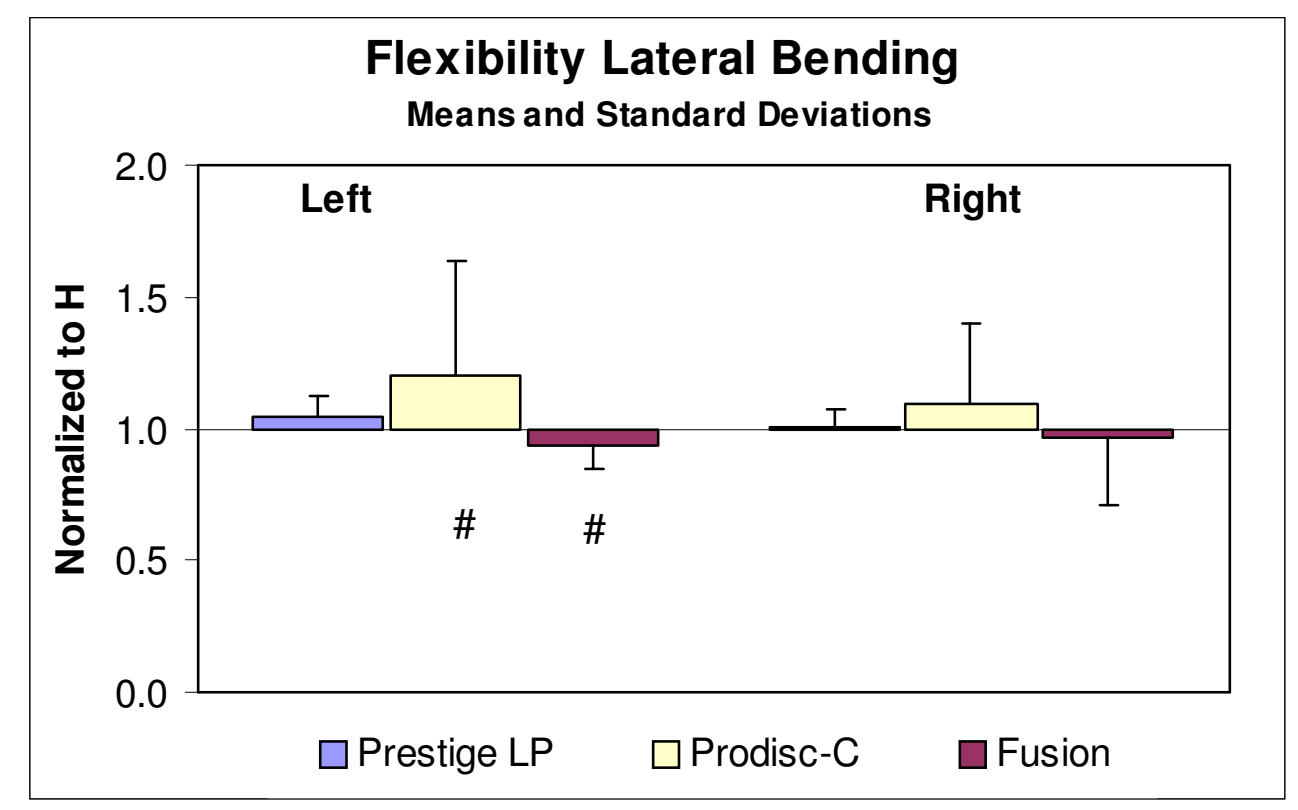

Figure 5-3: Normalized Flexibility in Lateral Bending. \# Signifies significant difference between fusion and disc arthroplasty. 


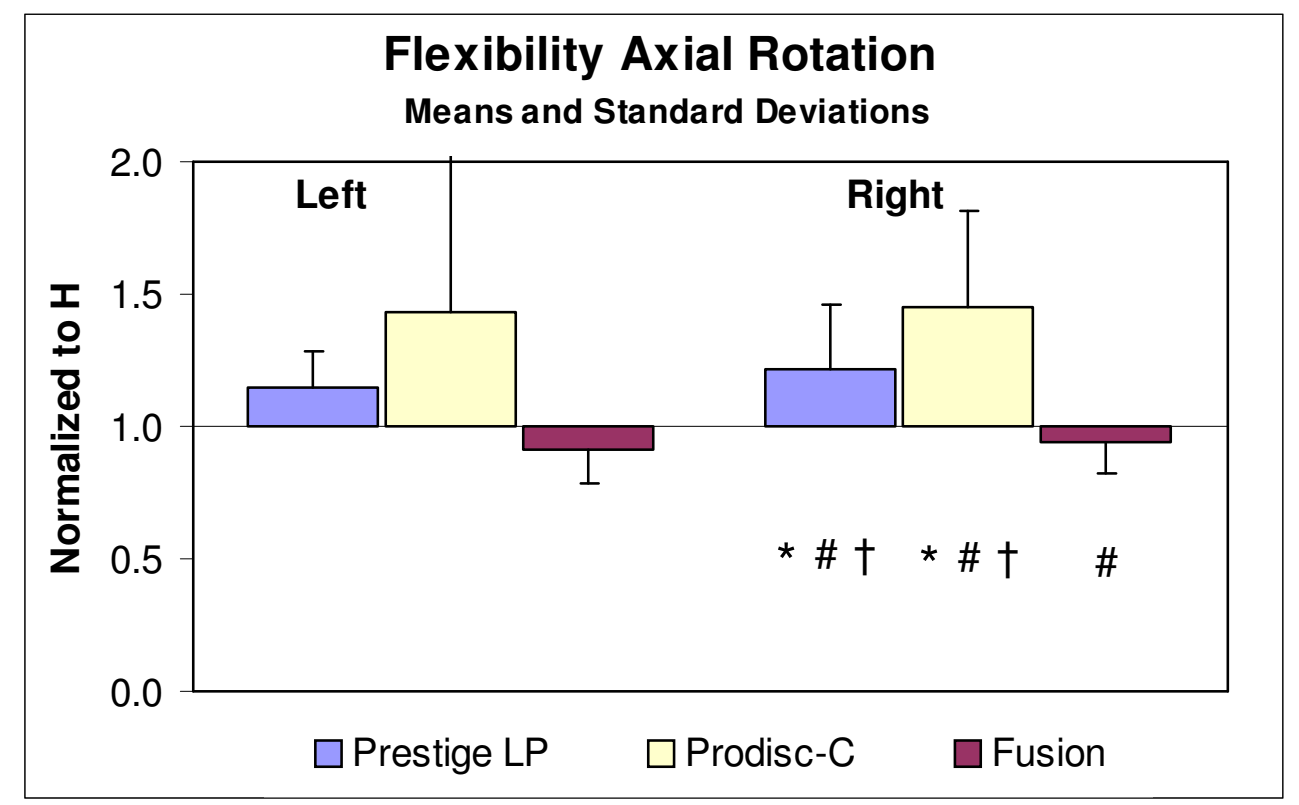

Figure 5-4: Normalized Flexibility in Axial Rotation. * Signifies significantly different from harvested. \# Signifies significant difference between fusion and disc arthroplasty. † Signifies significant difference occurred between the Prestige-LP and ProDisc-C.

extension, and right axial rotation (132 $\pm 27 \%$ and $134 \pm 17 \%$ of $\mathrm{H}$ respectively).

\subsubsection{Normalized Motion}

Instrumented motion was normalized to harvested and shown in Figures 5-5, 5-6, and 5-7. The only significant differences between the Prestige-LP and the ProDisc-C spine conditions were in extension $(126 \pm 21 \%$ vs. $99 \pm 38 \%)$, and combined left and right axial rotation $(105 \pm$ $8 \%$ vs. $136 \pm 51 \%$ ). The ProDisc-C condition was significantly different from harvested in flexion $(117 \pm 18 \%$ of $\mathrm{H})$, left axial rotation $(122 \pm 24 \%$ of $\mathrm{H})$, and combined left and right axial rotation $(123 \pm 37 \%$ of $\mathrm{H})$. Significant differences between Prestige-LP and harvested conditions included flexion (114 $\pm 14 \%$ of $\mathrm{H})$, extension $(126 \pm 21 \%$ of $\mathrm{H})$, and 


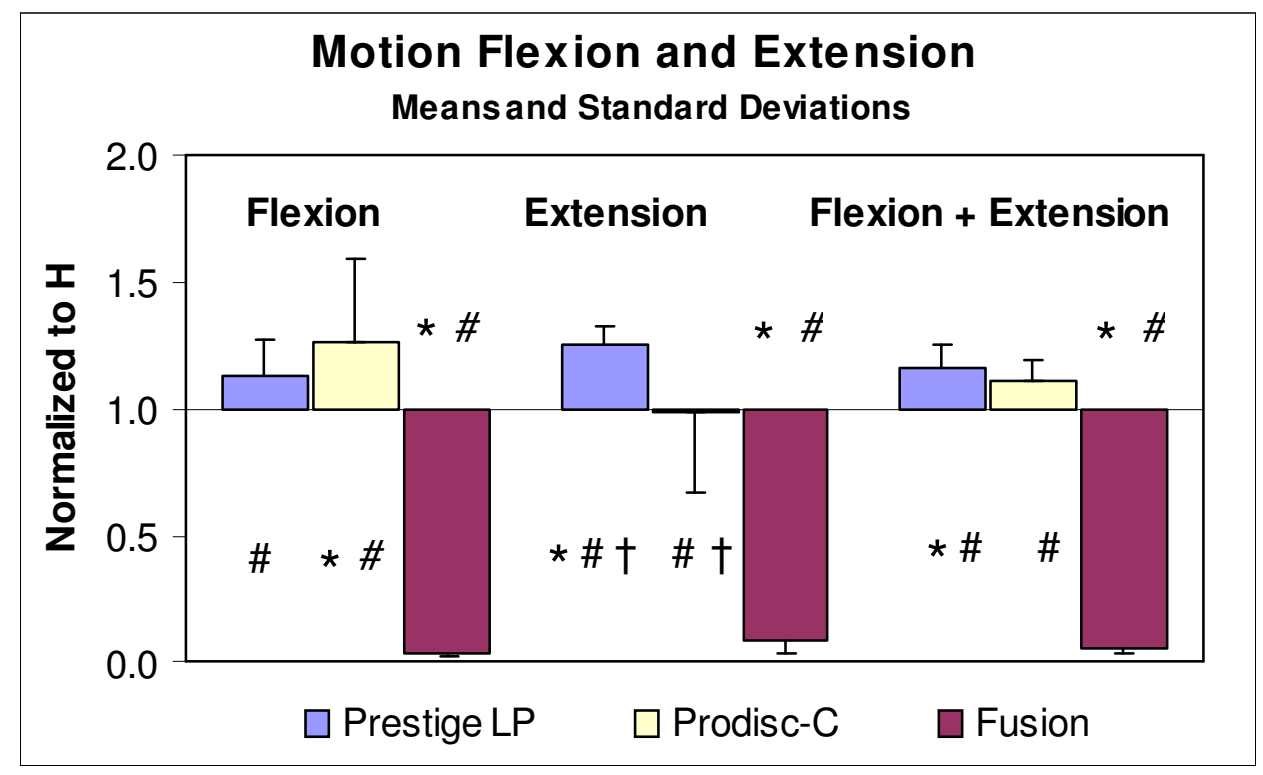

Figure 5-5: Normalized Motion in Flexion and Extension. $\dagger$ Signifies significant difference between the Prestige-LP and ProDisc-C conditions. * Signifies significant difference from the harvested $(\mathrm{H})$ condition. \# Signifies significant difference between fusion and Prestige-LP and ProDisc-C.

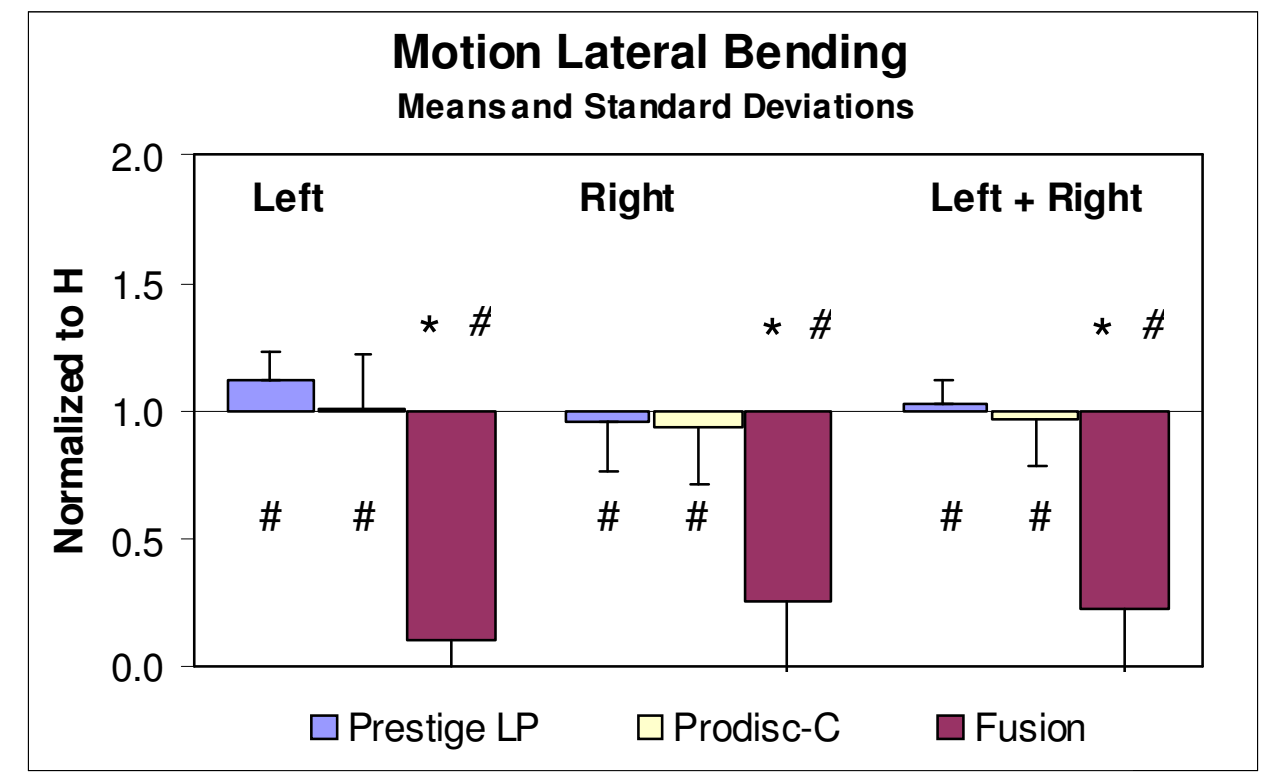

Figure 5-6: Normalized Motion in Lateral Bending. * Signifies significant difference relative to harvested $(\mathrm{H})$. \# Signifies significant difference between fusion and disc arthroplasty. 


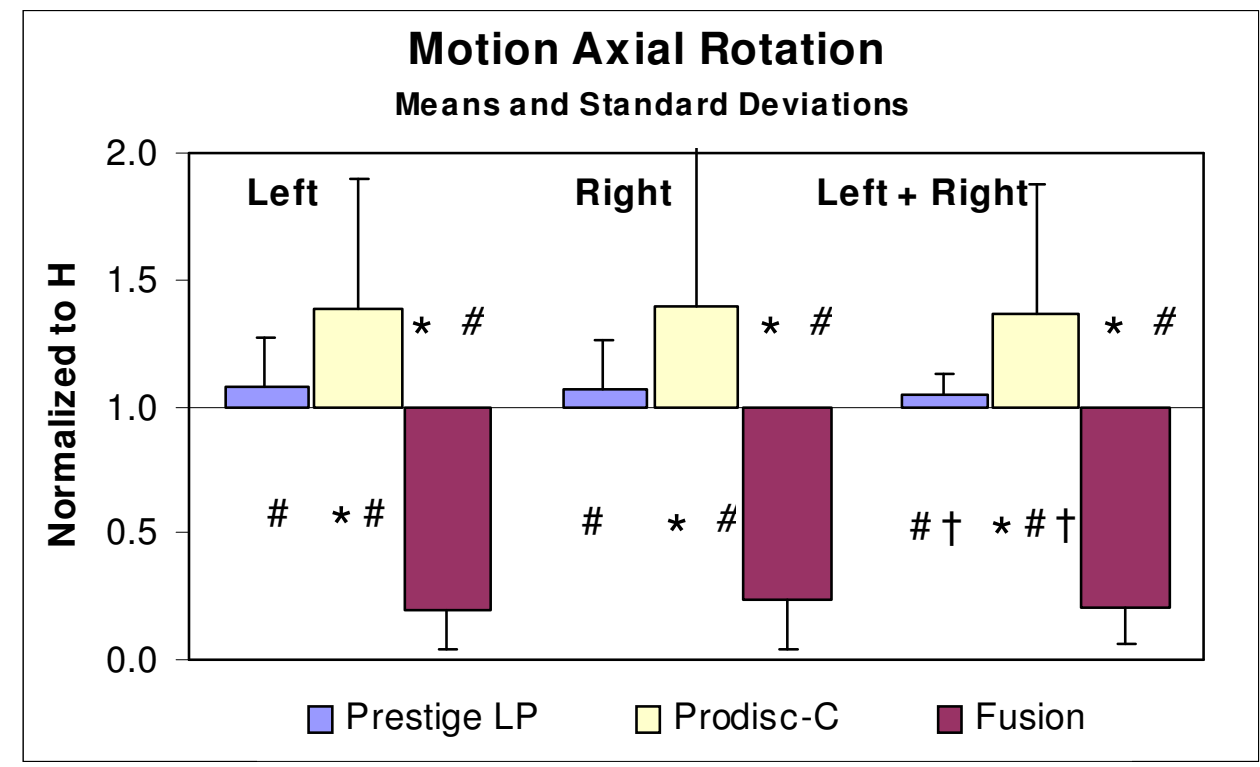

Figure 5-7: Normalized Motion in Axial Rotation. $†$ Signifies significant difference occurred between the Prestige-LP and ProDisc-C. * Signifies statistical difference from harvested (H) motion. \# Indicates a significant differences between fusion and disc arthroplasty.

combined flexion plus extension $(117 \pm 7 \%$ of $\mathrm{H})$. As expected, fusion decreased normalized motion in all loading conditions as well as combined flexion/extension, left/right lateral bending, and left/right axial rotation as compared to the harvested condition. A significant difference was found in all cases using one way ANOVA followed by SNK with $\mathrm{P}<0.05$. Fusion also significantly decreased normalized motion as compared to the Prestige-LP and ProDisc-C spine conditions for all loading cases.

\subsubsection{MSU Percent Change in Contribution}

The percent change in motion for all levels is shown in Figures 5-8, 5-9, and 5-10.

Within the operated region, the only significant difference between the Prestige-LP and ProDisc$\mathrm{C}$ spine conditions occurred at $\mathrm{C} 6-\mathrm{C} 7$ in extension. The ProDisc-C condition was 

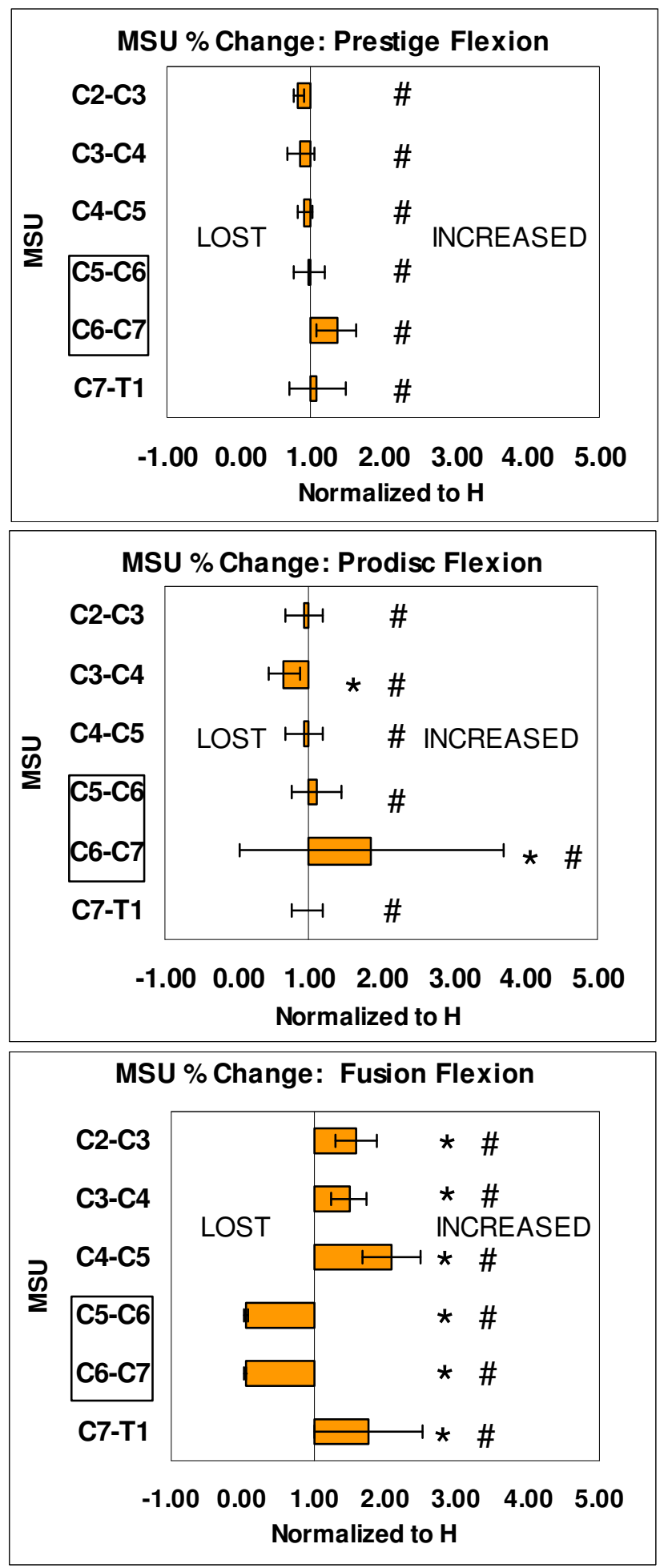

$\mathbf{a}$
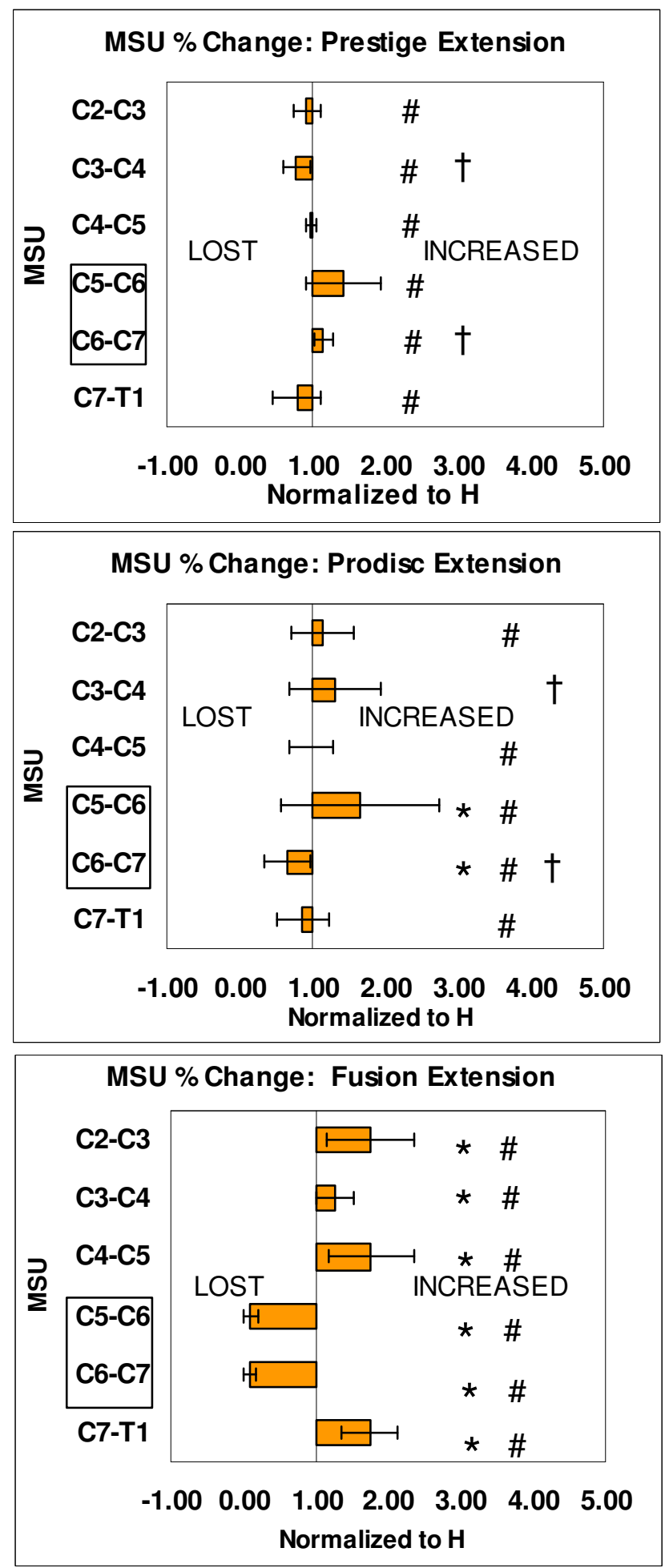

b

Figure 5-8: Percent Change Contribution for MSU Rotations. (a) flexion and (b) extension. * Statistical difference from harvested $(\mathrm{H})$. \# Signifies significant difference between fusion and disc arthroplasty. † Signifies significant difference between Prestige-LP and ProDisc-C. 


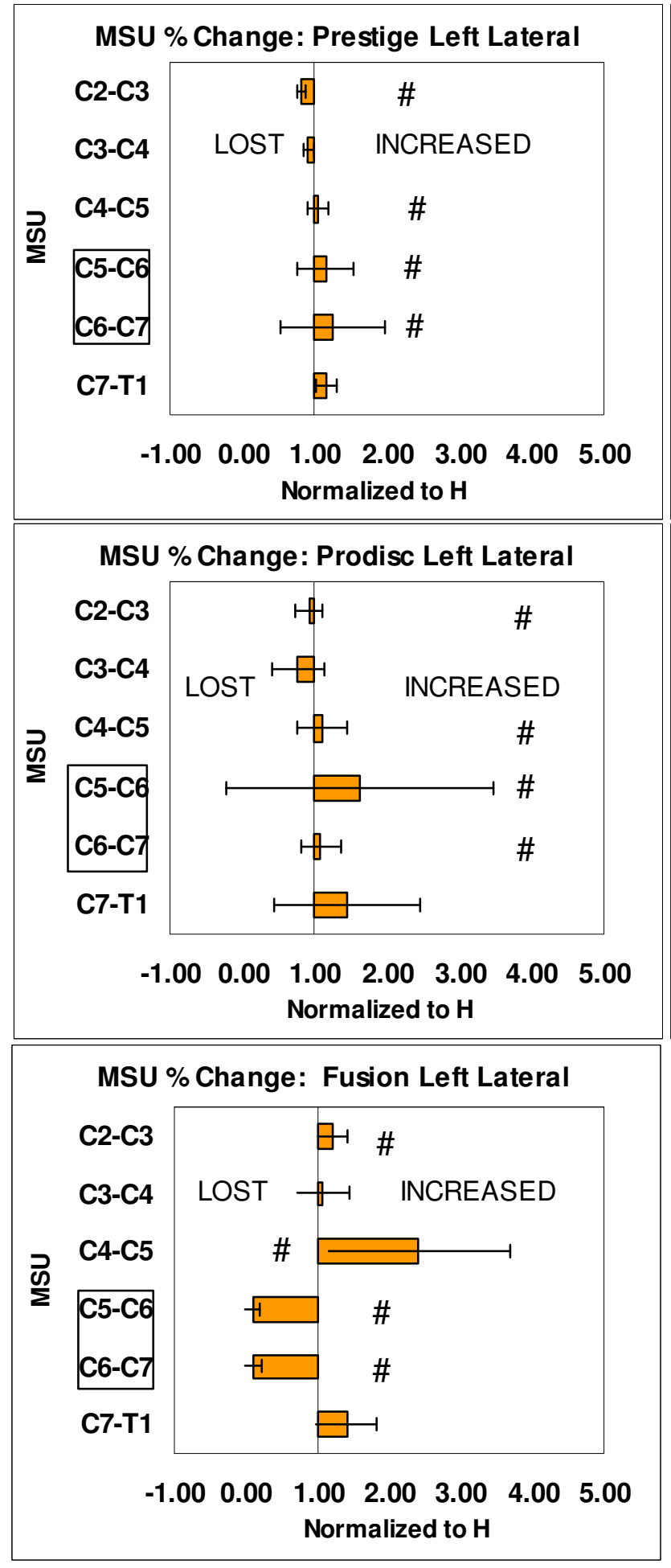

$\mathbf{a}$
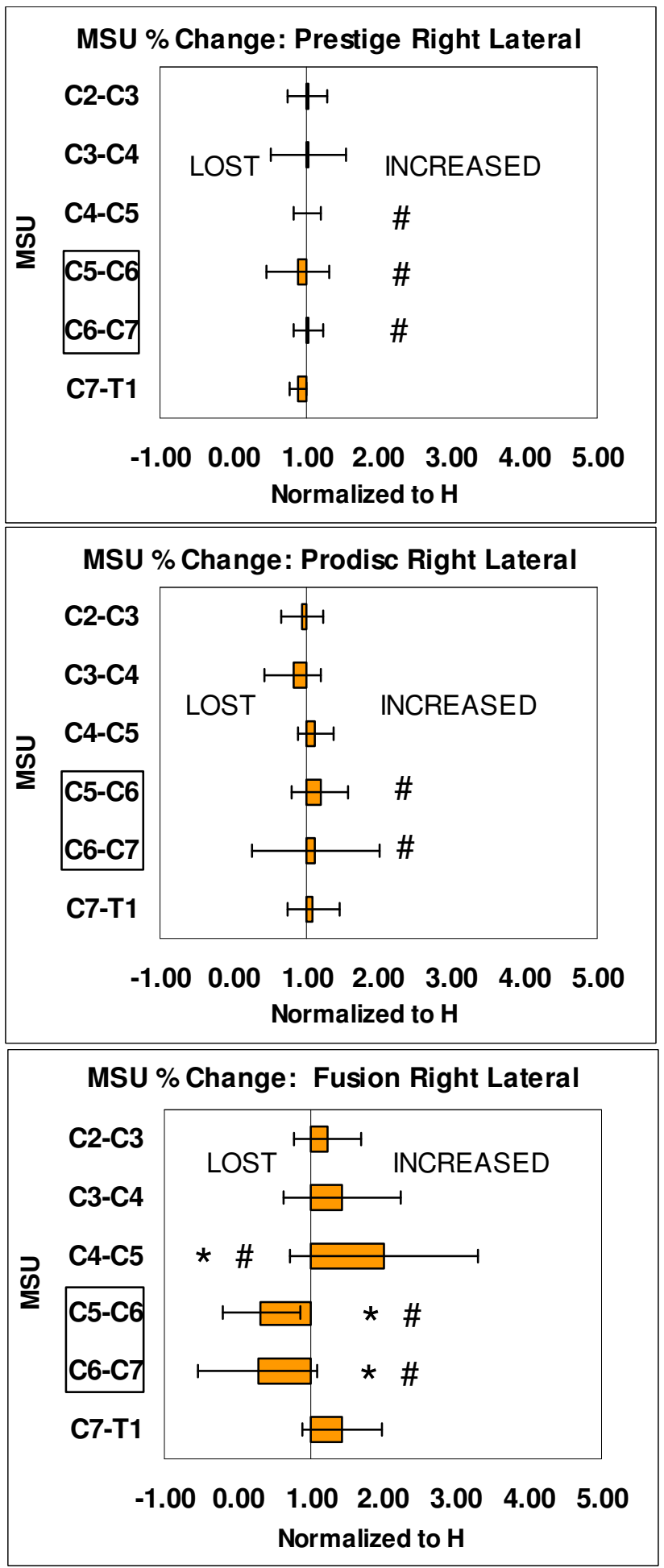

b

Figure 5-9: Lateral Bending Percent Change Contribution for MSU Rotations. (a) left and (b) right lateral bending. * Signifies significant difference from harvested $(\mathrm{H})$. \# Signifies significant difference between fusion and disc arthroplasty. 

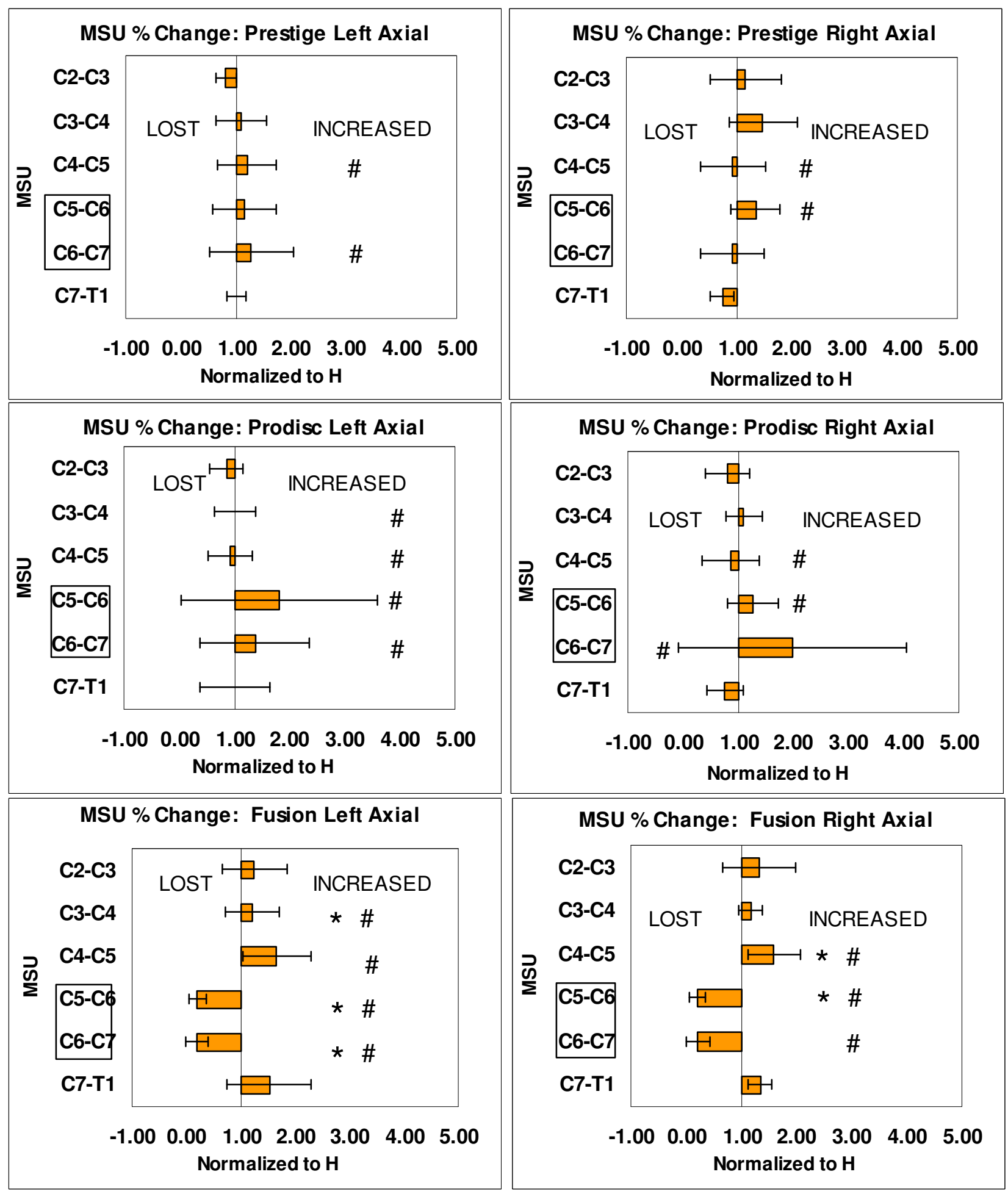

$\mathbf{a}$

b

Figure 5-10: Axial Rotation Percent Change Contribution for MSU Rotations. (a) left and (b) right axial rotation. * Signifies significant difference from harvested $(\mathrm{H})$. \# Signifies significant difference between fusion and disc arthroplasty. 
significantly different from the harvested condition at both operative levels in extension, however the motion at $\mathrm{C} 5-\mathrm{C} 6$ increased relative to the harvested condition decreased at C6-C7 relative to the harvested condition. A significant difference also occurred between ProDisc-C and harvested spine conditions at C6-C7 during flexion. A significant difference occurred between the Prestige-LP and fusion conditions in all testing modes at the operative levels except in left axial rotation at $\mathrm{C} 5-\mathrm{C} 6$ and right axial rotation at C6-C7. A significant difference occurred between the ProDisc-C and fusion spine conditions in all testing modes across both operative levels. Two-level fusion resulted in a significant decrease in motion at both C5-C6 and C6-C7 for all loading cases except during left lateral bending and left axial rotation at C6-C7 compared to harvested at the same levels.

For the levels directly inferior (C4-C5) and superior (C7-T1) to the operative levels, a statistically significant increase in motion occurred for fusion in all loading cases, except left lateral bending and left axial rotation at $\mathrm{C} 4-\mathrm{C} 5$, and combined left/right lateral bending and combined left/right axial rotation at C7-T1 compared to the harvested condition at the same level. There were no significant differences between Prestige-LP and harvested spine conditions for any loading case at these levels. The ProDisc-C spine condition had a significant decrease in motion relative to the harvested spine in flexion at $\mathrm{C} 3-\mathrm{C} 4$; otherwise no significant differences between ProDisc-C and harvested spine conditions occurred at the adjacent levels. Fusion had significantly increased motion compared to the Prestige-LP condition at C4-C5 in all loading conditions except right lateral bending. At C7-T1 there were only significant differences between Prestige-LP and fusion conditions during flexion and extension. The same pattern was true for ProDisc-C compared to fusion, except there was no significant difference at C4-C5 during right lateral bending. 


\subsection{Discussion}

Few clinical studies have presented two-level cervical arthroplasty. Goffin et al ${ }^{19}$ followed 26 Bryan Cervical Disc patients with two-level implantation for one year. At one year $96 \%$ of these patients reported excellent, good, or fair outcome. Of these, $86 \%$ of patients who received two adjacent level implants had a range of motion of $2^{\circ}$ or greater. Sekhon et $\mathrm{al}^{42}$ used the Bryan Cervical Disc prosthesis to treat cervical spondylitic myelopathy with an 18 month follow-up. Four of eleven patients were implanted at two levels in this study, either at C4-C5 and C5-C6, or C5-C6 and C6-C7. Three of these patients reported an excellent outcome. Other clinical trials evaluating cervical disc prosthesis included patients with two-level implantation, but analysis was based on the total number of devices implanted rather than grouping patients based on multi-level or single level arthroplasty. Duggel et al. ${ }^{15}$ published range of motion studies using static and dynamic digital radiographs to quantify range of motion, which included four patients receiving two-level Bryan cervical disc implants. Lateral radiographs were collected in neutral, flexion, and extension positions pre-surgery and up to 24 months postsurgery. While two-level implantation subjects were not analyzed separately from single level subjects, range of motion (ROM) was not significantly different post-implantation, demonstrating that the Bryan prosthetic disc ROM is similar to native disc ROM.

$\mathrm{McAfee}^{28}$ introduced a comparison of single level and multi-level cervical disc arthroplasty using the PCM prostheses in Sao Paylo, Brazil. Single level implantation occurred at C3-4 and multi-levels included C3 through C6. Fifty-five single level implantations were presented with nine revisions and four at the adjacent level. Fifty-four multi-level implantations were presented with 43 two-level implants, seven three level, and four quadruple level implantations. Of these multi-level patients, seven were revisions, and four were adjacent to 
fusion site. McAfee ${ }^{28}$ suggested the success of fusion decreases as number of fusion levels increases, while an increase in number of levels with disc arthroplasty produces much better results.

In the current study, biomechanical testing has demonstrated two-level disc arthroplasty to be more similar to the harvested condition then the fused condition. Further, the two-level Prestige-LP disc arthroplasty was not found significantly different from two-level ProDisc-C arthroplasty except in a few cases. An increase in normalized motion occurred with the ProDisc$\mathrm{C}$ condition during axial rotation, which may be due to the decompression associated with implantation of the ProDisc-C device. Proper placement of the ProDisc-C requires adequate decompression so as to limit facet involvement during axial motion. In addition, greater motion occurred at the superior implant (C5-C6) during left axial rotation and the inferior implant (C6C7) during right axial rotation for the ProDisc-C condition. This finding may be due to the placement and alignment of the artificial disc in the spine. Since ProDisc-C device encourages decompression, whichever level has the device placed more midline would be more involved in axial rotation motion. In contrast, the Prestige-LP is a lower profiled device and retains facet joints involvement in axial rotation. In extension, the Prestige-LP had an increase in motion, while the more constrained ProDisc-C implant retained similar motion to the harvested spine. The Prestige-LP is a more mobile device due to the anterior-posterior translation, which may explain the differences in extension between the two devices.

For the MSU percent contribution, both the Prestige-LP and the ProDisc-C disc devices showed an increase in motion at the lower implanted level during flexion and an increase in motion at the upper implanted level during extension as shown in Figure 5-8. This phenomenon is not yet explained through the present study, but may be due to an increase in motion caused by 
the devices which allows the spine to shift more on one implant level then the other in each motion.

There are four major design differences between the Prestige-LP and ProDisc-C disc prosthesis: 1) the type of articulating surface, 2) the total number of degrees of freedom provided by each disc design, 3) the $2 \mathrm{~mm}$ AP translation available in the Prestige-LP prosthesis, and 4) the location of the center of rotation. As compared earlier in Table 5-1, the Prestige-LP has a metal on metal articulating surface, while the ProDisc-C articulating surfaces are metal on UHMWPE. These differences suggest the movement of each device will slightly differ. The ProDisc-C prosthesis provides three coupled rotational degrees of freedom using a concave upper component and UHMWPE ball lower component with no independent translation. This results in a fixed center of rotation on the lower vertebral body in reference to the disc space. The Prestige-LP disc allows up to four degrees of freedom: three rotations and $2 \mathrm{~mm}$ of translation in the anterior-posterior direction. Having some translation permits limited movement of the center of rotation located in the superior endplate of the implanted disc space. Despite slight differences in device design, the constrained ball and socket type joint functioned similar to the semi-constrained disc and provided rotational movement comparable to the harvested condition. These results are consistent with Puttlitz et al. ${ }^{38}$ who determined the ball and socket design replicated physiological motion in all three motion planes. Based on the MSU percent contribution from this study, the ProDisc-C prosthesis has a decrease in motion at the lower implanted level (C6-C7) during extension and a significant difference occurred between the two prosthetic devices at the upper implanted level (C5-C6). The ProDisc-C prosthesis could be indicated for patients with limited faced competency and in need of more motion control and 
stability, while Prestige-LP prosthesis could be considered for those patients with early stage disease with normal functioning facets.

\subsection{Conclusion}

While ACDF procedures for treatment of cervical spine disease have very successful initial outcomes, it has been repeatedly shown that incidences of recurring surgery occur in as many as one out of five patients long-term. ${ }^{25}$ Complications of this nature introduce a rationale for investigating new treatments for end stage cervical disease. Arthrodesis is a widely accepted practice used to treat knee and hip degeneration. It is only natural that a spinal counterpart be the next advancement in degenerative diseases of the spine. By using two cervical disc prostheses, this study further demonstrates two-level disc arthroplasty presented in a human cadaver model biomechanically favors natural cervical motion over fusion and may be an acceptable treatment option for early and end stage cervical disc disease. The efficacy of multi-level disc arthroplasty will be better understood as more long term clinical studies are followed up and reported. 


\section{Discussion}

Cervical spine fusion was reported as early as 1890 when Hadra first wired two vertebral bodies together with a posterior wiring technique. ${ }^{10}$ As spinal technologies develop, a wide variety of treatment options have become available for degenerative diseases of the cervical spine. Many techniques and instrumentation have focused on limiting motion and increasing stability of the cervical spine through a fusion procedure. ACDF procedures have been widely accepted since the 1950 's. ${ }^{25}$ Cervical plates, cages, grafts, etc. have all been used as fusion technologies with varied success. However, recent studies have reported ACDF causes long term complications that potentially lead to a second procedure in approximately one in five patients. $^{25}$ Other long term clinical follow-up studies report a repeat operation incidence of 6$7 \%$ for adjacent segment disease after ACDF. ${ }^{5,20}$ Symptomatic adjacent segment disease is the number one surfacing concern in the literature regarding limitations to fusion procedures, $3,9,20,23$, $24,27,38,39,44$ with the incidences increasing as more levels are fused. ${ }^{28}$ In fact, using a mathematical model Matsunaga et al. ${ }^{27}$ was able to estimate adjacent disc shear strain in two- or three-level ACDF procedures. The model showed an increase in shear strain of $20 \%$ at levels adjacent to multi-level fusion after one year, while similar results did not occur for single level fusion. Abnormal longitudinal strain was also observed. In discs with abnormal longitudinal strain, $73 \%$ occurred at segments adjacent to the fused segments. In a biomechanical study focusing on intradiscal pressure adjacent to a fused site, Eck et al. ${ }^{16}$ found a significant increase in intradiscal pressure adjacent to fusion at both levels during flexion, with a greater increase at C4-C5. Adjacent intradiscal pressure also increased in extension with a greater increase at C6C7, but the increase was not significant. As such, disc arthroplasty is a viable an alternative to 
fusion surgery that minimizes adjacent level disease, especially in multi-level cervical degenerative disease.

Some controversy exists as to whether the presence of adjacent segment disease following $\mathrm{ACDF}$ is due to the fusion surgery itself or the natural progression of degeneration with age. Never-the-less, in a long-term follow-up of a pediatric population treated with ACDF for severe spinal fracture and dislocation, an increased rate of adjacent segment disease occurred. ${ }^{29}$ This finding suggests that adjacent segment disease is influenced greatly by fusion of a disc space and decreased mobility at the operative level more so then degeneration with age. Other biomechanical studies have reported an increase in stress at the segments adjacent to a fused level that would enhance the progression of the disease. If this is truly the case, as the literature suggests, then an alternative to fusion in the motion preservation school of thought is a better objective.

Disc arthroplasty is an exciting possibility for the treatment of degenerative disc diseases of the cervical spine. By preserving motion at the effected level while removing the painful disc, arthroplasty provides an alternative option fusion cannot achieve. The lineage of the disc prosthesis design can be seen through the progression of initial attachment mechanisms changing from screws, which caused failure in early studies, to the keel positioning strategy $y^{26}$ used in the currently studied disc prosthesis to create a neater profile for imaging purposes and reduces the incidence of swallowing problems. By testing these devices in a two-level implanted study, it was shown that the initial immediate attachment was effective. The value of this research is critical as more clinical studies considering two-level disc arthroplasty for treatment of two-level degenerative disc diseases. Clinical cases with three or four disc arthroplasty have also been reported. ${ }^{39}$ If disc arthroplasty is to complement fusion as a future FDA approved option, it is 
important to understand the biomechanical effects of a multi-level implanted system, and a twolevel model is a reasonable place to begin.

\subsection{Summary of Two-Level Prestige-LP Versus Two-Level Fusion}

Two-level disc arthroplasty with the Prestige-LP cervical disc prosthesis maintained motion of those levels in an in vitro cadaveric model. A significant increase in motion of the two-level Prestige-LP spine condition occurred relative to the harvested condition in a few cases such as normalized flexibility data in combined flexion and extension, MSU percent contribution data at the C5-C6 level in extension, and the MSU percent contribution data at the C6-C7 level in extension, flexion, combined flexion and extension, and right lateral bending. The Prestige-LP was statistically similar to the harvested condition in all other loading modes. Fusion, on the other hand, was statistically different from the harvested condition. In MSU percent contribution in flexion, the lower implanted level (C6-C7) experienced a significant increase in motion, while in extension the upper implanted level (C5-C6) underwent significantly more motion. A biomechanical rational for this event does not exist, yet may be due to increases in motion at the instrumented level and/or a change in the instantaneous axis of rotation of the instrumented C5C7 complex. Further research is necessary to completely explain this motion behavior. Overall, fusion was significantly different from the Prestige-LP and harvested condition, but minimal differences occurred between the Prestige-LP and the harvested conditions. 


\subsection{Summary of Two-Level ProDisc-C Versus Two-Level Fusion}

The biomechanical response of two-level disc arthroplasty with the ProDisc-C implant was similar to the harvested condition except for a significant increase in normalized flexibility in extension and right axial rotation, normalized motion in flexion and left axial rotation, and MSU percent contribution in flexion and extension at C6-C7. At cervical spine levels inferior and superior to the treated levels, two-level ProDisc-C arthroplasty maintained physiological motion, while two-level fusion experienced a significant increase in motion at these levels. Consequently, motion at the treated levels and adjacent segments remained physiological with the ProDisc-C implant. The current study supports clinical findings that two-level disc arthroplasty provides a more favorable outcome then two-level fusion when motion preservation and adjacent segment disease issues are considered.

\subsection{Summary of Two-Level Prestige-LP Versus Two-Level ProDisc-C}

This study shows the similarities between devices with the ball and trough mechanism. The ProDisc-C and Prestige-LP disc prostheses provide three rotational degrees of freedom, but the rotation axes of the ball-shaped surface are located on opposite endplates. The Prestige-LP disc also provides some anterior posterior translation, while the ProDisc-C implant does not. Despite these differences the two devices preformed similarly in all biomechanical tests. The main biomechanical difference was more motion occurred with the Prestige-LP discs in extension, which is expected considering its AP translation capabilities. The ProDisc-C discs has a fixed center of rotation but encourages full decompression of the spine when placed 
properly. This design approach limits facet joint involvement and may explain the differences in axial rotation with the ProDisc-C implants.

Lastly the MSU percent contribution data consistently showed an increase in motion at the inferior treated level in flexion, while an increase in motion was observed at the superior implanted level in extension for both the Prestige-LP and the ProDisc-C disc prostheses

\subsection{Limitations}

Limitations of this study are twofold: limitations associated with the biomechanical testing methodolgy, and limitations with the physical design constraints of the disc arthroplasty devices. In vitro biomechanical testing does not take into consideration the musculature of the cervical spine. In vivo muscle involvement influences the motion of the spine and the supporting neck muscles would likely increase spine stability. Additionally the testing method used herein only evaluates the motion response of the spine to non-physiologic loads (i.e. no muscle involvement). Never-the-less testing method used in this study was developed to closely replicate in vivo motion of the cervical spine. This approach is superior to pure moment driven biomechanical testing methods and those utilizing follower loads for evaluating disc arthroplasty and motion preservation devices. ${ }^{12}$

The camera tracking system is another limitation of biomechanical testing especially in axial testing. This particular camera system is accurate in its $x-y-p l a n e ~(25$ micron resolution), but is not as accurate in depth or the z-direction (50 to 100 micron resolution). Axial testing relies on the camera's ability to see depth of the target motion. However, since the conclusion of this study, the camera system has been modified so the cameras point downward to utilize their $\mathrm{x}-\mathrm{y}-\mathrm{plane}$ resolution for axial tests, and improve the rotational measurements. 
While initial results of clinical studies support disc arthroplasty in both the single- and multi-level case, limitations still exist and caution must be taken when considering any new surgical treatment or device. Surgical technique and experience have a great influence on the success of motion preservation devices. Complications can arise when too much bone is excised during a discectomy, as was seen in a single level pilot study of the Frenchy cervical joint. In the Frenchy study, excessive load transfer to the facet joints eventually required the removal of the device and subsequent fusion of the joint. ${ }^{49}$ Another case showed significant vertebral body milling leaving only two to three millimeters of vertebral body between the two devices. In this patient significant milling was required due to excessively small vertebral bodies, however, this did not produce severe complications. ${ }^{35}$ Sizing of the prosthesis can also limit success. Both the Prestige-LP and ProDisc-C offer a variety of sizes and proper sizing is a key component in disc arthroplasty success. Cases of undersized prosthesis have caused severe postoperative pain and limited motion. ${ }^{34}$

For at patient with some initial onset of load instability, a more constrained device may be preferred to control the end ROM. Pickett et al. ${ }^{34}$ reported ROM of $11^{\circ}$ in flexion and extension with the Bryan cervical disc. The use of soft tissue retention in patients with large preoperative ROM may not be suitable candidates for multi-level disc arthroplasty.

\subsection{Future Work}

Two, three, and even four level fusion, as well as two and three level disc arthroplasty has been reported in the literature. McAfee ${ }^{28}$ noted that as the number of fused levels increases, fusion success rate decreases. They suggested that increasing levels of disc arthroplasty seem to 
produce better results, although long term clinical evaluations have not yet been reported. As more consideration is give to treating multiple levels with disc arthroplasty, a need exists to provide biomechanical data on three and four level disc arthroplasty cases in the cadaveric cervical spine scenarios. Other clinical scenarios exist that should be supported with good biomechanical data. For example, comparing the effect single level versus two-level arthroplasty has on the biomechanical integrity of the spine helps understand if addition of a second disc prosthesis adversely compromises the spine and may further impact decisions on placing a disc prosthesis adjacent to an existing fusion.

One of the main differences between the design of the ProDisc-C and Prestige-LP discs is the location of the axis of rotation of the device. The ProDisc-C disc has a fixed axis of rotation located near the inferior vertebral body end plate, while the Prestige-LP disc reports a floating axis of rotation located near the superior vertebral body end plate and allows up to 2-mm AP translation. Despite these differences the two devices behaved similar in restoring function in a two level case; the MSU percent contribution in flexion and extension was comparable. Recall an increase in motion occurred at C6-C7 during flexion relative to the harvested spine condition, while an increase in motion occurred at C5-C6 during extension relative to the harvested condition. To further understand the biomechanics of this trend instantaneous axis of rotation (IAR) patterns may help explain this phenomenon.

\subsection{Conclusions}

The biomechanical findings of two-level (C5-C6 and C6-C7) cervical disc arthroplasty support the choice of motion preservation as a viable alternative to multi-level anterior cervical discectomy and fusion. Two-level disc arthroplasty functioned similar to the harvested condition 
by retaining motion at the operative levels without increasing motion at the adjacent levels. Biomechanical tests of two-level fusion further confirmed that limiting motion at two levels causes an increase in motion at the adjacent levels.

Despite slight design differences between a ball and socket versus a ball-and-trough mechanism, the ProDisc-C and Prestige-LP discs performed similarly. The slight AP translational component associated with the Prestige-LP disc increased motion as in extension, while the ProDisc-C increased motion in axial rotation. In conclusion, two-level disc arthroplasty retains the integrity of the cervical spine and may be a viable surgical option for cervical degenerative disc disease at two adjacent levels compared to fusion surgery. 


\section{List of References}


1. An HS, and Simpson JM, ed. Surgery of the Cervical Spine. London: Martin Dunitz, 1994.

2. Anderson PA and Rouleau JP. Intervertebral disc arthroplasty. Spine 2004;29:2779-86.

3. Azmi H and Schlenk R. Surgery for postarthrodesis adjacent-cervical segment degeneration. Neurosurg. Focus. 2003;15:E6.

4. Bertagnoli R, Yue JJ, Pfeiffer F et al. Early results after ProDisc-C cervical disc replacement. J. Neurosurg.: Spine 2005;2:403-10.

5. Bohlman HH, Emery SE, Goodfellow DB, and Jones PK. Robinson anterior cervical disectomy and arthrodesis for cervical radiculopathy. Long-term follow-up of one hundred and twenty-two patients. J. Bone Joint Surg. Am. 1999; 75-A: 1298-307.

6. Center for Devices and Radiological Health. www.fda.gov/cdrh/ March 2006.

7. Chartbook of trends in the health of Americans. Health. United States: NCHS 2005

8. Chen J. Development of a flexible biomechanical testing apparatus. MS Thesis. 1996. University of Tennessee, Memphis.

9. Cherubino P, Benazzo F, Borromeo U, and Perle S. Degenerative arthritis of the adjacent spinal joints following anterior cervical spinal fusion: clinicoradiologic and statistical correlations. Ital. J. Orthop. Traumatol. 1990;16:533-43.

10. Clark C, ed. The Cervical Spine. 3rd ed. Philadelphia: Lippincott-Raven, 1998.

11. Cummins B, Robertson J, and Gill S. Surgical experience with an implanted artificial cervical joint. J. Neurosurg. 1998;88:943-8. 
12. DiAngelo D and Foley K. An improved biomechanical testing protocol for evaluating spinal arthroplasty and motion preservation devices in a multilevel human cadaveric cervical model. Neurosurg. Focus. 2004;17:E4.

13. DiAngelo D, Foley K, Morrow B et al. In vitro biomechanics of cervical disc arthroplasty with the ProDisc-C total disc implant. Neurosurg. Focus. 2004;17:E7.

14. DiAngelo D, Robertson J, Metcalf N, McVay B, and Davis R. Biomechanical testing of an artificial cervical joint and an anterior cervical plate. J. Spinal. Disord. Tech. 2003;16:31423.

15. Duggal N, Pickett GE, Mitsis DK, and Keller JL. Early clinical and biomechanical results following cervical arthroplasty. Neurosurg. Focus. 2004;17:E9.

16. Eck JC, Humphreys SC, Lim T et al. Biomechanical study on the effect of cervical spine fusion on adjacent-level intradiscal pressure and segmental motion. Spine 2002;27:2431-4.

17. Foley KT, DiAngelo DJ, Rampersaud YR, Vossel KA, and Jansen TH. The in vitro effects of instrumentation on multilevel cervical strut-graft mechanics. Spine 1999;24:2366-76.

18. Goffin J, Gensens E, Vantomme $\mathrm{N}$ et al. Long-term follow-up after interbody fusion of the cervical spine. J. Spinal Disord. Tech. 2004;17:79-85.

19. Goffin J, Van Calenbergh F, van Loon J et al. Intermediate follow-up after treatment of degenerative disc disease with the Bryan Cervical Disc Prosthesis: single-level and bi-level. Spine 2003;28:2673-8. 
20. Goffin J, van Loon J, Van Calenbergh F, and Plets C. Long-term results after anterior cervical fusion and osteosynthetic stabilization for fractures and/or dislocations of the cervical spine. J. Spinal Disord. 1995;8:500-8

21. Gore DR and Sepic SB. Anterior discectomy and fusion for painful cervical disc disease. A report of 50 patients with an average follow-up of 21 years. Spine 1998;23:2047-51.

22. Hilibrand AS, Carlson GD, Palumbo MA, Jones PK, and Bohlman HH. Radiculopathy and myelopathy at segments adjacent to the site of a previous anterior cervical arthrodesis. J. Bone Joint Surg. Am. 1999;81:519-28. 22.

23. JP Morgan, US Equity Research: Medical Supplies and Devices. Spine market update: thoughts and observations post-NASS. New York: JP Morgan, 2003.

24. Kulkarni V, Rajshekhar V, and Raghuram L. Accelerated spondylotic changes adjacent to the fused segment following central cervical corpectomy: magnetic resonance imaging study evidence. J. Neurosurg. 2004;100:2-6.

25. Le H, Thongtrangan I, and Kim DH. Historicial review of cervical arthroplasty. Neurosurg. Focus. 2004;17:E1.

26. Lee CK and Goel VK. Artificial disc prosthesis: design concepts and criteria. The Spine Journal 2004;4:209S-18S.

27. Matsunaga S, Kabayama S, Yamamoto T, Yone K, Sakou T, and Nakanishi K. Strain on intervertebral discs after anterior cervical decompression and fusion. Spine 1999;24:670-5. 
28. McAfee PC. Comparision of single level vs. multi-level cervical disc replacement - 178 consecutive PCM prostheses FDA pilot study - Sao Paulo, Brazil. Proceedings of the 2005 Spine Arthroplasty Society. New York, NY: 2005.

29. McGrory B and Klassen R. Arthrodesis of the cervical spine for fractures and dislocations in children and adolescents. A long-term follow-up study. J. Bone Joint Surg. Am. 1994;76:1606-16.

30. Merrill Lynch, US Medical Technology. NASS update: nothing compact about the artificial disc opportunity. New York: Merrill Lynch, 2003.

31. Park JB, Cho YS, and Riew KD. Development of adjacent-level ossification in patients with an anterior cervical plate. J. Bone Joint Surg. Am. 2005;87:558-63.

32. Phillips FM and Garfin SR. Cervical disc replacement. Spine 2005;30:S27-S33.

33. Pickett GE, Rouleau J.P., and Duggal N. Kinematic analysis of the cervical spine following implantation of an artificial cervical disc. Spine 2005;30:1949-54.

34. Pickett GE, Sekhon LH, Sears WR, and Duggal N. Complications with cervical arthroplasty. J. Neurosurg.: Spine 2006;4:98-105.

35. Porchet F. and Metcalf N.H. Clinical outcomes with the Prestige II cervical disc: preliminary results from a prospective randomized clinical trial. Neurosurg. Focus. 2004; 17:36-43.

36. Pospiech J, Stolke D, Wilke HJ, and Claes LE. Intradiscal pressure recordings in the cervical spine. Neurosurgery 1999;44:379-84. 
37. Puttlitz CM, Rousseau MA, Zu Z, Hu S, Tay BKB, Lotz JC. Intervertebral disc replacement maintains cervical spine kinetics. Spine 2004;29:2809-2814.

38. Roberston JT, Papadopoulos SM, and Traynelis VC. Assessment of adjacent-segment disease in patients treated with cervical fusion or arthroplasty: a prospective 2-year study. J. Neurosurg.: Spine 2005;3:417-23.

39. Sasso RC. Cervical kinematics: ACDF vs. artificial disc replacement. Proceedings of the 2005 Spine Arthroplasty Society. New York, NY: 2005.

34. Schwab J.S. Motion compensation associated with single-level cervicle fusion: a multilevel in-vitro study. MS Thesis. 2003. University of Tennessee Heath Science Center, Memphis.

41. Sekhon L. Cervicothoracic junction arthroplasty after previous fusion surgery for adjacent segment degeneration: case report. Neurosurgery 2005;56:E205.

42. Sekhon LH. Cervical arthroplasty in the management of spondylotic myelopathy: 18-month results. Neurosurg. Focus. 2004;17:E8.

43. Sekhon LH. Two-level artificial disc placement for spondylotic cervical myelopathy. J. Clin. Neurosci. 2004;11:412-5.

44. Sekhon LH and Ball JR. Artificial cervical disc replacement: principles, types and techniques. Neurology India 2005;53:445-4. 
45. Singh K, Vaccaro AR, Kim J, Lorenz EP, Lim TH, and An HS. Biomechanical comparison of cervical spine reconstructive techniques after a multilevel corpectomy of the cervical spine. Spine 2003;28:2352-8.

46. Smith HE, Wimberley DW, and Vaccaro AR. Cervical arthroplasty: material properties. Neurosurg. Focus. 2004;17:15-21.

47. White AA, Panjabi MM. Clinical Biomechanics of the Spine. 2nd ed. Philadelphia: J.B.Lippincott Company, 1990.

48. Wigfield C, Gill S, Nelson R, Langdon I, Metcalf N, and Robertson J. Influence of an artificial cervical joint compared with fusion on adjacent-level motion in the treatment of degenerative cervical disc disease. J. Neurosurg. 2002;96:17-21.

49. Wigfield C, Gill SS, Nelson RJ, Metcalf N.H., and Robertson J. The new Frenchay artificial cervical joint: results from a two-year pilot study. Spine 2002;27:2446-52. 


\section{Vita}

Laura Jan Gilmour was born in Wilmington, Delaware on November 24, 1981. She graduated with Honors from Oxford Area High School (Oxford, Pennsylvania), class of 2000. Laura went on to attend the University of Pittsburgh where she graduated Cum Laude with a Bachelor's of Science degree in Bioengineering. At the University of Pittsburgh she was student chapter president of Pitt's Society of Women Engineers, treasurer of the student chapter of Biomedical Engineering Society, and represented Pitt Biomedical Engineering at the BMES conference in Nashville, Tennessee. She studied abroad the summer of 2004 in the Pacific Rim with the Semester at Sea program before continuing her education in the Joint Biomedical Engineering program at the University of Tennessee Heath Science Center and University of Memphis in Memphis, Tennessee. 